\title{
Synthesis and therapeutic potential of imidazole containing compounds
}

\author{
Ankit Siwach and Prabhakar Kumar Verma*
}

\begin{abstract}
Imidazole is a five-membered heterocyclic moiety that possesses three carbon, two nitrogen, four hydrogen atoms, and two double bonds. It is also known as 1, 3-diazole. It contains two nitrogen atoms, in which one nitrogen bear a hydrogen atom, and the other is called pyrrole type nitrogen. The imidazole name was reported by Arthur Rudolf Hantzsch (1857-1935) in 1887. 1, 3-diazole is an amphoteric in nature i.e. it shows both acidic and basic properties. It is a white or colorless solid that is highly soluble in water and other polar solvents. Due to the presence of a positive charge on either of two nitrogen atom, it shows two equivalent tautomeric forms. Imidazole was first named glyoxaline because the first synthesis has been made by glyoxal and ammonia. It is the basic core of some natural products such as histidine, purine, histamine and DNA based structures, etc. Among the different heterocyclic compounds, imidazole is better known due to its broad range of chemical and biological properties. Imidazole has become an important synthon in the development of new drugs. The derivatives of 1, 3-diazole show different biological activities such as antibacterial, antimycobacterial, anti-inflammatory, antitumor, antidiabetic, anti-allergic, antipyretic, antiviral, antioxidant, anti-amoebic, antihelmintic, antifungal and ulcerogenic activities, etc. as reported in the literature. There are different examples of commercially available drugs in the market which contains 1, 3-diazole ring such as clemizole (antihistaminic agent), etonitazene (analgesic), enviroxime (antiviral), astemizole (antihistaminic agent), omeprazole, pantoprazole (antiulcer), thiabendazole (antihelmintic), nocodazole (antinematodal), metronidazole, nitroso-imidazole (bactericidal), megazol (trypanocidal), azathioprine (anti rheumatoid arthritis), dacarbazine (Hodgkin's disease), tinidazole, ornidazole (antiprotozoal and antibacterial), etc. This present review summarized some pharmacological activities and various kinds of synthetic routes for imidazole and their derived products.
\end{abstract}

Keywords: 1, 3-diazole, Antibacterial, Antitumor, Antioxidant, Antitubercular

\section{Background}

Nowadays, Public health problems were increasing due to AMR in drug therapy. So, there is necessary for the development of a new drug that overcomes the AMR problems [1].

In past, those drugs which contain heterocyclic nuclei give high chemotherapeutic values and act as a remedy for the development of novel drugs [2]. There are lots of heterocyclic compounds that are in clinical use to treat infectious diseases. So, there is a great importance of heterocyclic ring containing drugs [3].

*Correspondence: vermapk422@rediffmail.com

Department of Pharmaceutical Sciences, Maharshi Dayanand University, Rohtak, Haryana, India
In heterocyclic chemistry, imidazole containing moiety occupied a unique position [4]. It is a five-membered nitrogenous heterocyclic moiety that possesses three carbon, two nitrogen, four hydrogen atoms, and two double bonds having general molecular formula is $\mathrm{C}_{3} \mathrm{H}_{4} \mathrm{~N}_{2}$ (Fig. 1). The nitrogen atoms present at the first and third positions (non-adjacent position) of the ring [5], position four and five are equivalent [6]. It is also known as 1,3-diazole. It contains two nitrogen atoms, one nitrogen bear a hydrogen atom, and the other is called pyrrole type nitrogen [7]. 1,3-diazole ring is a bioester of the pyrazole ring [8]. It is the basic core of some natural products such as histidine, purine, histamine and DNA based structures, etc. [4]. The imidazole name was first reported by Arthur Rudolf Hantzsch (1857-1935) in 1887 [6]. 
1,3-diazole shows an amphoteric phenomenon i.e. it can behave like acid as well as a base. Two types of lone pair are present in the imidazole ring, delocalized and non-delocalized (non-Huckle) lone pair, i.e. both nitrogen of 1,3-diazole shows different dissociation constant. The dissociation constant (pKa) of delocalized lone pair and non-delocalized lone pair is 7 and 14.9 respectively. 1,3-diazole ring is susceptible to both electrophilic and nucleophilic attacks due to its amphoteric phenomenon [7]. For an acid imidazole, the dissociation constant is 14.5, which makes it less acidic than phenol, imides, and carboxylic acid except for alcohols (which is less acidic than imidazole). For a basic imidazole, the dissociation constant $(\mathrm{pKa})$ is approximately 7 (which makes imidazole 60 times more basic than pyridine). The acidic proton is present on the first nitrogen atom of the imidazole ring [6].

Due to the presence of a positive charge on either of the two nitrogen atoms, 1,3-diazole ring shows two equivalent tautomeric forms (Fig. 2) [9]. The presence of a sextet of $\pi$-electrons on the ring makes it an aromatic compound. The nitrogen atom on the third position in the imidazole ring is more reactive to the electrophilic compound due to the availability of unshared pairs of electron on the second nitrogen atom since the second nitrogen is a part of aromatic sextet [6].

It is a white or colorless solid. The imidazole ring shows excellent solubility in water and other polar solvents [10]. The dipole moment, melting point, and boiling point of the imidazole ring is $4.8 \mathrm{D}$ in dioxane [6], $88.9^{\circ} \mathrm{C}$, and $267.8^{\circ} \mathrm{C}$ [7] respectively. It possesses intramolecular hydrogen bonding [9].

Imidazole was first named glyoxaline because the first synthesis has been made by glyoxal and ammonia [9]. There is a different kind of synthetic route from which we can synthesize 1,3-diazloes, and its derivatives. Common methods are Debus-Radiszewski synthesis, Wallach synthesis, from dehydrogenation of imidazolines, from alpha halo-ketones, Marckwald synthesis, and amino nitrile [11].

Due to the polar nature of the imidazole ring, the pharmacokinetic parameters of the imidazole containing compounds should be improved to a great extent. Thus, this moiety helps to overcome the solubility problems of poorly soluble drug entities [12].

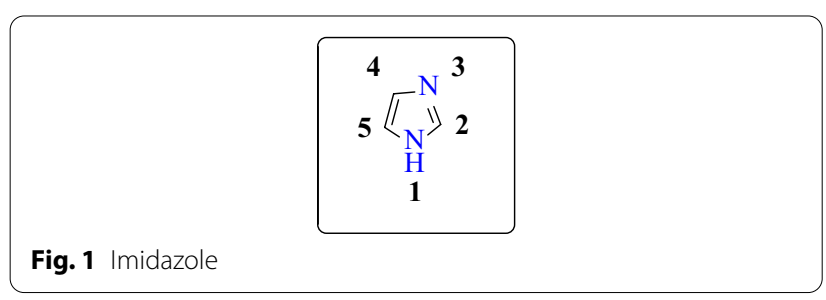

The 1,3-diazole and it's containing compounds shows a lot of therapeutic activities such as analgesics, antifungal, antihypertensive, antiobesity, antitumor [3], antiviral, anthelmintic, antitubercular [4], antiulcer, antihistaminic [13], anti-inflammatory, antidepressant [14]. antidiabetic [15], anticonvulsant [16], antiallergic [7], antirheumatic [17], antiasthmatic, alpha-blockers [18], antiprotozoal [19], antiaging, anticoagulant, antimalarial [20], and antiamoebic activity [21] etc.

There are different examples of commercially available drugs which consist 1,3,4-oxadiazole ring (Table 1) such as clemizole (antihistaminic agent), etonitazene (analgesic), enviroxime (antiviral), irtemazole, astemizole (antihistamine), omeprazole, pantoprazole (antiulcer), thiabendazole (antihelmintic), nocodazole (antinematodal) [22], metronidazole and nitrosoimidazole (bactericidal), megazol (trypanocidal) [12], azathioprine (anti-rheumatoid arthritis), tinidazole, ornidazole (antiprotozoal and antibacterial), satranidazole (amoebiasis), cimetidine (gastric ulcer), carbimazole (against thyroid disorder), tolazoline (vasodilator action), naphazoline (vasoconstrictor), tetrahydrozoline (vasoconstrictor) [16], etomidate, lansoprazole, flumazenil, methimazole, pilocarpine [19], ketoconazole [23], dacarbazine (anticancer) [24], pimobendan (calcium sensitizer and phosphodiesterase inhibitor) [25], fenbendazole [26].

\section{The mechanism for the formation of 2,4,5-trisubstituted imidazole}

The Debus-Radziszewski reaction mechanism for the formation of the 2,4,5-trisubstituted imidazole is given by (Scheme 1) [27].

\section{Main text}

\section{Antibacterial activity}

Jain et al. [28] synthesized 2-(4-substituted phenyl)1-substituted-4, 5-diphenyl-1H-imidazole (Scheme 2) and evaluated their antimicrobial activity against $S$. aureus, E. coli, and B. subtilis by cylinder wells diffusion method using Norfloxacin as a reference drug. Among the different derivatives, compounds $\mathbf{1 a}$ and $\mathbf{1 b}$ showed good antimicrobial potential. The conclusion of antibacterial activity was presented in (Table 2, Jain et al. [28]).

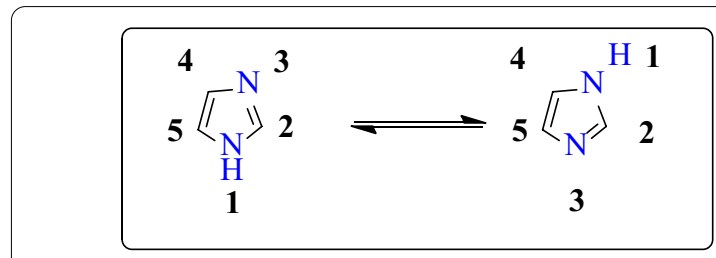

Fig. 2 Tautomeric forms of imidazole 
Table 1 Commercially available drugs are containing Imidazole nucleus

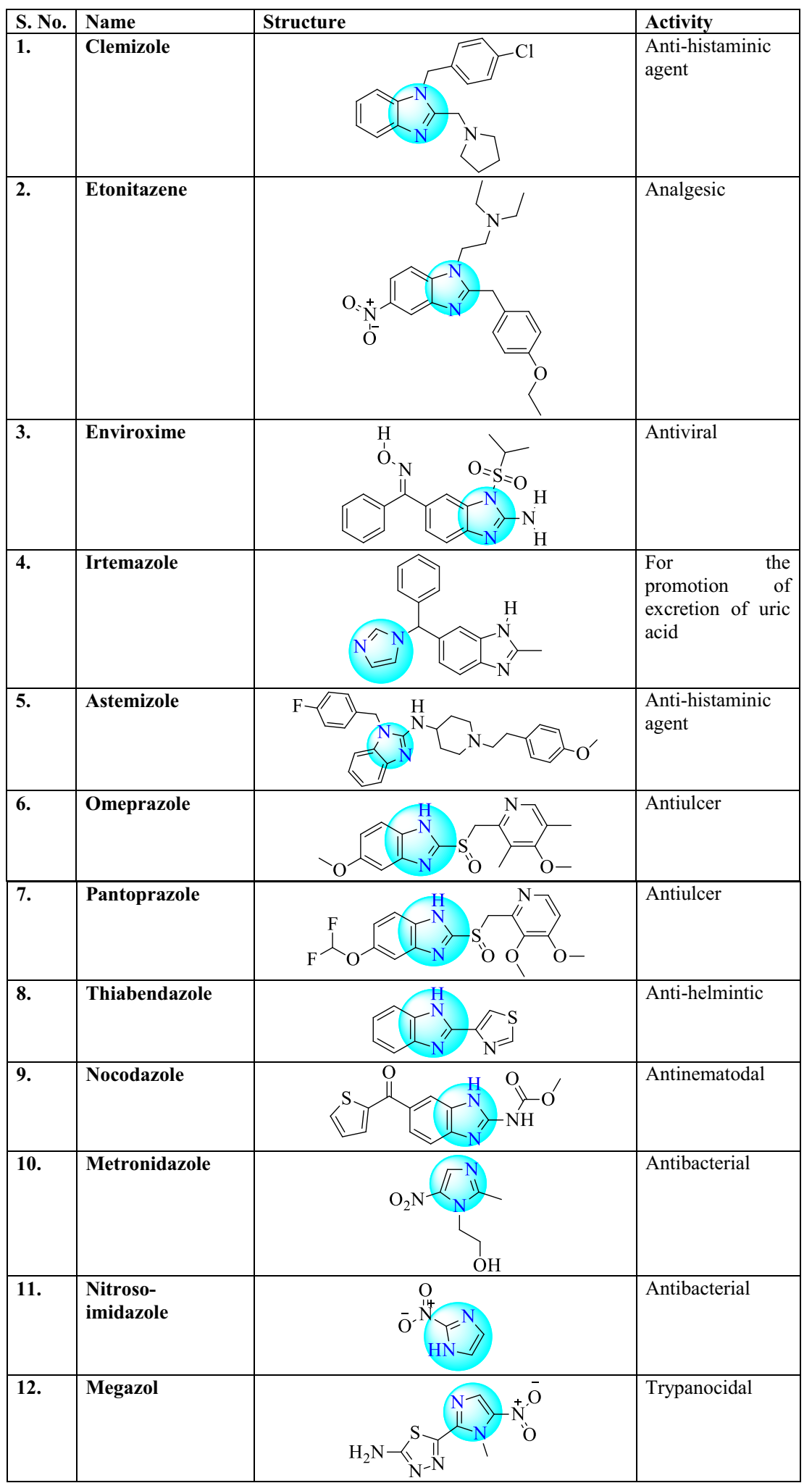


Table 1 (continued)

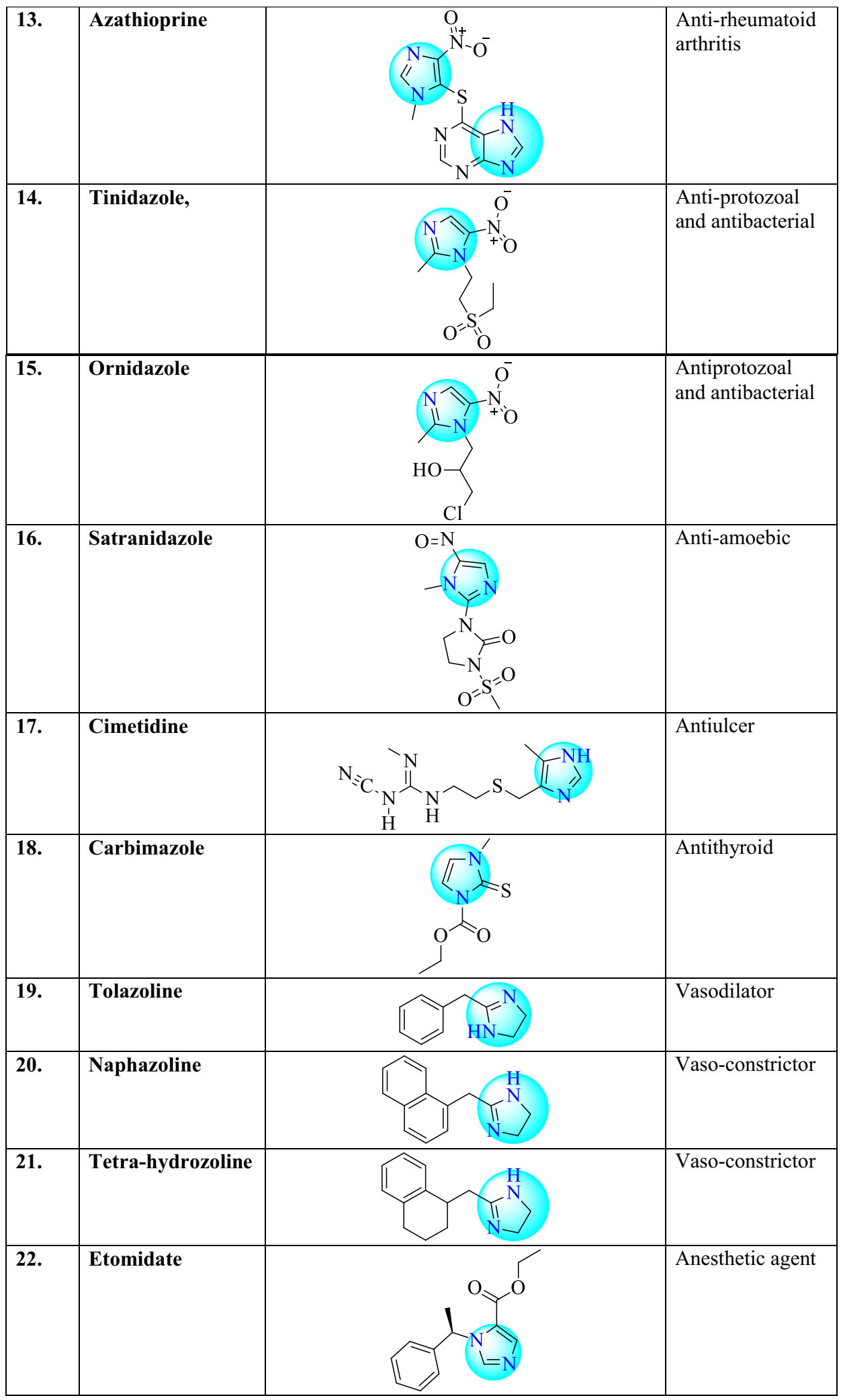


Table 1 (continued)

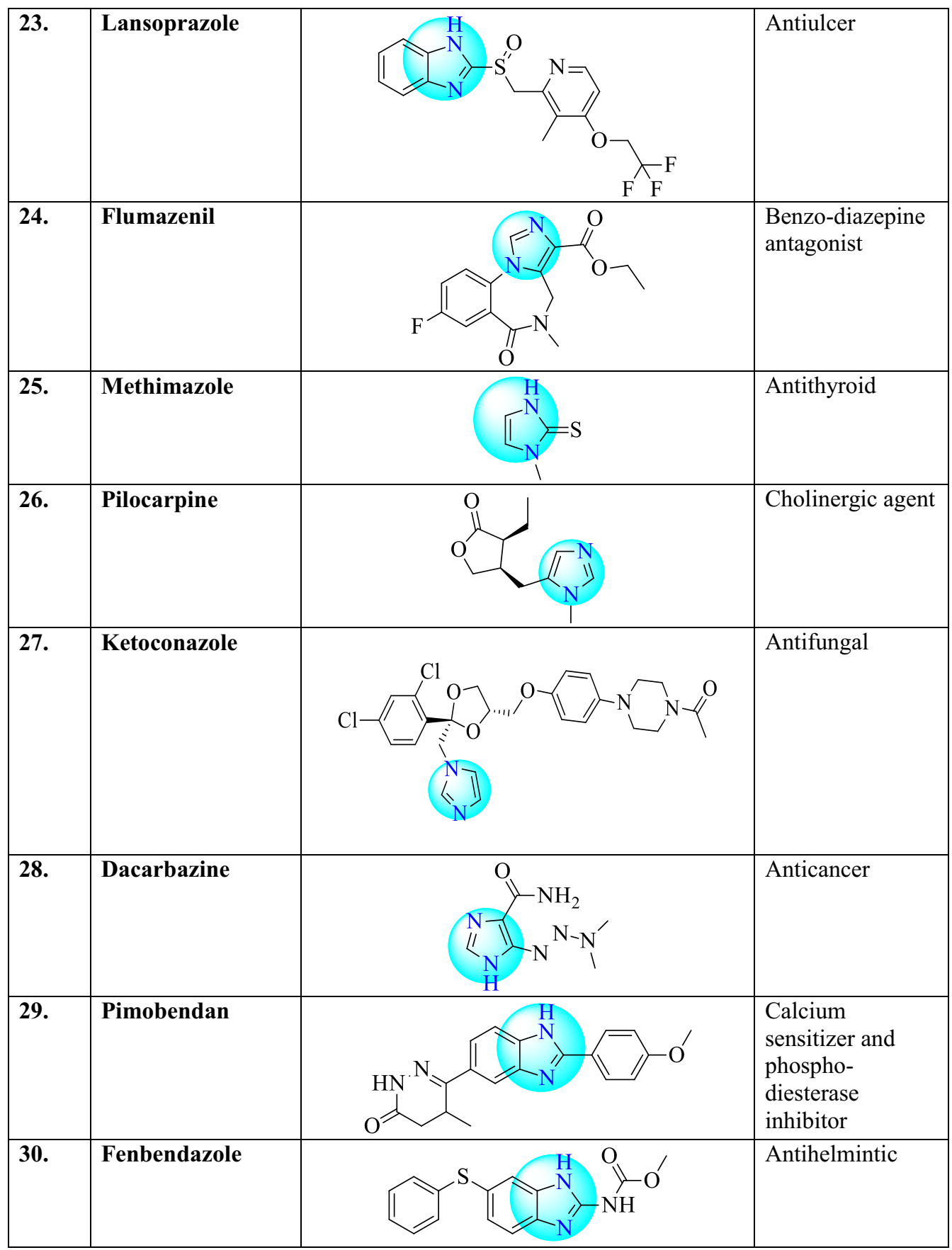

Narasimhan et al. [1] synthesized pyridin-3-yl (2-(2,3,4,5-tetra substituted phenyl)-1H-imidazol-1-yl) methanone (Scheme 3). The tube dilution method was used for the determination of antimicrobial potential against $S$. aureus, B. subtilis, and E. coli using ciprofloxacin as a reference drug. The antifungal activity of these derivatives was also evaluated against $A$. niger and $C$. albicans using Fluconazole as a reference standard. The conclusion of antimicrobial potential was presented in (Table 3, Narasimhan et al. [1]). 
Step 1.

$\mathrm{NH}_{4} \mathrm{OAc} \longrightarrow: \mathrm{NH}_{3}+\mathrm{AcOH}$

Step 2.

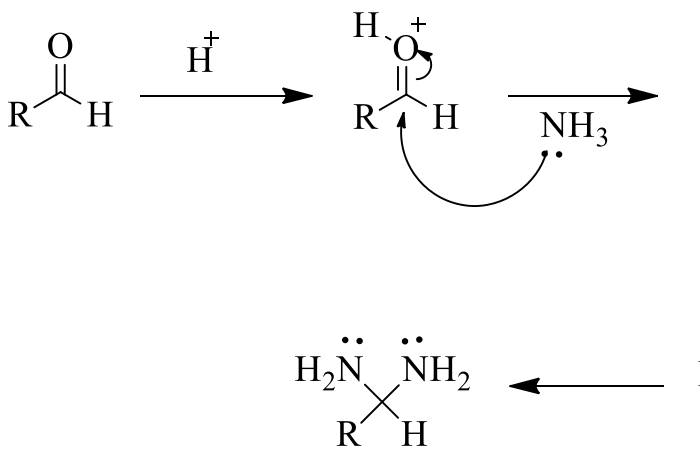

[A] .. $\mathrm{H}_{+} \mathrm{H}$

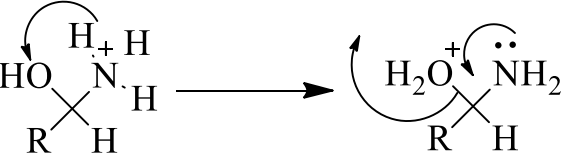

Step 3.

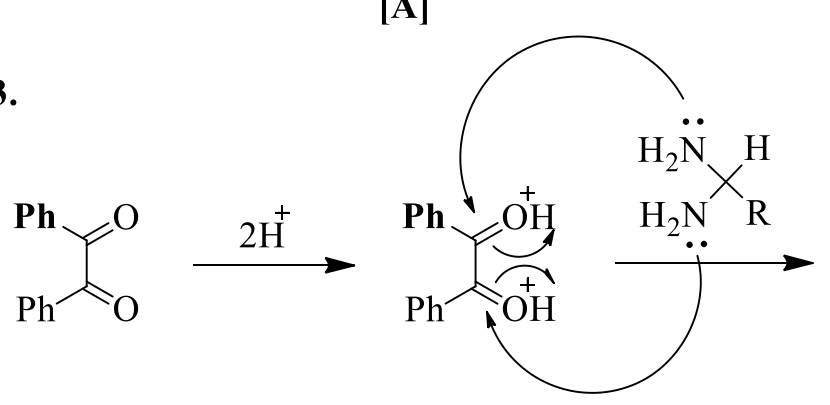<smiles>[R]C([NH3+])[N+]=[NH2+]</smiles><smiles>CCCCCCCC</smiles><smiles>[R]C1CCCCCC1</smiles><smiles>[R]c1nc(-c2ccccc2)c(-c2ccccc2)[nH]1</smiles><smiles>CC1CCC1</smiles>

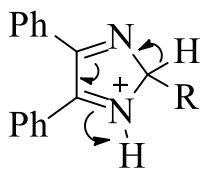<smiles></smiles><smiles>[R]C1NC2(c3ccccc3)CNC1([R])N2</smiles>

Scheme 1 Plausible mechanism for the synthesis of imidazoles catalyzed by (4-SB)T(4-SPh)PHSO

Brahmbhatt et al. [2] synthesized 3-(2,4-disubstituted phenyl)-1-(4-substituted phenyl)-4-(4,5-diphenyl-1H-imidazol-2-yl)-1H-pyrazole (Scheme 4). The antibacterial activity of these derivatives was evaluated against Staphylococcus aureus, Bacillus subtilis, Escherichia coli, and Pseudomonas aeruginosa using amikacin sulfate, ampicillin, and chloramphenicol as a reference drug. Compound $4 \mathrm{~h}$ shows the most potent activity as compared to the rest of the synthesized compounds. The conclusion of antibacterial activity was presented in (Table 4, Brahmbhatt et al. [2]).

Parab et al. [29] synthesized (Z)-4-((6-Bromo-2-chloroquinolin-3-yl) methylene)-2-phenyl-1-(2, 3, 4-trisubstituted phenyl)-1H-imidazol-5(4H)-one by using Scheme 5. The antibacterial activity of synthesized derivatives was evaluated against $E$. coli, P. aeruginosa, 


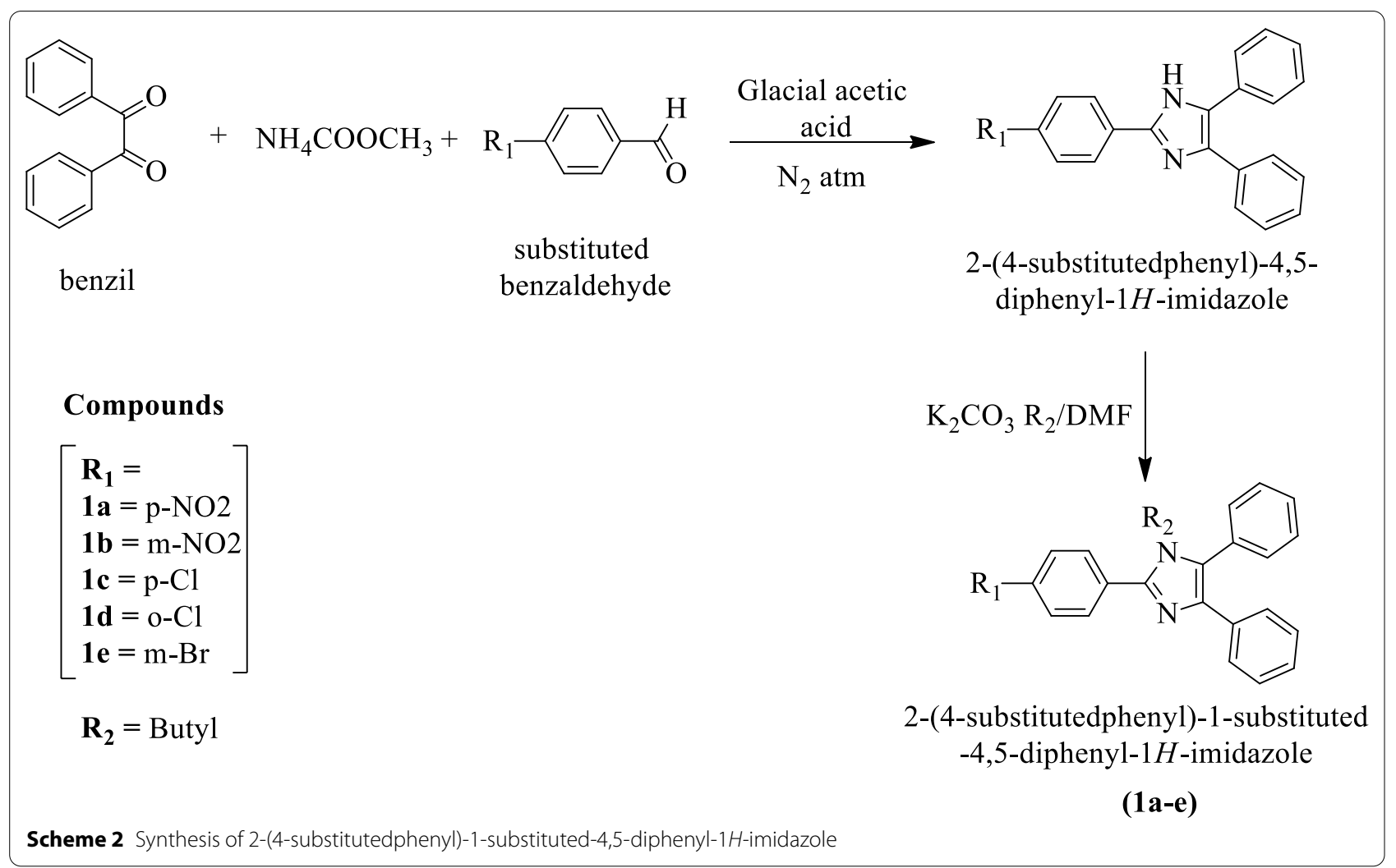

Table 2 Antibacterial activity of synthesized derivatives (1a-e)-zone of inhibition (mm,\%) Jain et al. [28]

\begin{tabular}{|c|c|c|c|c|c|c|}
\hline \multirow[t]{3}{*}{ Compounds } & \multicolumn{6}{|c|}{ Zone of inhibition } \\
\hline & \multicolumn{2}{|l|}{ S. aureus } & \multicolumn{2}{|l|}{ B. subtilis } & \multicolumn{2}{|l|}{ E. coli } \\
\hline & $50(\mu \mathrm{g} / \mathrm{mL})$ & $150(\mu \mathrm{g} / \mathrm{mL})$ & $50(\mu \mathrm{g} / \mathrm{mL})$ & $150(\mu \mathrm{g} / \mathrm{mL})$ & $50(\mu \mathrm{g} / \mathrm{mL})$ & $150(\mu \mathrm{g} / \mathrm{mL})$ \\
\hline $1 a$ & $5(23.09)$ & $9(42.85)$ & $4(19.04)$ & $8(38.09)$ & $7(33.33)$ & $9(42.85)$ \\
\hline $1 b$ & $3(14.28)$ & $7(33.33)$ & $4(19.04)$ & $7(33.33)$ & $6(28.57)$ & $9(42.85)$ \\
\hline $1 c$ & $5(23.09)$ & $6(28.57)$ & $6(28.57)$ & $7(33.33)$ & $5(23.09)$ & $8(38.09)$ \\
\hline $1 d$ & $5(23.09)$ & $6(28.57)$ & $6(28.57)$ & $6(28.57)$ & $5(23.09)$ & $8(38.09)$ \\
\hline 1e & $4(19.04)$ & $7(33.33)$ & $4(19.04)$ & $7(33.33)$ & $5(23.09)$ & $8(38.09)$ \\
\hline Norfloxacin* & 21 & - & 21 & - & 21 & - \\
\hline
\end{tabular}

Norfloxacin* Norfloxacin at concentration $50(\mu \mathrm{g} / \mathrm{mL})$

B. subtilis, and B. megaterium by agar cup borer method using streptomycin as a reference drug. The antimycotic potential was evaluated for these derivatives against Candida albicans and Aspergillus niger using imidil as a reference drug and the conclusion of activity was presented in (Table 5, Parab et al. [29]).
Sharma et al. [17] synthesized 2,3-disubstituted-3, 4-dihydroimidazo [4,5-b] indole (Scheme 6) and evaluated for antibacterial activity against Staphylococcus aureus, Bacillus subtilis, Escherichia coli, and Klebsiella pneumoniae by Kirby-Bauer disc technique using ciprofloxacin as reference drug. The conclusion 


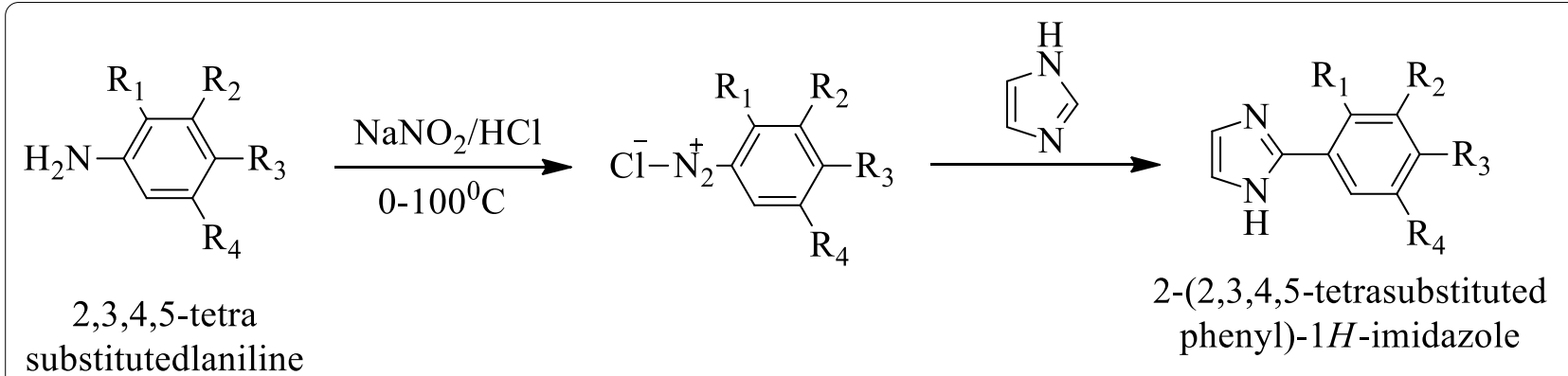
substitutedlaniline<smiles>[R4]c1cc(-c2nccn2C(=O)c2cccnc2)c([R])c([R4])c1[R]</smiles>

pyridin-3-yl(2-(2,3,4,5-tetrasubstituted phenyl)-1 $H$-imidazol-1-yl)methanone

$(2 \mathrm{a}-\mathrm{k})$

Scheme 3 Synthesis of pyridin-3-yl(2-(2,3,4,5-tetrasubstitutedphenyl)-1H-imidazol-1-yl)methanone

Table 3 Antimicrobial activity of titled compounds (2a-k) Narasimhan et al. [1]

\begin{tabular}{|c|c|c|c|c|c|}
\hline \multirow[t]{2}{*}{ Compounds } & \multicolumn{5}{|c|}{$\mathrm{MIC}(\mu \mathrm{M} / \mathrm{mL})$} \\
\hline & S. aureus & B. subtilis & E. coli & C. albicans & A. niger \\
\hline $2 a$ & 0.012 & 0.003 & 0.003 & 0.025 & 0.050 \\
\hline $2 b$ & ND & ND & ND & 0.022 & 0.005 \\
\hline $2 c$ & ND & ND & ND & 0.005 & 0.005 \\
\hline $2 d$ & 0.044 & 0.044 & 0.044 & 0.022 & 0.044 \\
\hline $2 e$ & 0.022 & 0.044 & 0.006 & 0.022 & 0.044 \\
\hline $2 f$ & 0.044 & 0.044 & 0.011 & 0.342 & 0.044 \\
\hline $2 g$ & ND & ND & ND & 0.004 & 0.019 \\
\hline $2 h$ & 0.010 & 0.010 & 0.040 & 0.020 & 0.040 \\
\hline $2 i$ & 0.040 & 0.002 & 0.040 & 0.020 & 0.040 \\
\hline $2 j$ & 0.013 & 0.005 & 0.002 & 0.025 & 0.025 \\
\hline $2 k$ & 0.002 & 0.002 & 0.002 & 0.020 & 0.040 \\
\hline Ciprofloxacin & 0.004 & 0.004 & 0.004 & - & - \\
\hline Fluconazole & - & - & - & 0.005 & 0.005 \\
\hline
\end{tabular}




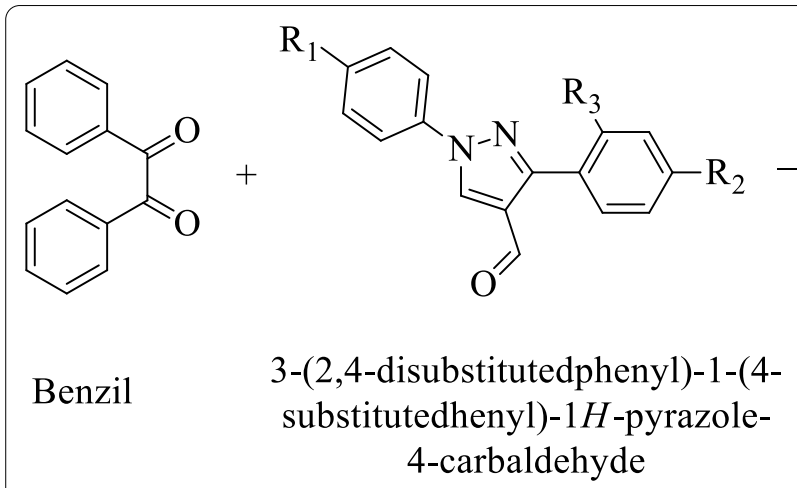

(3a-n)<smiles>Fc1cc(Br)ccc1-c1nn(-c2ccc(Cl)cc2)cc1-c1nc(-c2ccccc2)c(-c2ccccc2)[nH]1</smiles>

3-(2,4-disubstitutedphenyl)-1-(4substitutedphenyl)-4-(4,5-diphenyl- $1 H$ imidazol-2-yl)-1 $H$-pyrazole

(4a-n)

Scheme 4 Synthesis of 3-(2,4-disubstitutedphenyl)-1-(4-substitutedphenyl)-4-(4,5-diphenyl-1 H-imidazol-2-yl)-1 H-pyrazole

of antimicrobial potential was presented in (Table 6, Sharma et al. [17]).

Ahsan et al. [30] synthesized N-(4-substituted phenyl)-2(2-(2-(2-hydroxyphenyl)-4, 5-diphenyl-1H-imidazol-1-yl)

Table 4 Antibacterial activity of tri-substituted imidazole derivatives (4a-n) Brahmbhatt et al. [2]

\begin{tabular}{|c|c|c|c|c|}
\hline \multirow[t]{3}{*}{ Compounds } & \multicolumn{4}{|c|}{ Antibacterial activity (MIC in $(\mu \mathrm{g} / \mathrm{mL}$ ) } \\
\hline & \multicolumn{2}{|c|}{ Gram negative bacteria } & \multicolumn{2}{|c|}{$\begin{array}{l}\text { Gram positive } \\
\text { bacteria }\end{array}$} \\
\hline & E. coli & P. aeruginosa & B. subtilis & S. aureus \\
\hline $4 a$ & 125.0 & 125.0 & 125.0 & 125.0 \\
\hline $4 b$ & 125.0 & 125.0 & 125.0 & 125.0 \\
\hline $4 c$ & 125.0 & 125.0 & 125.0 & 125.0 \\
\hline $4 d$ & 125.0 & 125.0 & 125.0 & 125.0 \\
\hline $4 e$ & 125.0 & 125.0 & 125.0 & 125.0 \\
\hline $4 f$ & 125.0 & 125.0 & 125.0 & 125.0 \\
\hline $4 g$ & 125.0 & 125.0 & 125.0 & 125.0 \\
\hline $4 \mathrm{~h}$ & 125.0 & 125.0 & 31.0 & 63.0 \\
\hline $4 \mathrm{i}$ & 125.0 & 125.0 & 125.0 & 125.0 \\
\hline $4 \mathrm{j}$ & 125.0 & 125.0 & 125.0 & 125.0 \\
\hline $4 k$ & 125.0 & 125.0 & 125.0 & 125.0 \\
\hline 41 & 125.0 & 125.0 & 125.0 & 125.0 \\
\hline $4 m$ & 125.0 & 125.0 & 125.0 & 125.0 \\
\hline $4 n$ & 125.0 & 63.0 & 125.0 & 125.0 \\
\hline Amikacin sulphate & 2.44 & 9.77 & 9.77 & 9.77 \\
\hline Ampicillin & 100 & 100 & - & 250 \\
\hline Chloramphenicol & 50 & 50 & - & 50 \\
\hline
\end{tabular}

Values written in italic signify the best antibacterial activity acetyl) hydrazine carbothioamide (Scheme 7). The antibacterial activity of synthesized derivatives was evaluated against Escherichia coli, Bacillus subtilis, and Staphylococcus aureus using Ofloxacin as a reference drug. The antimycotic potential was evaluated for these derivatives against $C$. albicans using Voriconazole as a positive control. Compounds $8 \mathrm{a}, 8 \mathrm{~b}$, and $8 \mathrm{~d}$ showed good antifungal activity against $C$. albicans. The conclusion of antimicrobial activity was presented in (Table 7, Ahsan et al. [30]).

Bhade et al. [18] synthesized 2,4-dichloro-6-(2substituted-2,5-dihydro-1H-imidazol-4-yl)phenol, 6-(3, 5dichloro-2-hydroxyphenyl)-2-substituted-2H-imidazo [1,2-a]imidazol-3(5H)-one, 1-acetyl-4-(3, 5-dichloro-2hydroxyphenyl)-1H-imidazol-2(5H)-one, (Z)-4-(3,5-dichloro2-hydroxyphenyl)-1-(3-(2, 3-dichlorophenyl) acryloyl)$1 \mathrm{H}$-imidazol-2 $(5 \mathrm{H})$-one and 4-(3,5-dichloro-2-hydroxy phenyl)-1-(5-(2,3-dichlorophenyl)-4,5-dihydro-1Hpyrazol-3-yl)-1H-imidazol-2(5H)-one by using (Scheme 8 ). The antibacterial activity of these derivatives was evaluated against Staphylococcus aureus, Staphylococcus epidermidis, Salmonella typhi and Pseudomonas aeruginosa using chloramphenicol as reference control. The conclusion of activity was presented in (Table 8, Bhade et al. [18]).

Desai et al. [31] synthesized (Z)-(4-((2-chloroquinolin-3-yl)methylene)-5-oxo-2-phenyl-4,5-dihydro1H-imidazol-1-yl)substituted carbamic (Scheme 9) and evaluated for antimicrobial potential against Staphylococcus aureus, Escherichia coli, Pseudomonas aeruginosa, and Streptococcus pyogenes by serial broth dilution method using ampicillin as a reference standard and the results were summarized in (Table 9a, Desai et al. [30]). 


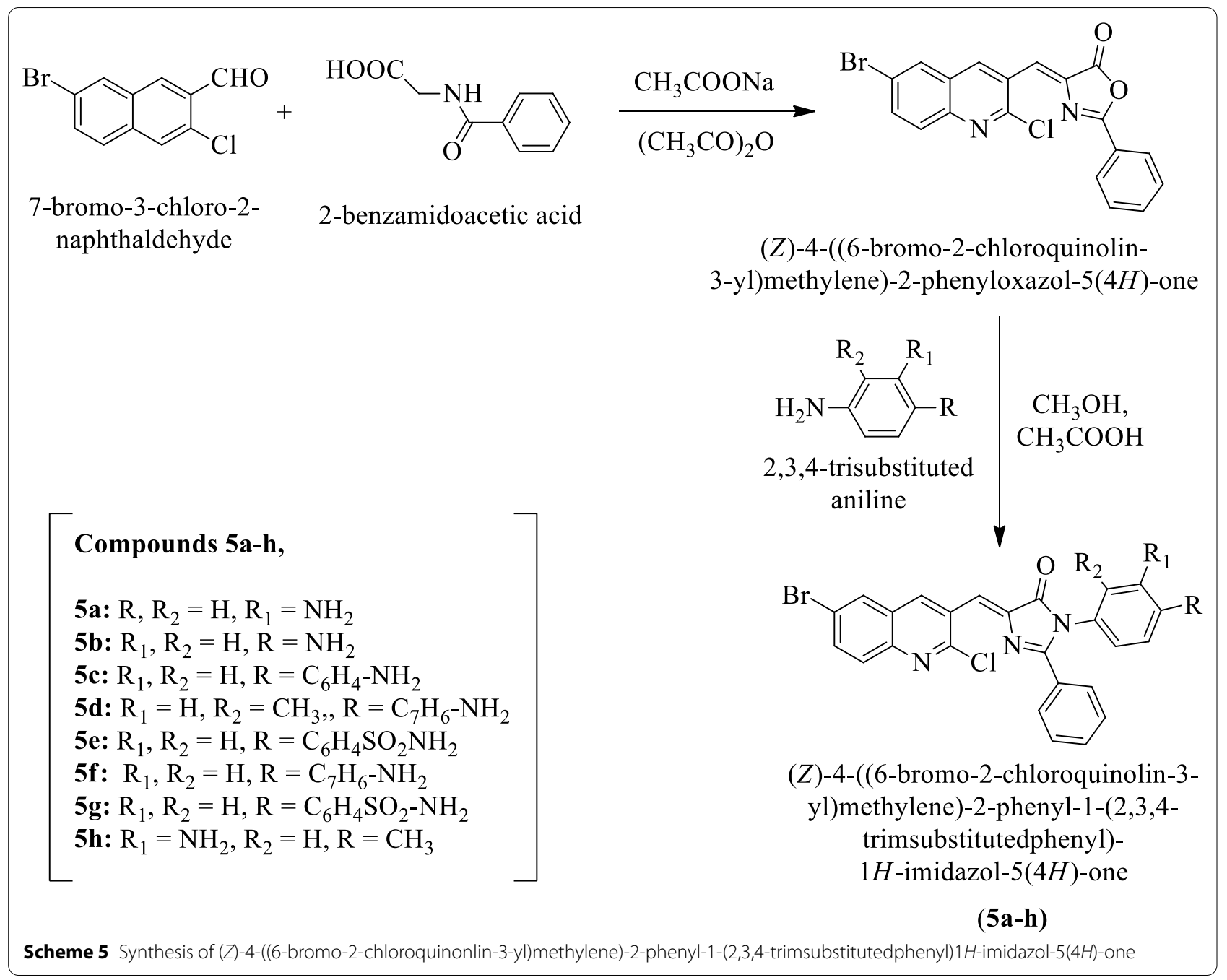

Table 5 Antimicrobial activity of synthesized compounds (5a-h) Parab et al. [29]

\begin{tabular}{|c|c|c|c|c|c|c|}
\hline \multirow[t]{2}{*}{ Compounds } & \multicolumn{6}{|c|}{ Zone of inhibition ( $\mathrm{mm}$ ) } \\
\hline & E. coli & P. aeruginosa & B. subtilis & B. megaterium & A. niger & C. albicans \\
\hline $5 a$ & 15 & 19 & 21 & 19 & 20 & 19 \\
\hline $5 b$ & 11 & 9 & 19 & 20 & 10 & 11 \\
\hline $5 c$ & 20 & 22 & 22 & 22 & 13 & 13 \\
\hline $5 d$ & 11 & 15 & 19 & 13 & 15 & 12 \\
\hline $5 e$ & 10 & 8 & 15 & 19 & 12 & 9 \\
\hline $5 f$ & 6 & 18 & 25 & 20 & 14 & 16 \\
\hline $5 g$ & 14 & 9 & 24 & 15 & 10 & 11 \\
\hline $5 h$ & 8 & 13 & 21 & 13 & 16 & 17 \\
\hline Streptomycin & 28 & 32 & 31 & 29 & 33 & 33 \\
\hline Imidil & - & - & - & - & 34 & 34 \\
\hline
\end{tabular}




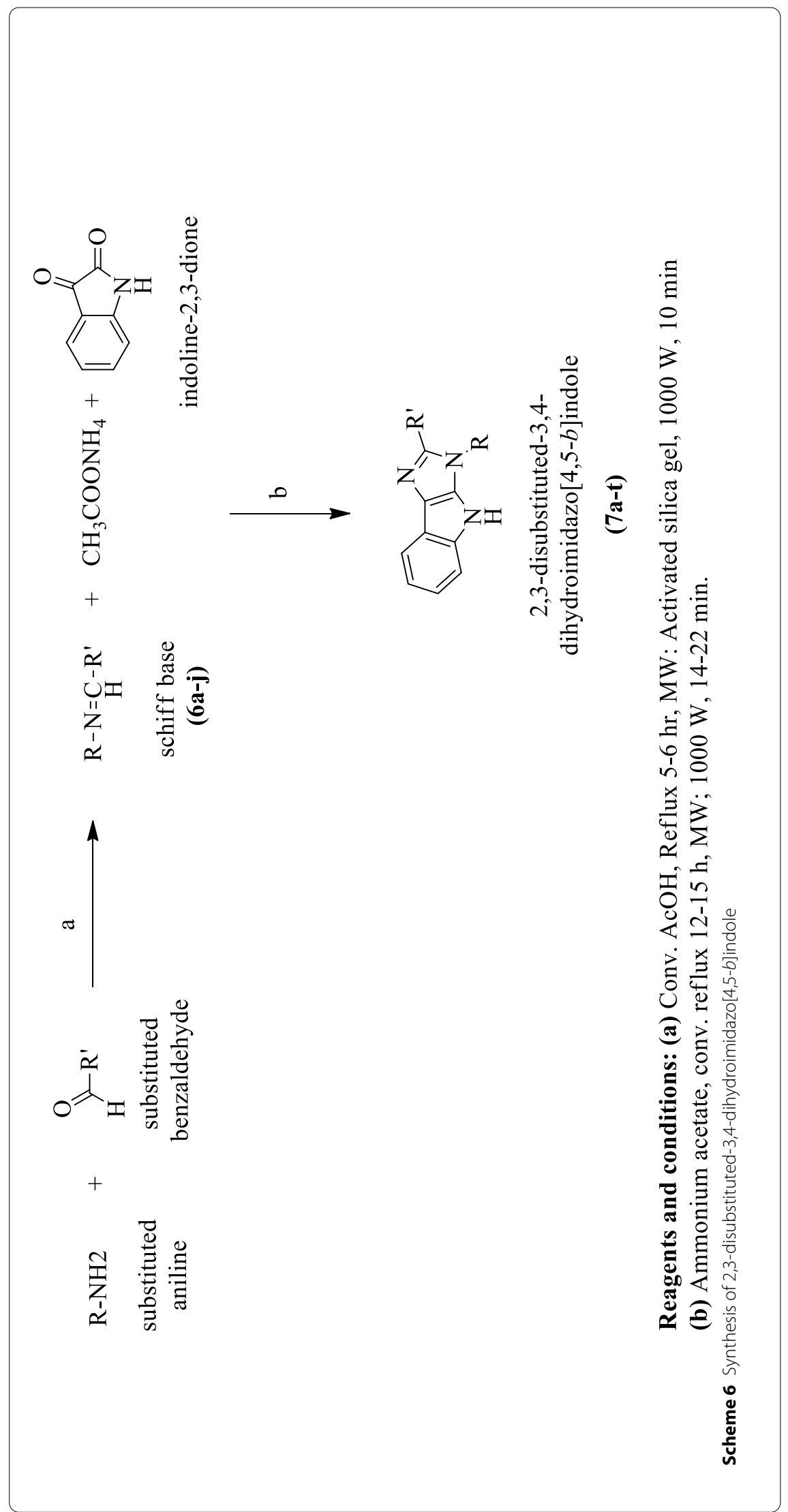


Table 6 Antimicrobial activity of the synthesized aryl imidazole compounds (7a-t) Sharma et al. [17]

\begin{tabular}{|c|c|c|c|c|}
\hline \multirow[t]{3}{*}{ Compounds } & \multicolumn{4}{|c|}{ Diameter of zone of inhibition $(\mathrm{mm})$ Bacterial strains } \\
\hline & \multicolumn{2}{|c|}{$\begin{array}{l}\text { Gram positive } \\
\text { bacteria }\end{array}$} & \multicolumn{2}{|c|}{ Gram negative bacteria } \\
\hline & S. aureus & B. subtilis & E. coli & K. pneumoniae \\
\hline $7 a$ & $5.9(50)$ & $6.9(50)$ & $7.2(50)$ & $8.1(50)$ \\
\hline $7 b$ & $5.1(25)$ & $5.5(25)$ & $8.1(50)$ & $8.9(50)$ \\
\hline $7 c$ & $8.6(25)$ & $8.4(25)$ & $9.2(12.5)$ & $9.5(12.5)$ \\
\hline $7 d$ & $13.1(50)$ & $12.5(25)$ & $11.9(25)$ & $12.5(6.2)$ \\
\hline $7 e$ & $9.1(25)$ & $8.8(50)$ & $7.6(100)$ & $7.8(100)$ \\
\hline $7 f$ & $5.7(100)$ & $5.9(100)$ & $6.6(50)$ & $6.9(50)$ \\
\hline $7 g$ & $12.5(50)$ & $12.1(25)$ & $11.9(25)$ & $11.6(25)$ \\
\hline $7 \mathrm{~h}$ & $11.9(50)$ & $11.3(25)$ & $10.9(100)$ & $10.7(50)$ \\
\hline $7 i$ & $12.1(25)$ & $13.8(50)$ & $14.3(25)$ & $12.5(50)$ \\
\hline $7 j$ & $13.1(25)$ & $12.3(25)$ & $15.4(12.5)$ & $11.8(25)$ \\
\hline $7 \mathrm{k}$ & $11.2(50)$ & $12.4(25)$ & $13.5(12.5)$ & $9.1(50)$ \\
\hline 71 & $6.2(100)$ & $7.2(100)$ & $9.2(50)$ & $7.5(50)$ \\
\hline $7 m$ & $7.2(100)$ & $8.7(50)$ & $10.2(50)$ & $10.3(25)$ \\
\hline $7 n$ & $10.3(25)$ & $12.4(12.5)$ & $14.5(6.2)$ & $13.3(12.5)$ \\
\hline 70 & $12.3(50)$ & $13.6(25)$ & $14.6(25)$ & $14.6(25)$ \\
\hline $7 p$ & $9.1(100)$ & $8.3(100)$ & $9.1(50)$ & $10.2(25)$ \\
\hline $7 q$ & $6.1(100)$ & $7.4(100)$ & $8.3(50)$ & $6.9(50)$ \\
\hline $7 r$ & $7.3(100)$ & $7.4(100)$ & $9.5(50)$ & $9.7(50)$ \\
\hline $7 \mathrm{~s}$ & $13.2(25)$ & 14.5 (12.5) & $14.6(12.5)$ & $11.5(25)$ \\
\hline $7 t$ & $12.4(25)$ & $12.7(25)$ & $13.1(50)$ & $11.1(50)$ \\
\hline Ciprofloxacin & $18(12.5)$ & $19(6)$ & $19(12.5)$ & $17(6)$ \\
\hline
\end{tabular}

Values in brackets are MIC values $(\mu \mathrm{g} / \mathrm{mL})$

The antimycotic potential of these derivatives was evaluated against $A$. niger, $C$. albicans, and $A$. clavatus using griseofulvin as a reference standard. The results of the activity were summarized in (Table 9b, Desai et al. [31]).

Shobhashana et al. [32] synthesized 6-substituted3-(4,5-diphenyl-1H-imidazol-2-yl)-2-(4-substituted phenoxy) quinoline by using (Scheme 10) and evaluated for antimicrobial activity against Bacillus subtilis, Escherichia coli, Clostridium tetani, Streptococcus pneumoniae, and Salmonella typhi by using the broth dilution method.
Ampicillin, chloramphenicol, and ciprofloxacin were used as a positive control. The antimycotic activity of these derivatives was evaluated against Candida albicans and Trichophyton rubrum using Nystatin and Griseofulvin as reference drugs. The conclusion of antimicrobial activity was presented in (Table 10a, b, Shobhashana et al. [32]).

Selvan et al. [33] developed N-(2-(1H-benzo[d]imidazol-2-yl)phenyl)substituted formimidoyl by using (Scheme 11). The disc diffusion technique was used for the determination of antimicrobial activity against $S$. aureus using ciprofloxacin as a positive control. The antimycotic activity of these derivatives was evaluated against $A$. niger using Nystatin as a reference drug and the conclusion of antimicrobial potential was presented in (Table 11, Selvan et al. [33]).

Zala et al. [8] synthesized 2-(substituted amino)-1-(2,4,5triphenyl-1H-imidazol-1-yl) ethanone (Scheme 12) and evaluated for antimicrobial potential against Staphylococcus aureus and Escherichia coli using ciprofloxacin as a reference drug. The antimycotic potential of these derivatives was evaluated against $C$. albicans using Clotrimazole as a reference drug. The conclusion of antibacterial activity was presented in (Table 12, Zala et al. [8]).

Yadav et al. [34] synthesized 2-((1H-benzo[d]imidazol-2-yl)thio)-N-(4-oxo-2-(2,3,4,5,6-Penta substituted phenyl)thiazolidin-3-yl)acetamide and 2-((1H-benzo[d] imidazol-2-yl)thio)-N-(2-substituted-4-oxothiazolidin3 -yl) acetamide by using (Scheme 13). The antibacterial activity of these derivatives was evaluated against different bacterial strains (Staphylococcus aureus, Escherichia coli, and Bacillus subtilis) using Norfloxacin as a reference drug. The antimycotic activity of these derivatives was evaluated against different fungal (Candida albicans and Aspergillus niger) strains using Fluconazole as a reference drug. The conclusion of the activity was presented in (Table 13, Yadav et al. [34]).

\section{Anticancer activity}

Yurttas et al. [35] developed 2-((1-((4-substituted phenyl) amino)-4,5-dimethyl-1H-imidazol-2-yl)thio)-N-(6-substi 


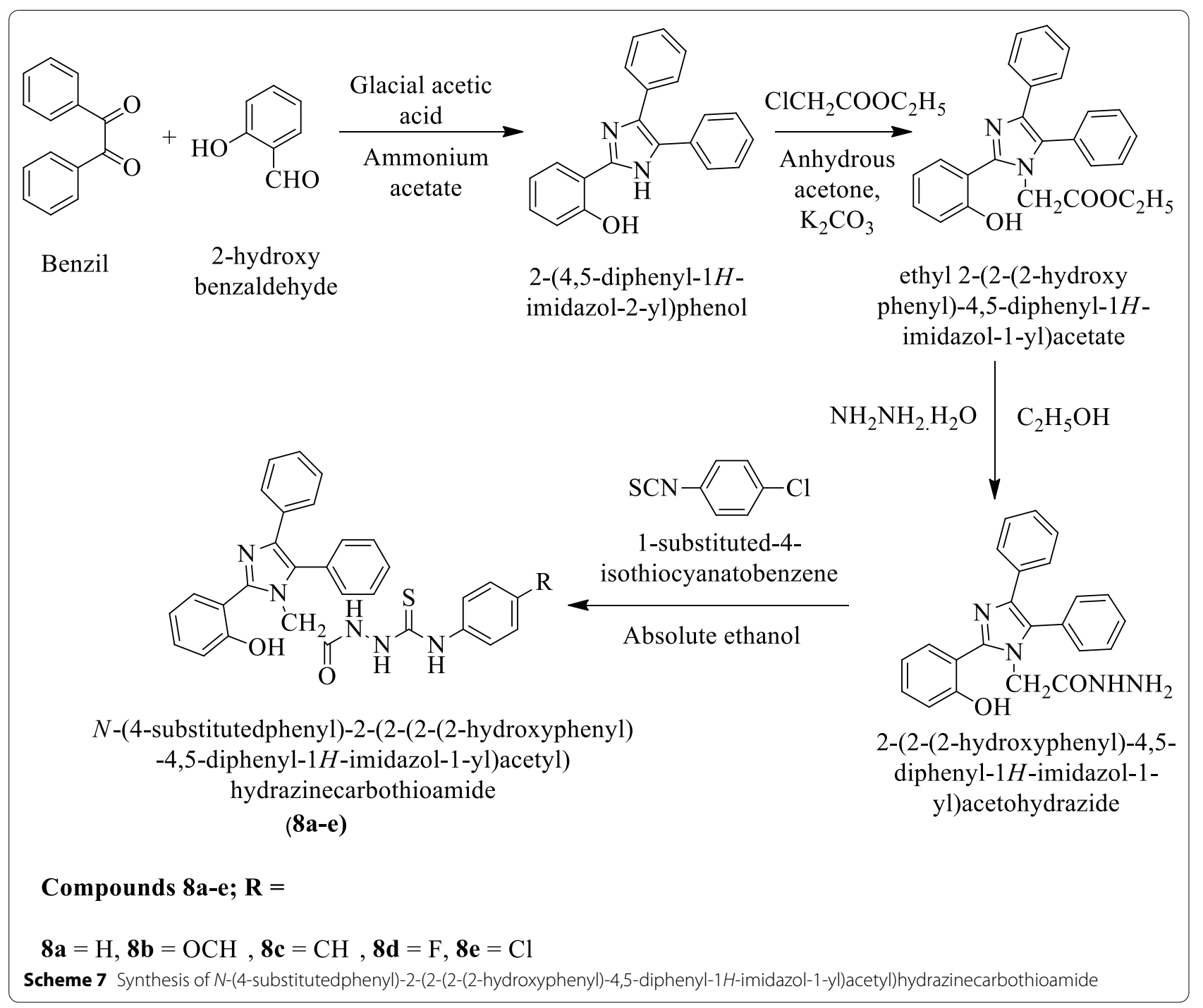

Table 7 Antibacterial and antifungal activity of titled compounds (8a-8e) Ahsan et al. [30]

\begin{tabular}{|c|c|c|c|c|c|c|c|c|}
\hline \multirow[t]{3}{*}{ Compounds } & \multicolumn{6}{|c|}{ Antibacterial activity } & \multirow{2}{*}{\multicolumn{2}{|c|}{$\begin{array}{l}\text { Antifungal activity } \\
\text { C. albicans }\end{array}$}} \\
\hline & \multicolumn{2}{|l|}{ E. coli } & \multicolumn{2}{|l|}{ B. subtilis } & \multicolumn{2}{|l|}{ S. aureus } & & \\
\hline & $\begin{array}{l}\text { Zone } \\
\text { of inhibition } \\
(\mathrm{mm})\end{array}$ & $\%$ inhibition & $\begin{array}{l}\text { Zone } \\
\text { of inhibition } \\
(\mathrm{mm})\end{array}$ & $\%$ inhibition & $\begin{array}{l}\text { Zone } \\
\text { of inhibition } \\
(\mathrm{mm})\end{array}$ & \% inhibition & $\begin{array}{l}\text { Zone } \\
\text { of inhibition } \\
(\mathrm{mm})\end{array}$ & $\%$ inhibition \\
\hline $8 a$ & 22 & 61.11 & 14 & 43 & 14 & 48 & 20 & 75 \\
\hline $8 b$ & 22 & 61.11 & 15 & 47 & 21 & 72 & 20 & 69 \\
\hline $8 c$ & 23 & 67 & 15 & 47 & 20 & 69 & 17 & 58.6 \\
\hline $8 d$ & 22.5 & 62.5 & 20 & 62 & 19 & 65.5 & 20 & 69 \\
\hline $8 e$ & 19 & 52 & 12 & 50 & 22 & 75 & 20 & 68.5 \\
\hline Ofloxacin & 36 & 100 & 32 & 100 & 29 & 100 & - & - \\
\hline Voriconazole & - & - & - & - & - & - & 29 & 100 \\
\hline
\end{tabular}




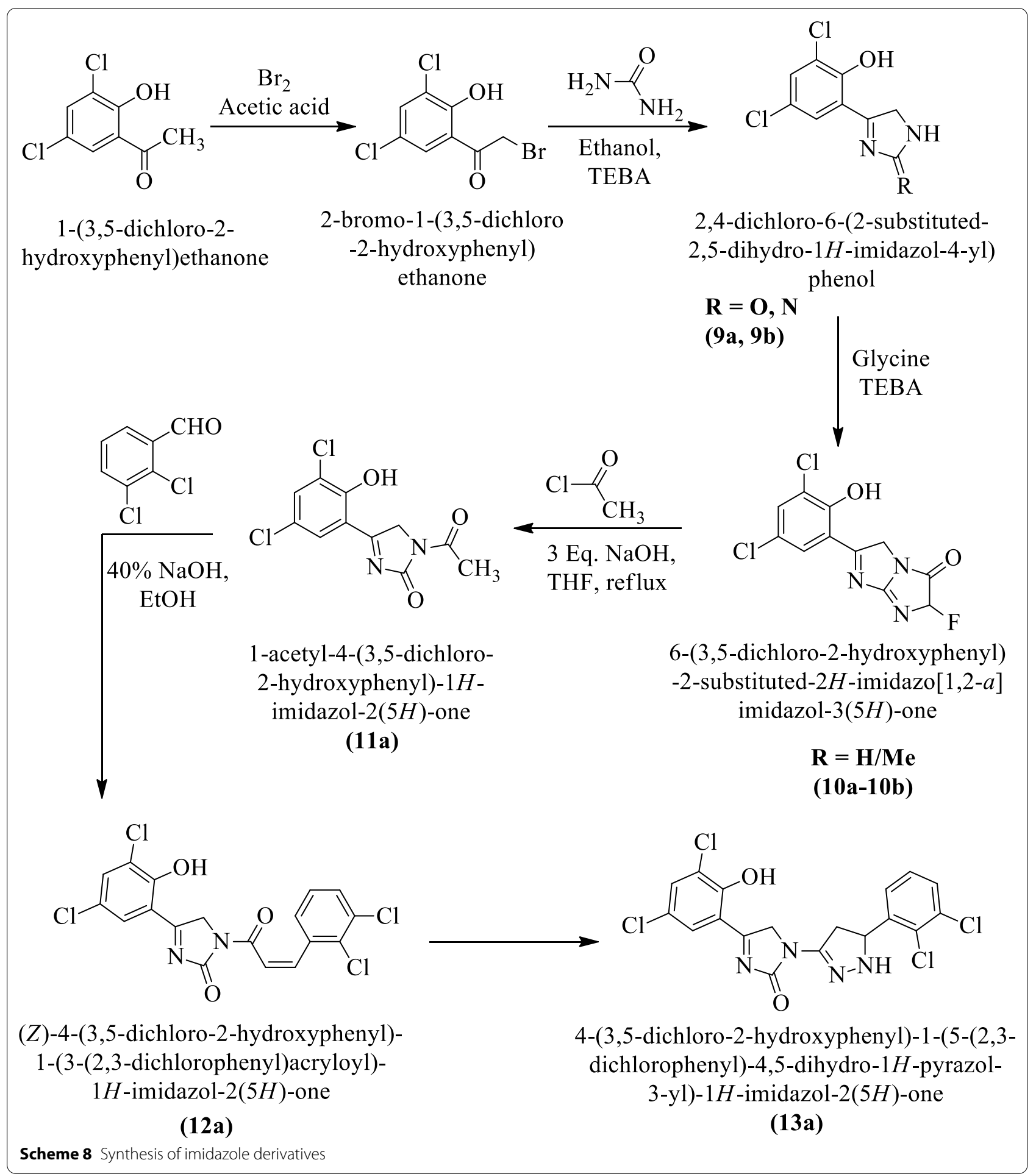




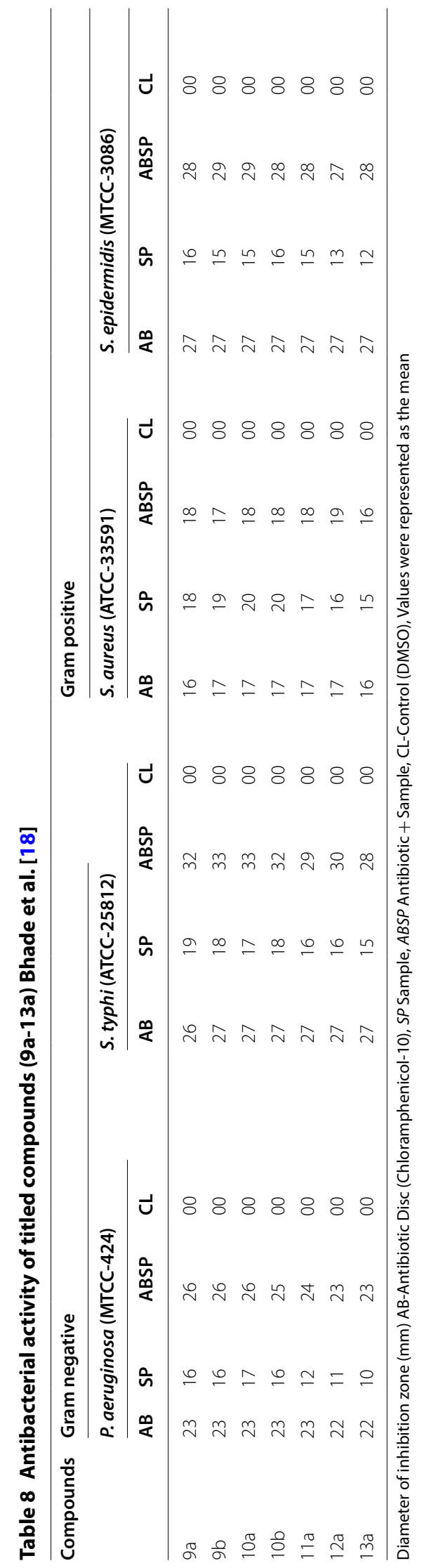




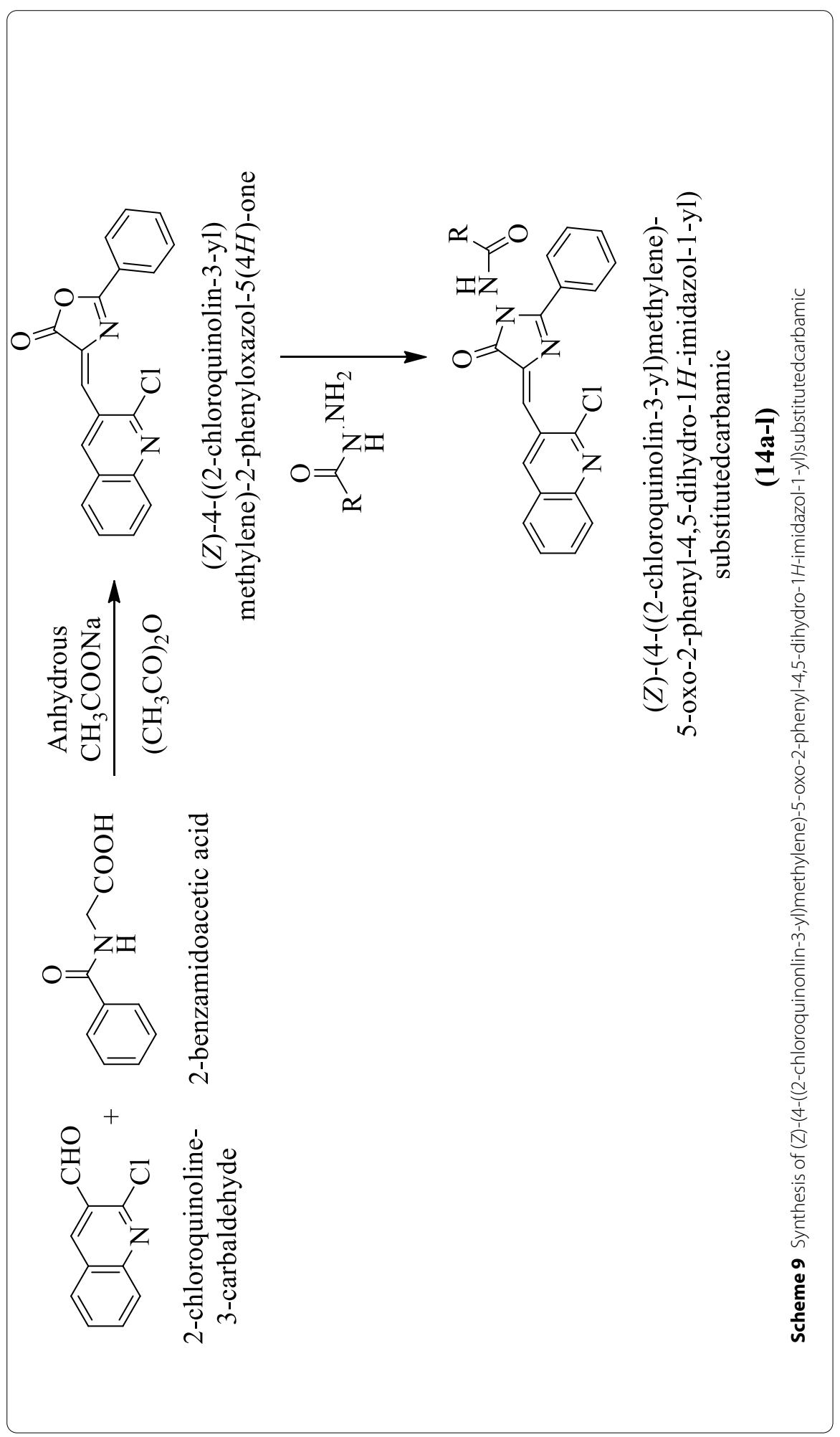


Table 9 (a) Antibacterial activity of the synthesized derivatives (14a-I); (b) Antifungal activity of titled compounds (14a-I) Desai et al. [31]

\begin{tabular}{|c|c|c|c|c|c|}
\hline \multirow[t]{2}{*}{ Compounds } & \multirow[t]{2}{*}{$\mathbf{R}$} & \multicolumn{4}{|l|}{$\mathrm{MIC}(\mu \mathrm{g} / \mathrm{mL}) \pm \mathrm{SD}$} \\
\hline & & E. coli MTCC-443 & $\begin{array}{l}\text { P. aeruginosa } \\
\text { MTCC-1688 }\end{array}$ & S. aureus MTCC-96 & S. pyogenesMTCC-442 \\
\hline $14 a$ & $-\mathrm{C}_{6} \mathrm{H}_{5}$ & $100 \pm 2.03^{* *}$ & $500 \pm 2.64^{*}$ & $1000 \pm 3.78$ & $500 \pm 2.64$ \\
\hline $14 b$ & $\mathrm{C}_{6} \mathrm{H}_{5}-\mathrm{CH}_{2^{-}}$ & $500 \pm 3.46^{*}$ & $500 \pm 3.46$ & $250 \pm 3.21^{* *}$ & $250 \pm 3.04^{* * *}$ \\
\hline $14 c$ & $-3-\mathrm{Cl}-\mathrm{C}_{6} \mathrm{H}_{4}$ & $50 \pm 2.64^{* * *}$ & $100 \pm 1.21^{* *}$ & $200 \pm 2.08^{*}$ & $1000 \pm 4.51$ \\
\hline $14 d$ & $-4-\mathrm{Cl}-\mathrm{C}_{6} \mathrm{H}_{4}$ & $25 \pm 1^{*}$ & $100 \pm 1.51^{*}$ & $200 \pm 2.08^{* *}$ & $50 \pm 2.64^{* *}$ \\
\hline $14 \mathrm{e}$ & $-2,5-(\mathrm{Cl})_{2}-\mathrm{C}_{6} \mathrm{H}_{3}$ & $100 \pm 1$ & $250 \pm 2.51^{* *}$ & $1000 \pm 4.04$ & $1000 \pm 2.51^{*}$ \\
\hline $14 f$ & $-4-\mathrm{F}-\mathrm{C}_{6} \mathrm{H}_{4}$ & $200 \pm 1.62^{*}$ & $100 \pm 1.60$ & $100 \pm 2.78^{* *}$ & $1000 \pm 3.78^{* *}$ \\
\hline $14 \mathrm{~g}$ & $-3-\mathrm{NO}_{2}-\mathrm{C}_{6} \mathrm{H}_{4}$ & $100 \pm 1^{* *}$ & $100 \pm 1.72$ & $500 \pm 3.05$ & $250 \pm 2.51^{* * *}$ \\
\hline $14 \mathrm{~h}$ & $-4-\mathrm{NO}_{2}-\mathrm{C}_{6} \mathrm{H}_{4}$ & $25 \pm 1.62^{* * *}$ & $50 \pm 1.05^{*}$ & $250 \pm 2.16^{*}$ & $100 \pm 1.78^{* *}$ \\
\hline $14 i$ & $-2-\mathrm{OH}-\mathrm{C}_{6} \mathrm{H}_{4}$ & $100 \pm 2.15^{*}$ & $100 \pm 1^{* * *}$ & $100 \pm 2.04^{*}$ & $500 \pm 4.50$ \\
\hline $14 \mathrm{j}$ & $-3-\mathrm{OH}-\mathrm{C}_{6} \mathrm{H}_{4}$ & $100 \pm 2.05^{*}$ & $50 \pm 1.16^{* *}$ & $500 \pm 4.50$ & $200 \pm 2.05^{*}$ \\
\hline $14 k$ & $-2-\mathrm{OH}, 4-\mathrm{Cl}-\mathrm{C}_{6} \mathrm{H}_{3}$ & $200 \pm 2.21^{*}$ & $100 \pm 2.15^{* *}$ & $250 \pm 2.64^{* *}$ & $500 \pm 3.08$ \\
\hline 141 & $\mathrm{C}_{5} \mathrm{H}_{4} \mathrm{~N}$ & $500 \pm 3.05^{* *}$ & $500 \pm 3.78$ & $250 \pm 3.21^{*}$ & $100 \pm 1.51^{*}$ \\
\hline Ampicillin & & $100 \pm 2.05$ & $100 \pm 1.0$ & $250 \pm 1.52$ & $100 \pm 2.06$ \\
\hline
\end{tabular}

(b)

\begin{tabular}{|c|c|c|c|c|}
\hline \multirow[t]{2}{*}{ Compounds } & \multirow[t]{2}{*}{$\mathbf{R}$} & \multicolumn{3}{|l|}{$\operatorname{MIC}(\mu \mathrm{g} / \mathrm{mL}) \pm S D$} \\
\hline & & C. albicans MTCC-227 & A. niger MTCC-282 & A. clavatus MTCC-1323 \\
\hline $14 a$ & $-\mathrm{C}_{6} \mathrm{H}_{5}$ & $500 \pm 2.64^{*}$ & $500 \pm 3.05^{*}$ & $1000 \pm 3.21$ \\
\hline $14 b$ & $\mathrm{C}_{6} \mathrm{H}_{5}-\mathrm{CH}_{2}^{-}$ & $1000 \pm 1.04^{* *}$ & $1000 \pm 2.51^{* *}$ & $500 \pm 4.05^{*}$ \\
\hline $14 c$ & $-3-\mathrm{Cl}-\mathrm{C}_{6} \mathrm{H}_{4}$ & $100 \pm 1.51^{*}$ & $1000 \pm 4.50$ & $100 \pm 1.64^{*}$ \\
\hline $14 d$ & $-4-\mathrm{Cl}-\mathrm{C}_{6} \mathrm{H}_{4}$ & $200 \pm 2.64^{*}$ & $100 \pm 1.21^{* *}$ & $500 \pm 4.16$ \\
\hline $14 \mathrm{e}$ & $-2,5-(\mathrm{Cl})_{2}-\mathrm{C}_{6} \mathrm{H}_{3}$ & $100 \pm 2.51^{* *}$ & $500 \pm 2.08^{* * *}$ & $500 \pm 3.78^{* *}$ \\
\hline $14 f$ & $-4-\mathrm{F}-\mathrm{C}_{6} \mathrm{H}_{4}$ & $100 \pm 1.78^{*}$ & $1000 \pm 3.05$ & $100 \pm 2.78^{* * *}$ \\
\hline $14 \mathrm{~g}$ & $-3-\mathrm{NO}_{2}-\mathrm{C}_{6} \mathrm{H}_{4}$ & $200 \pm 3.51$ & $500 \pm 4.05^{*}$ & $100 \pm 1.51^{*}$ \\
\hline $14 \mathrm{~h}$ & $-4-\mathrm{NO}_{2}-\mathrm{C}_{6} \mathrm{H}_{4}$ & $100 \pm 3.78^{* *}$ & $100 \pm 1^{* * * *}$ & $200 \pm 3.05^{* *}$ \\
\hline $14 \mathrm{i}$ & $-2-\mathrm{OH}-\mathrm{C}_{6} \mathrm{H}_{4}$ & $500 \pm 4.50^{*}$ & $250 \pm 3.78^{* *}$ & $500 \pm 4.58$ \\
\hline $14 j$ & $-3-\mathrm{OH}-\mathrm{C}_{6} \mathrm{H}_{4}$ & $1000 \pm 2.05^{* * *}$ & $100 \pm 2.05^{* * *}$ & $500 \pm 3.21^{* *}$ \\
\hline $14 k$ & $-2-\mathrm{OH}, 4-\mathrm{Cl}-\mathrm{C}_{6} \mathrm{H}_{3}$ & $500 \pm 2.08$ & $250 \pm 2.05$ & $500 \pm 3.46$ \\
\hline 141 & $\mathrm{C}_{5} \mathrm{H}_{4} \mathrm{~N}$ & $200 \pm 3.51^{* *}$ & $500 \pm 2.64^{*}$ & $100 \pm 1.12^{*}$ \\
\hline Griseofulvin & & $500 \pm 2.58$ & $100 \pm 1$ & $100 \pm 1.15$ \\
\hline
\end{tabular}

$\pm \mathrm{SD}=$ Standard deviation

* Significant $P<0.05$

** Moderately significant $P<0.01$

*** Extremely significant $P<0.001$ 


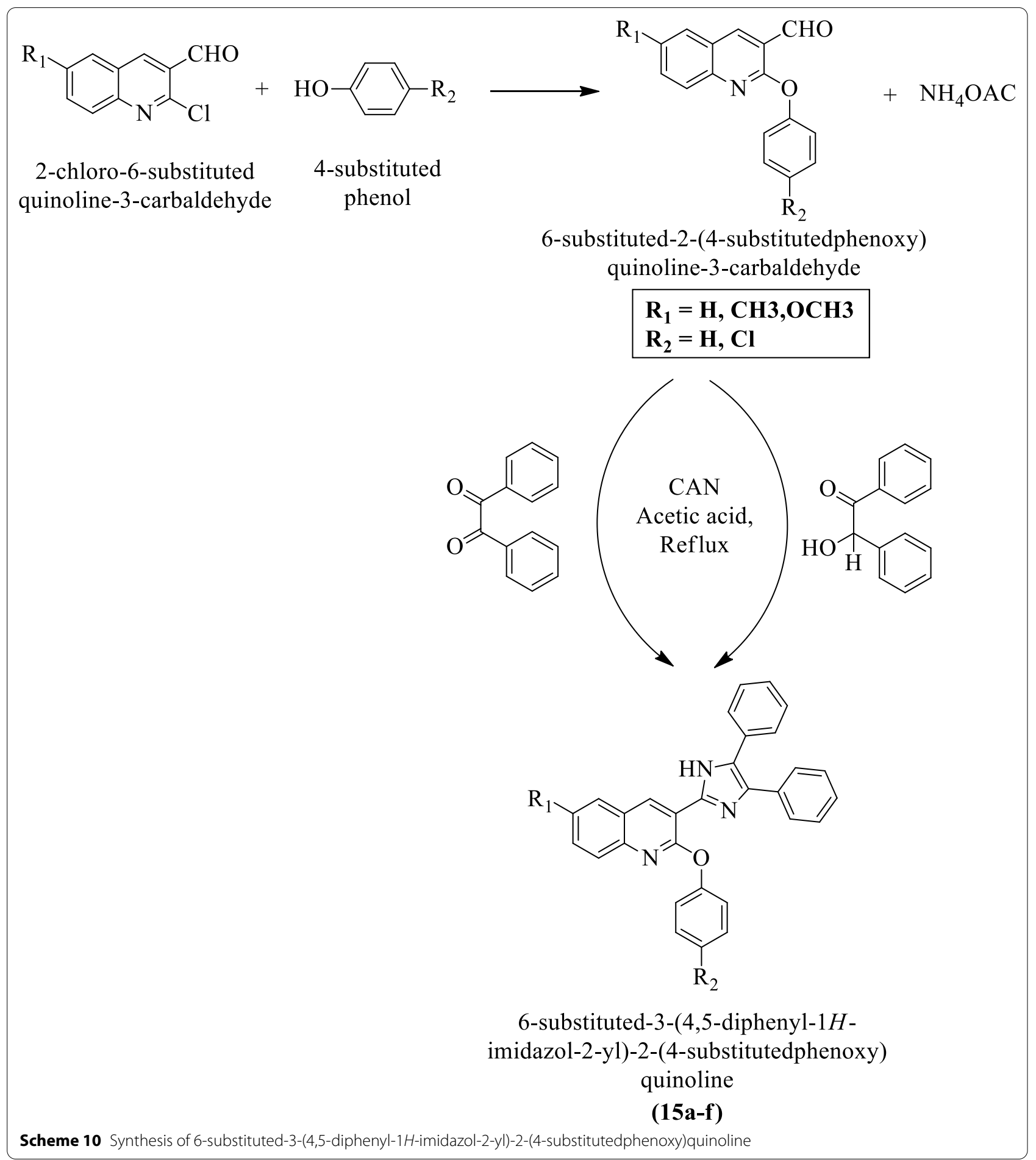


Table 10 (a) Antibacterial activity of the synthesized compounds (15a-f); (b) Antifungal activity of the synthesized compounds (15a-f) Shobhashana et al. [32]

\begin{tabular}{|c|c|c|c|c|c|c|}
\hline \multirow[t]{3}{*}{ Compounds } & \multicolumn{6}{|c|}{ Minimum inhibitory concentration in $\mu \mathrm{g} / \mathrm{mL}$} \\
\hline & \multicolumn{6}{|c|}{ Antibacterial activity } \\
\hline & \multicolumn{3}{|c|}{ Gram positive bacteria } & \multicolumn{3}{|c|}{ Gram negative bacteria } \\
\hline $15 a$ & 100 & 250 & 500 & 100 & 250 & 250 \\
\hline $15 b$ & 250 & 500 & 250 & 250 & 200 & 500 \\
\hline $15 c$ & 62.5 & 100 & 500 & 62.5 & 200 & 250 \\
\hline $15 d$ & 250 & 100 & 125 & 100 & 125 & 100 \\
\hline $15 e$ & 500 & 500 & 500 & 250 & 100 & 500 \\
\hline $15 f$ & 100 & 250 & 100 & 100 & 100 & 250 \\
\hline Ampicillin & 250 & 250 & 100 & 100 & 100 & 100 \\
\hline Chloramphenicol & 50 & 50 & 50 & 50 & 50 & 50 \\
\hline Ciprofloxacin & 50 & 100 & 50 & 25 & 25 & 25 \\
\hline
\end{tabular}

(b)

\begin{tabular}{lcr}
\hline Compounds & \multicolumn{1}{l}{ MIC } & \multicolumn{1}{c}{ T.rubrum } \\
\cline { 2 - 3 } & Antifungal activity & MTC296 \\
\cline { 2 - 3 } & C.albicans MTCC227 & 1000 \\
\hline $15 a$ & $>1000$ & $>1000$ \\
$15 b$ & 500 & 1000 \\
$15 c$ & 1000 & $>000$ \\
$15 d$ & 1000 & $>1000$ \\
$15 e$ & 1000 & 1000 \\
$15 f$ & 500 & 500 \\
Nystatin & 100 & 500 \\
Griseofulvin & 500 & \\
\hline
\end{tabular}

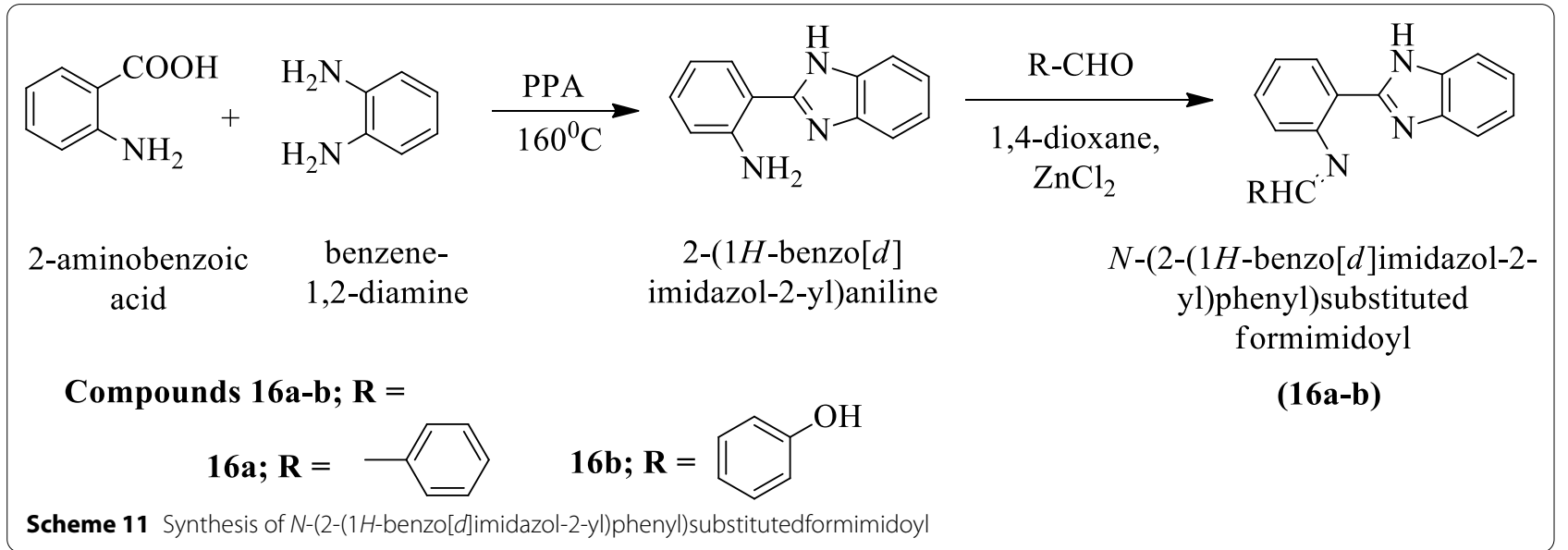


Table 11 Antimicrobial activity of titled compounds (16ab) Selvan et al. [33]

\begin{tabular}{lll}
\hline Compounds & \multicolumn{2}{l}{ Zone of inhibition in $\mathbf{m m}$} \\
\cline { 2 - 3 } & Antibacterial activity & Antifungal activity \\
& S. aureus (NCIM-2079) & A. niger (NCIM-105) \\
\hline $16 \mathrm{a}$ & 22 & 18 \\
$16 \mathrm{~b}$ & 16 & 20 \\
Solvent & - & - \\
Ciprofloxacin & 35 & - \\
Nystatin & - & 35 \\
\hline
\end{tabular}

Standard-Ciprofloxacin $5 \mathrm{mg} /$ disc for bacteria. Nystatin 100 units/disc for fungi; Solvent-DMSO

tutedbenzo[d]thiazol-2-yl)acetamide by using (Scheme 14) and evaluated for antitumor potential by MTT assay against two different cancer cell lines such as C6 (rat glioma) and HepG2 (human liver) using cisplatin as a reference drug. Among the synthesized derivatives compound $20 \mathrm{~g}$ shows good cytotoxic potential. The conclusion of antitumor potential was presented in (Table 14, Yurttas et al. [35]).

Hsieh et al. [25] synthesized (E)-1-(1-allyl-1H-benzo[d] imidazol-2-yl)-3-(4-substituted phenyl) prop-2-en-1one by using (Scheme 15) and evaluated for anticancer activity against different cell lines such as A549, MCF7, HepG2, and OVCAR-3 by MTT assay using cisplatin as a reference drug. The conclusion of anticancer activity was presented in (Table 15, Hsieh et al. [25]).

Roopashree et al. [36] synthesized 2-(5-butyl-3-chloro1-substituted-1H-pyrrol-2-yl)-1H-benzo[d]imidazole (Scheme 16) and evaluated for antitumor activity against HeLa cancer cell line by using MTT assay. Each compound was tested to calculate the inhibitory concentration and the results of the activity were presented in (Table 16, Roopashree et al. [36]).

Romagnoli et al. [37] developed 2-substituted-1-(3,4,5trimethoxyphenyl)-1H-imidazole (Scheme 17) and evaluated for anticancer activity against different cancer cell lines such as HeLa, HT-29, A549, MCF-7, Jurkat, and HL-60 using C-A4 as a reference standard. Compounds 28k, 28n, and 28o showed maximum cytotoxicity as compared to others. The conclusion of antitumor potential was presented in (Table 17, Romagnoli et al. [37]).

Rajendran et al. [38] synthesized 1-substituted-2-(5substituted-1-phenyl-1-H-pyrazol-3-yl)-1H-benzo[d] imidazole and 4-(1-chloro-1H-benzo[d]imidazol-2-yl)6-fluoropyrimidin-2-amine by using (Scheme 18) and evaluated for antitumor potential against different cell lines such as MCF-7 and CaCo-2 using Fluorouracil as reference drug. Each compound was tested to calculate inhibitory concentration and the conclusion of activity was presented in (Table 18a, b, Rajendran et al. [38]).

Meenakshisundaram et al. [39] synthesized 3-(4substitutedbenzyl)-6,7-disubstituted-2-(4-(6,7-disubstituted-3-(4-substitutedbenzyl) imidazo[1,2-a] pyridin2-yl)phenyl)imidazo[1,2-a]pyridine, $\quad 3$-(4-substituted benzyl)-2-(3-(6,7-disubstituted-3-(4-substitutedbenzyl) imidazo[1,2-a]pyridin-2-yl)phenyl)-6,7-disubstitutedimidazo[1,2a]pyridine and 6,7-disubstituted-3-(4-substitutedbenzyl)2-phenylimidazo[1,2-a] pyridine (Scheme 19a-c) and evaluated for antitumor potential against different cell lines such as HeLa, MDA-MB-231 and ACHN by SRB method using adriamycin as a reference drug. The conclusion of antitumor potential was presented in (Table 19, Meenakshisundaram et al. [39]).

Sharma et al. [40] synthesized 1,2-disubstituted-4, 5-diphenyl-1H-imidazole (Scheme 20), and evaluated for antitumor potential by using the tryphan blue dye exclusion technique against different cancer cell lines such as DLA and EAC at different concentration. The conclusion of antitumor potential was presented in (Table 20, Sharma et al. [40]).

\section{Antioxidant activity}

Naureen et al. [41] synthesized 3-(4,5-diphenyl-1(substituted phenyl)-1H-imidazol-2-yl)-substituted-2(substituted phenyl)-1H-indole (Scheme 21) and evaluated for antioxidant potential by DPPH method using Quercetin as reference drug. Compound 61d shows the highest antioxidant activity as compared to others. The conclusion of antioxidant potential was presented in (Table 21, Naureen et al. [41]).

Rajasekaran et al. [42] synthesized (E)-(1H-benzo[d] imidazol-1-yl)(4-((substituted benzylidene)amino) phenyl)methanone (Scheme 22a), 2-(1H-benzo[d] 


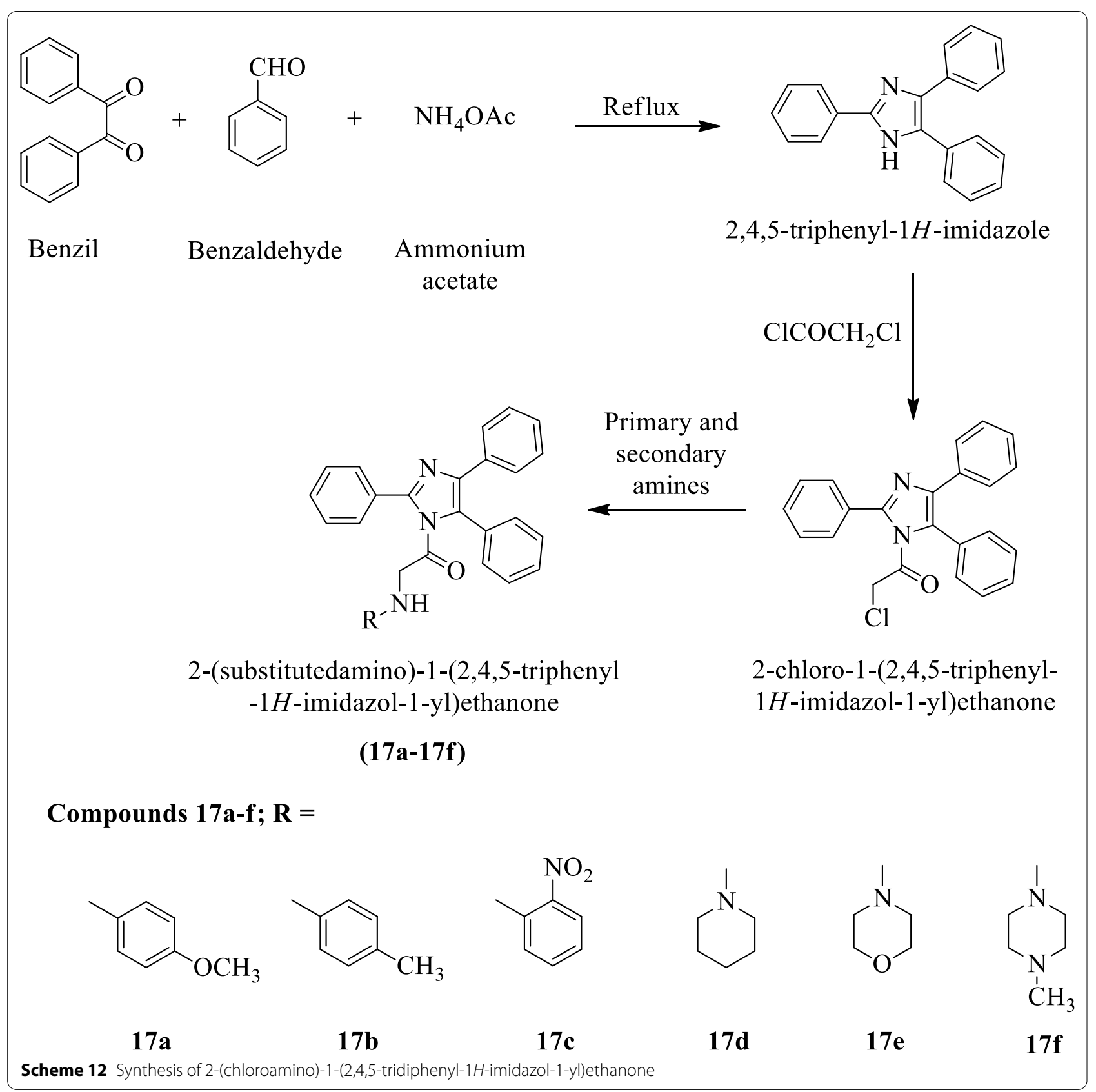


Table 12 Antimicrobial activity of titled compounds (17a-f) Zala et al. [8]

\begin{tabular}{|c|c|c|c|c|}
\hline \multirow[t]{3}{*}{ Compounds } & \multirow{3}{*}{$\begin{array}{l}\text { Concentration } \\
(\mu \mathrm{g} / \mathrm{mL})\end{array}$} & \multicolumn{3}{|c|}{ Zone of inhibition (mm) } \\
\hline & & Gram positive & Gram negative & Fungi \\
\hline & & S. aureus & E. coli & C. albicans \\
\hline \multirow[t]{3}{*}{$17 a$} & 750 & 9 & 10 & 9 \\
\hline & 500 & 8 & 9 & 7 \\
\hline & 250 & 5 & 6 & 5 \\
\hline \multirow[t]{3}{*}{$17 b$} & 750 & 16 & 15 & 15 \\
\hline & 500 & 12 & 11 & 11 \\
\hline & 250 & 10 & 8 & 9 \\
\hline \multirow[t]{3}{*}{$17 c$} & 750 & 26 & 25 & 21 \\
\hline & 500 & 24 & 23 & 19 \\
\hline & 250 & 20 & 19 & 18 \\
\hline \multirow[t]{3}{*}{$17 d$} & 750 & 15 & 16 & 17 \\
\hline & 500 & 13 & 14 & 15 \\
\hline & 250 & 11 & 10 & 12 \\
\hline \multirow[t]{3}{*}{$17 e$} & 750 & 17 & 13 & 19 \\
\hline & 500 & 14 & 11 & 13 \\
\hline & 250 & 12 & 9 & 10 \\
\hline \multirow[t]{3}{*}{$17 f$} & 750 & 9 & 10 & 15 \\
\hline & 500 & 7 & 8 & 13 \\
\hline & 250 & 5 & 6 & 10 \\
\hline \multirow[t]{3}{*}{ Ciprofloxacin } & 750 & 27 & 28 & - \\
\hline & 500 & 26 & 27 & - \\
\hline & 250 & 24 & 25 & - \\
\hline \multirow[t]{3}{*}{ Clotrimazole } & 750 & - & - & 22 \\
\hline & 500 & - & - & 20 \\
\hline & 250 & - & - & 19 \\
\hline
\end{tabular}

imidazol-1-yl)-N-(5-phenyl-1,3,4-oxadiazol-2-yl)acetamide (Scheme 22b) and 1-(1H-benzo[d]imidazol-1-yl)2-((substituted-1,3,4-oxadiazol-2-yl)thio)ethanone (Scheme 22c) and evaluated for antioxidant potential by using DPPH assay. All the synthesized derivatives showed good scavenging potential as compared to ascorbic acid (positive control) and the conclusion of activity was presented in (Table 22, Rajasekaran et al. [42]).

Subramaniam et al. [43] synthesized (Z)-3-(2-(5-(3methyl benzylidene)-4-oxo-2-phenyl-4, 5-dihydro-1H- imidazol-1-yl) ethyl)-2-phenyl quinazolin-4(3H)-one derivatives (Scheme 23) and evaluated for antioxidant potential by using DPPH assay. These compounds showed good scavenging potential as compared to ascorbic acid (positive control). The conclusion of scavenging potential was presented in (Table 23, Subramaniam et al. [43]).

Katikireddy et al. [21] developed (E)-N'-(7-methyl2-propyl-1H-benzo[d]imidazole-5-carbonyl) substituted formohydrazonoyl (Scheme 24) and evaluated for antioxidant activity using ascorbic acid as a reference drug. 


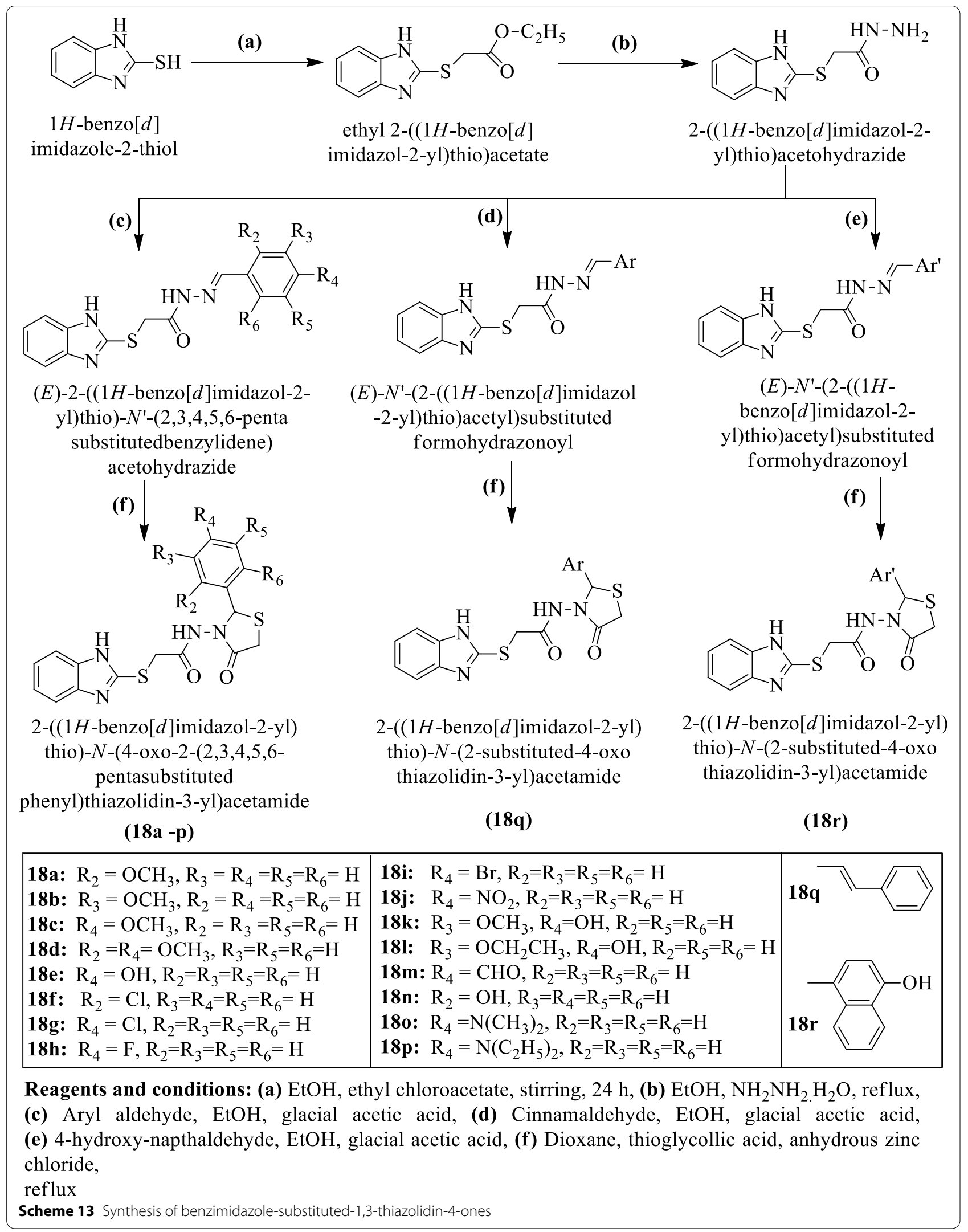


Table 13 MIC of benzimidazole-substituted-1,3-thiazolidin4-ones (18a-r) in $\mu \mathrm{M} / \mathrm{ml}$ Yadav et al. [34]

\begin{tabular}{|c|c|c|c|c|c|}
\hline \multirow[t]{2}{*}{ Compounds } & \multicolumn{5}{|c|}{$\mathrm{MIC}(\mu \mathrm{M} / \mathrm{ml})$} \\
\hline & S. aureus & B. subtilis & E. coli & C. albicans & A. niger \\
\hline $18 a$ & 0.030 & 0.030 & 0.030 & 0.060 & 0.030 \\
\hline $18 b$ & 0.060 & 0.030 & 0.030 & 0.030 & 0.030 \\
\hline $18 \mathrm{C}$ & 0.030 & 0.030 & 0.030 & 0.030 & 0.030 \\
\hline $18 d$ & 0.028 & 0.014 & 0.028 & 0.028 & 0.028 \\
\hline $18 \mathrm{e}$ & 0.031 & 0.031 & 0.031 & 0.031 & 0.031 \\
\hline $18 f$ & 0.030 & 0.030 & 0.030 & 0.030 & 0.030 \\
\hline $18 \mathrm{~g}$ & 0.030 & 0.015 & 0.015 & 0.030 & 0.030 \\
\hline $18 \mathrm{~h}$ & 0.031 & 0.031 & 0.031 & 0.031 & 0.031 \\
\hline $18 \mathrm{i}$ & 0.027 & 0.027 & 0.013 & 0.027 & 0.027 \\
\hline $18 j$ & 0.029 & 0.029 & 0.015 & 0.007 & 0.029 \\
\hline $18 \mathrm{k}$ & 0.058 & 0.029 & 0.007 & 0.029 & 0.029 \\
\hline 181 & 0.028 & 0.028 & 0.028 & 0.028 & 0.028 \\
\hline $18 \mathrm{~m}$ & 0.061 & 0.030 & 0.030 & 0.030 & 0.030 \\
\hline $18 n$ & 0.031 & 0.031 & 0.008 & 0.031 & 0.031 \\
\hline 180 & 0.029 & 0.029 & 0.029 & 0.029 & 0.029 \\
\hline $18 p$ & 0.027 & 0.027 & 0.027 & 0.027 & 0.027 \\
\hline $18 q$ & 0.030 & 0.030 & 0.030 & 0.030 & 0.030 \\
\hline $18 r$ & 0.028 & 0.028 & 0.028 & 0.028 & 0.028 \\
\hline Norfloxacin & 0.47 & 0.47 & 0.47 & - & - \\
\hline Fluconazole & - & - & - & 0.50 & 0.50 \\
\hline
\end{tabular}

Compound 64n shows the most potent antioxidant activity as compared to others and the results of activity were presented in (Table 24, Katikireddy et al. [21]).

Subhashini et al. [44] synthesized 4-((4-(4,5-diphenyl1H-imidazol-2-yl)phenoxy)methyl)-1-(2,3,4-trisubstituted phenyl)-1H-1,2,3-triazole derivatives (Scheme 25a, b) and evaluated for antioxidant activity by using four different methods such as Hydrogen peroxide scavenging, Nitric oxide scavenging, DPPH, and FRAP assay. The conclusion of antioxidant potential was presented in (Table 25a-d, Subhashini et al. [44]).

\section{Antihypertensive activity}

Navarrete-Vazquez et al. [45] synthesized 5-(trifluoromethyl)2-(2,3,4-trisubstituted phenyl)-1H-benzo[d] imidazole and 5- nitro-2-(2,3,4-trisubstituted phenyl)-1H-benzo [d] Imidazole (Scheme 26) and evaluated for antihypertensive potential in SHR by using the tail-cuff method and the results of antihypertensive activity were summarized in (Table 26, NavarreteVazquez et al. [45]).

Hadizadeh et al. [46] synthesized 2-(2-(1H-imidazol-1-yl) ethyl)-4-(1-benzyl-2-(substituted thio)-1H-imidazol-5-yl)5-(substituted carbonyl)-6-methyl-1, 4-dihydropyridine3-substituted carboxylic acid (Scheme 27) and evaluated for antihypertensive potential in rats and the results of antihypertensive activity were summarized in (Table 27, Hadizadeh et al. [46]).

Goyal et al. [22] synthesized 2-substituted-1-(pyridin-2ylmethyl)-1H-benzo[d]imidazole derivatives (Scheme 28) and evaluated for antihypertensive potential and the 


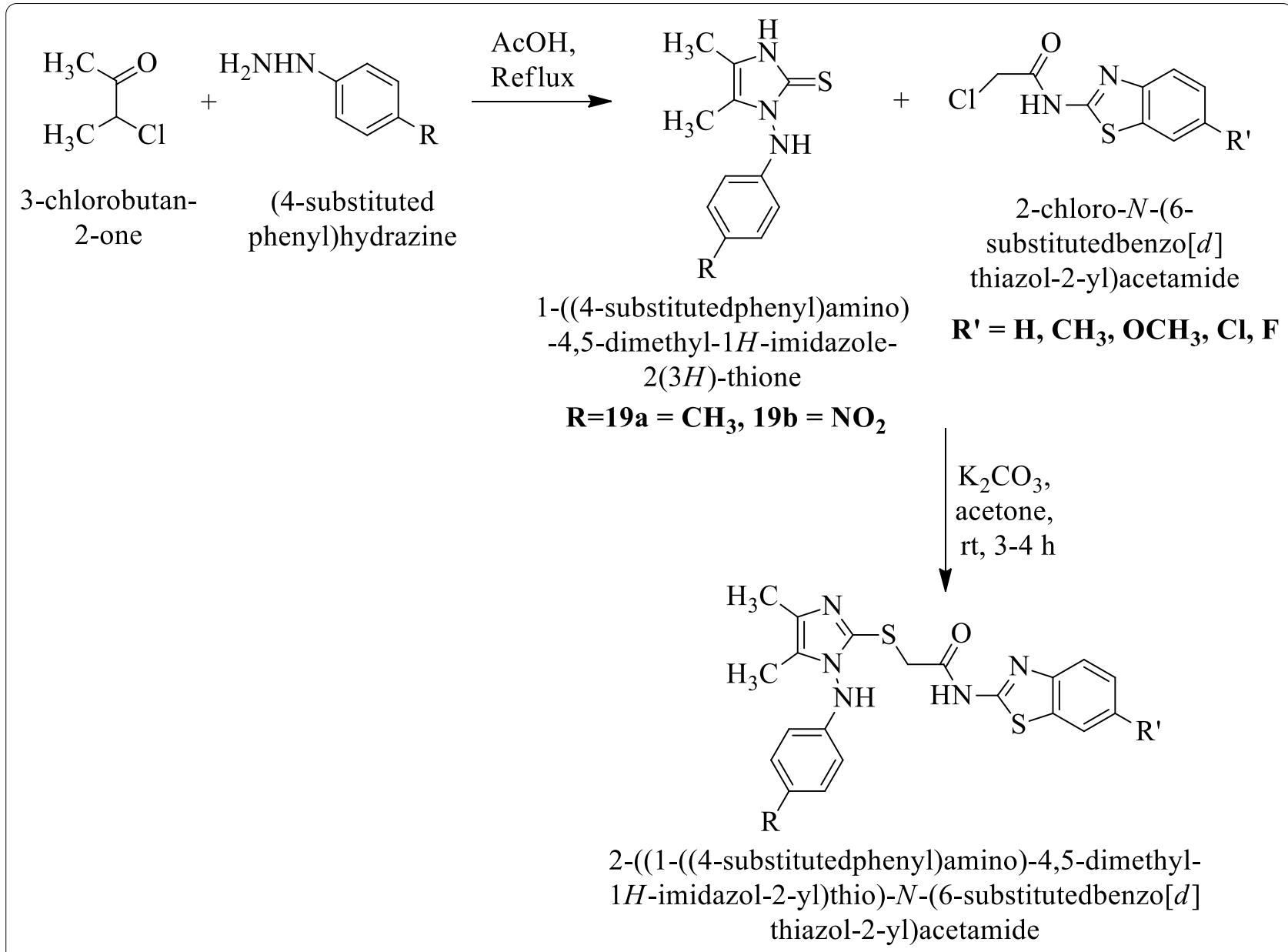

(20a-j)

Scheme 14 Synthesis of 2-((1-((4-substitutedphenyl)amino)-4,5-dimethyl-1H-imidazol-2-yl)thio)-N-(6-substitutedbenzo[d]thiazol-2-yl)acetamide

Table 14 IC $_{50}$ values of the synthesized compounds (20a-j) against $\mathrm{C} 6$ and HepG2 cancer cell line Yurttas et al. [35]

\begin{tabular}{lll}
\hline Compounds & \multicolumn{2}{l}{$\mathbf{C}_{\mathbf{5 0}}$ value } \\
\cline { 2 - 3 } & $\mathbf{C 6}$ & HepG2 \\
\hline $20 \mathrm{a}$ & $27.0 \pm 1.41$ & $50.0 \pm 5.0$ \\
$20 \mathrm{~b}$ & $20 \pm 2.0$ & $26.33 \pm 1.53$ \\
$20 \mathrm{c}$ & $32.67 \pm 6.43$ & $275.0 \pm 35.36$ \\
$20 \mathrm{~d}$ & $22.0 \pm 3.61$ & $29.33 \pm 1.15$ \\
$20 \mathrm{e}$ & $16.33 \pm 2.31$ & $31.67 \pm 7.23$ \\
$20 f$ & $19.50 \pm 2.12$ & $28.67 \pm 1.15$ \\
$20 \mathrm{~g}$ & $15.67 \pm 2.52$ & $58.33 \pm 2.89$ \\
$20 \mathrm{~h}$ & $>500$ & $>500$ \\
$20 \mathrm{i}$ & $24.33 \pm 4.04$ & $>500$ \\
$20 \mathrm{j}$ & $19.33 \pm 2.31$ & $>500$ \\
Cisplatin & $23.0 \pm 1.73$ & $46.67 \pm 7.64$ \\
\hline
\end{tabular}

results of activity were summarized in (Table 28, Goyal et al. [22]).

\section{Antitubercular activity}

Amini et al. [47] synthesized N3-(substituted phenyl)N5-(substituted phenyl)-4-(4,5-dichloro-1H-imidazol2-yl)-2-methyl-1, 4-dihydropyridine-3,5-dicarboxamide (Scheme 29) and evaluated for anti-tubercular activity against Mycobacterium tuberculosis strain using rifampicin as reference drug. The conclusion of the antitubercular activity was presented in (Table 29, Amini et al. [47]). 
<smiles>Nc1ccccc1N</smiles>

benzene-1,2diamine (i)<smiles>CC(O)c1nc2ccccc2[nH]1</smiles>

1-(1H-benzo[d] imidazol-2-yl)ethanol (ii)<smiles>CC(=O)c1nc2ccccc2[nH]1</smiles>

1-(1H-benzo $[d]$ imidazol-2-yl)ethanone (iii)<smiles>[R1]c1ccc(/C=C/C(=O)c2nc3ccccc3[nH]2)cc1</smiles>

(E)-1-(1H-benzo[ $d]$ imidazol-2-yl)-3-(4substitutedphenyl)prop-2-en-1-one

21a: $R_{1}=H$

21b: $\mathrm{R}_{1}=\mathrm{CH}_{3}$

21c: $\mathrm{R}_{1}=\mathrm{OCH}_{3}$

21d: $\mathrm{R}_{1}=\mathrm{Cl}$ $\checkmark$<smiles>[R]c1ccc(/C=C/C(=O)c2nc3ccccc3n2CC=C)cc1</smiles>

22a: $\mathrm{R}_{1}=\mathrm{H}$

22b: $\mathrm{R}_{1}=\mathrm{CH}_{3}$

22c: $\mathrm{R}_{1}=\mathrm{OCH}_{3}$

22d: $\mathrm{R}_{1}=\mathrm{Cl}$

(22a-d)<smiles>[3H]C1CCN(CCCl)C1</smiles><smiles>[R]c1ccc(/C=C/C(=O)c2nc3ccccc3n2CCN2CCCC2)cc1</smiles>

23a: $\mathrm{R}_{1}=\mathrm{H}$

23b: $\mathrm{R}_{1}=\mathrm{CH}_{3}$

23c: $\mathrm{R}_{1}=\mathrm{OCH}_{3}$

23d: $\mathrm{R}_{1}=\mathrm{Cl}$<smiles>[Y]C1CCN(CCCl)CC1</smiles><smiles>[Y]C1COCCN1CCCl</smiles><smiles>ClCCCN1CCOCC1</smiles><smiles>[R]c1ccc(/C=C/C(=O)c2nc3ccccc3n2CCN2CCCCC2)cc1</smiles><smiles>[R]c1ccc(/C=C/C(=O)c2nc3ccccc3n2CCN2CCOCC2)cc1</smiles>

25a: $\mathrm{R}_{1}=\mathrm{H}$ 25b: $\mathrm{R}_{1}=\mathrm{CH}_{3}$ 25c: $\mathrm{R}_{1}=\mathrm{OCH}_{3}$ 25d: $\mathrm{R}_{1}=\mathrm{Cl}$

(25a -d)

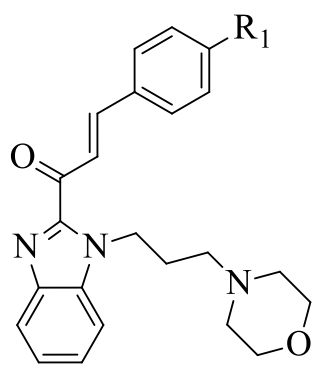

24a: $\mathrm{R}_{1}=\mathrm{H}$

24b: $\mathrm{R}_{1}=\mathrm{CH}_{3}$

24c: $\mathrm{R}_{1}=\mathrm{OCH}_{3}$

24d: $\mathrm{R}_{1}=\mathrm{Cl}$

(24a-d) 26a: $R_{1}=H$

26b: $\mathrm{R}_{1}=\mathrm{CH}_{3}$ 26c: $\mathrm{R}_{1}=\mathrm{OCH}_{3}$ 26d: $\mathrm{R}_{1}=\mathrm{Cl}$

Reagents and conditions : (i) Lactic acid, $4 \mathrm{~N} \mathrm{HCl}$, Reflux (ii) permanganate, solid aluminium oxide, no solvent, r.t, $10 \mathrm{~min}$, (iii) Benzaldehyde, $40 \% \mathrm{KOH}$, EtOH, r.t, $10 \mathrm{~min}$, (iv) potassium carbonateacetonitrile, reflux overnight

Scheme 15 Synthesis of imidazole derivatives 
Table 15 Anticancer activity of titled compounds (21a-26d) against different cancer cell lines Hsieh et al. [25]

\begin{tabular}{|c|c|c|c|c|}
\hline \multirow[t]{2}{*}{ Compounds } & \multicolumn{4}{|c|}{ Cancer cells $\left(\mathrm{IC}_{50} \mu \mathrm{M}\right)$} \\
\hline & A549 & MCF-7 & HEP-G2 & OVCAR-3 \\
\hline $21 a$ & $119.3 \pm 29.9$ & $13.49 \pm 0.16$ & $24.2 \pm 0.32$ & $16.91 \pm 0.37$ \\
\hline $21 b$ & $19.17 \pm 0.43$ & $18.09 \pm 0.28$ & $59.13 \pm 0.92$ & $24.7 \pm 1.69$ \\
\hline $21 c$ & $17.41 \pm 0.16$ & $16.04 \pm 0.24$ & $140.85 \pm 0.88$ & $34.44 \pm 1.55$ \\
\hline $21 d$ & $35.89 \pm 0.84$ & $32.55 \pm 3.26$ & $36.54 \pm 1.35$ & $36.48 \pm 1.36$ \\
\hline $22 a$ & $12.47 \pm 0.18$ & $12.12 \pm 0.10$ & $15.44 \pm 0.25$ & $16.09 \pm 0.39$ \\
\hline $22 b$ & $41.05 \pm 1.61$ & $53.54 \pm 1.12$ & $117.28 \pm 2.42$ & $59.01 \pm 8.91$ \\
\hline $22 \mathrm{C}$ & $>314$ & $254.9 \pm 13.6$ & $>314$ & $299.52 \pm 9.27$ \\
\hline $22 d$ & $15.79 \pm 0.49$ & $13.42 \pm 0.24$ & $17.6 \pm 0.25$ & $16.13 \pm 0.32$ \\
\hline $23 a$ & $10.3 \pm 0.13$ & $9.65 \pm 0.06$ & $10.16 \pm 0.08$ & $10.5 \pm 0.10$ \\
\hline $23 b$ & $54.12 \pm 1.20$ & $53.19 \pm 0.77$ & $64.91 \pm 0.24$ & $28.71 \pm 1.44$ \\
\hline $23 c$ & $56.21 \pm 0.96$ & $56.09 \pm 0.14$ & $36.61 \pm 1.89$ & $11.4 \pm 0.24$ \\
\hline $23 d$ & $19.53 \pm 0.71$ & $14.73 \pm 0.09$ & $15.49 \pm 0.16$ & $14.04 \pm 0.29$ \\
\hline $24 a$ & $10.73 \pm 0.58$ & $9.73 \pm 0.16$ & $10.33 \pm 0.06$ & $10.34 \pm 0.19$ \\
\hline $24 b$ & $11.64 \pm 0.25$ & $11.14 \pm 0.07$ & $32.16 \pm 1.83$ & $12.55 \pm 0.12$ \\
\hline $24 c$ & $22.36 \pm 0.54$ & $21.12 \pm 0.53$ & $58.74 \pm 0.75$ & $13.29 \pm 0.47$ \\
\hline $24 d$ & $50.45 \pm 0.82$ & $54.41 \pm 0.72$ & $56.45 \pm 0.86$ & $33.13 \pm 0.14$ \\
\hline $25 a$ & $14.59 \pm 0.40$ & $10.38 \pm 0.08$ & $36.13 \pm 0.75$ & $22.44 \pm 0.47$ \\
\hline $25 b$ & $10.76 \pm 0.29$ & $10.15 \pm 0.06$ & $42.05 \pm 0.91$ & $16.32 \pm 0.45$ \\
\hline $25 c$ & $10.27 \pm 0.15$ & $11.12 \pm 0.20$ & $50.24 \pm 0.88$ & $14.88 \pm 0.67$ \\
\hline $25 d$ & $24.06 \pm 0.08$ & $22.93 \pm 0.49$ & $21.38 \pm 0.68$ & $0.14 .22 \pm 0.33$ \\
\hline $26 a$ & $9.73 \pm 0.07$ & $8.91 \pm 0.07$ & $10.93 \pm 0.10$ & $10.76 \pm 0.12$ \\
\hline $26 b$ & $11.79 \pm 0.27$ & $11.34 \pm 0.17$ & $47.88 \pm 0.76$ & $13.76 \pm 0.27$ \\
\hline $26 c$ & $16.92 \pm 0.61$ & $11.93 \pm 0.14$ & $32.92 \pm 0.38$ & $13.4 \pm 0.33$ \\
\hline $26 d$ & $81.48 \pm 1.40$ & $35.69 \pm 0.47$ & $95.7 \pm 2.44$ & $42.24 \pm 2.43$ \\
\hline DOX & $0.46 \pm 0.01$ & $0.42 \pm 0.01$ & $0.72 \pm 0.01$ & $3.95 \pm 0.09$ \\
\hline Cisplatin & $7.31 \pm 0.44$ & $11.7 \pm 0.12$ & $3.97 \pm 0.04$ & $16.04 \pm 0.74$ \\
\hline
\end{tabular}

Pandey et al. [48] synthesized (E)-3-(4-(7-substituted3-(substituted amino)imidazo[1,2-a] pyridin-2-yl)phenyl)1-(substituted phenyl)prop-2-en-1-one (Scheme 30) and evaluated for anti-tubercular potential against Mycobacterium tuberculosis strain by $\mathbf{M B} 7 \mathbf{H 1 0}$ agar medium using Ethambutol and Pyrazinamide as a reference drug. The conclusion of the activity was presented in (Table 30, Pandey et al. [48]).

Makwane et al. [49] synthesized 10-(2-(substituted phenyl)imidazo[2,1-b][1,3,4] thiadiazol-6-yl)-10H-phenothiazine by using (Scheme 31) and evaluated for antitubercular activity by (L.J) agar method against Mycobacterium tuberculosis $\mathbf{H}_{37} \mathbf{R v}$ strain using Isoniazid as reference drug and MIC values of these derivatives were calculated. The conclusion of anti-tubercular activity was presented in (Table 31, Makwane et al. [49]).

Nandha et al. [23] synthesized 2-((1H-imidazol-1-yl $)$ methyl)-6-substituted-5-fluoro-1H-benzo[d]imidazole (Scheme 32) and evaluated for anti-tubercular activity against Mycobacterium tuberculosis strain by MABA assay using Isoniazid as a reference drug. The 


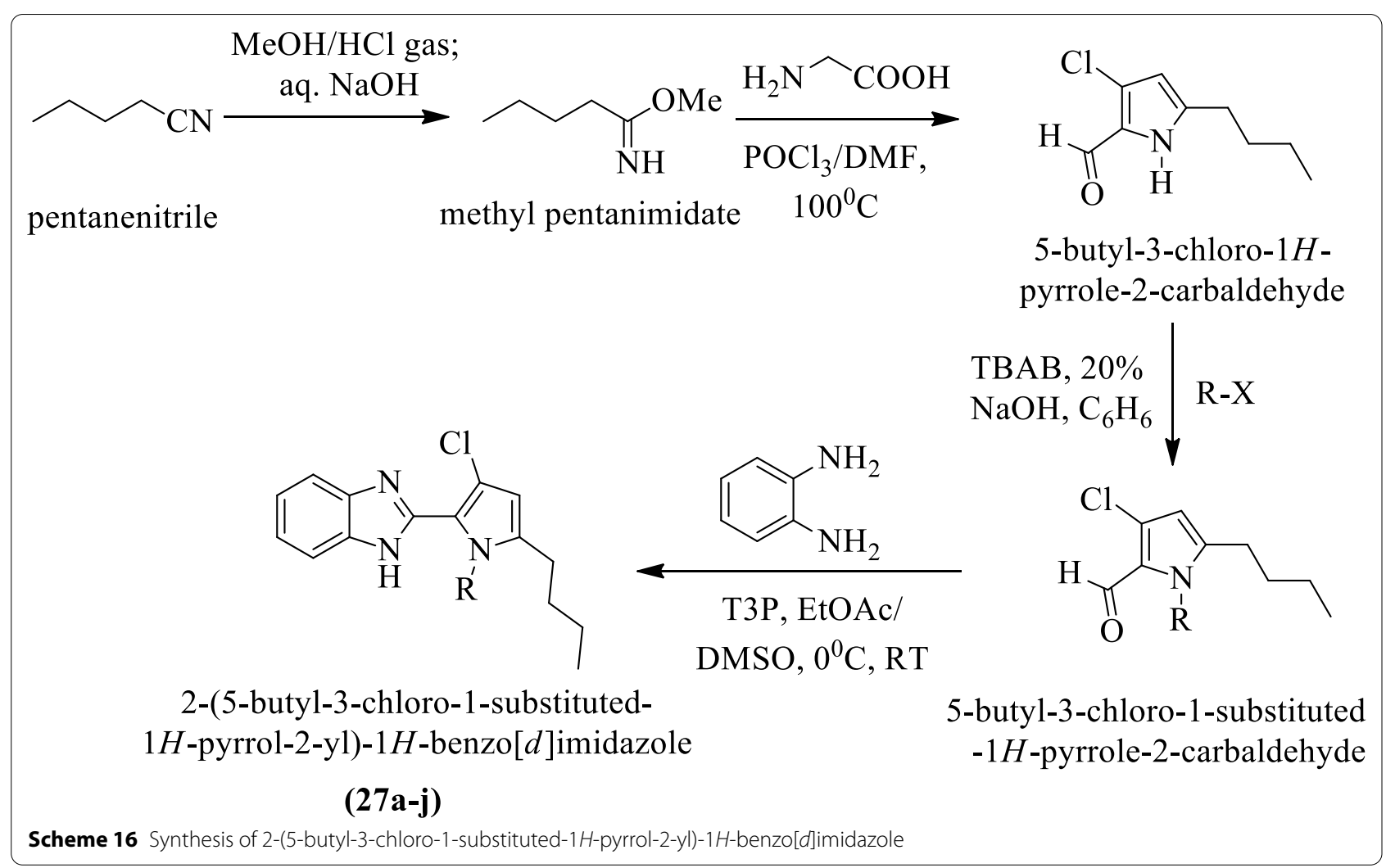

Table $16 \mathrm{IC}_{50}$ values of the synthesized compounds (27a-j) Roopashree et al. [36]

\begin{tabular}{llll}
\hline Compounds & $\mathbf{R}-\mathbf{X}-\mathbf{5}$ & $\mathbf{R}(\mathbf{6})$ & $\mathbf{I C} \mathbf{5 0}(\boldsymbol{\mu M}) \pm \mathbf{S D}$ \\
\hline $27 \mathrm{a}$ & $\mathrm{CH}_{3} \mathrm{l}$ & $\mathrm{CH}_{3}$ & $>50$ \\
$27 \mathrm{~b}$ & $\mathrm{EtBr}$ & $\mathrm{Et}$ & $>50$ \\
$27 \mathrm{C}$ & $\mathrm{CH}_{3}\left(\mathrm{CH}_{2}\right)_{2} \mathrm{CH}_{2} \mathrm{Br}$ & $\mathrm{CH}_{3}\left(\mathrm{CH}_{2}\right)_{2} \mathrm{CH}_{2}$ & $>50$ \\
$27 \mathrm{~d}$ & $\mathrm{CH}_{3}\left(\mathrm{CH}_{2}\right)_{5} \mathrm{CH}_{2} \mathrm{Br}$ & $\mathrm{CH}_{3}\left(\mathrm{CH}_{2}\right)_{5} \mathrm{CH}_{2}$ & $25.3 \pm 4.18$ \\
$27 \mathrm{e}$ & $3-\mathrm{MeC}_{6} \mathrm{H}_{4} \mathrm{CH}_{2} \mathrm{Br}$ & $3-\mathrm{MeC}_{6} \mathrm{H}_{4} \mathrm{CH}_{2}$ & $30.2 \pm 2.27$ \\
$27 f$ & $3-\mathrm{MeOC}_{6} \mathrm{H}_{4} \mathrm{CH}_{2} \mathrm{Br}$ & $3-\mathrm{MeOC}_{6} \mathrm{H}_{4} \mathrm{CH}_{2}$ & $>50$ \\
$27 \mathrm{~g}$ & $4-\mathrm{ClC}_{6} \mathrm{H}_{4} \mathrm{CH}_{2} \mathrm{Br}$ & $4-\mathrm{ClC}_{6} \mathrm{H}_{4} \mathrm{CH}_{2}$ & $>50$ \\
$27 \mathrm{~h}$ & $3,4-\mathrm{Cl}_{2} \mathrm{C}_{6} \mathrm{H}_{3} \mathrm{CH}_{2} \mathrm{Br}$ & $3,4-\mathrm{Cl}_{2} \mathrm{C}_{6} \mathrm{H}_{3} \mathrm{CH}_{2}$ & $31.9 \pm 4.77$ \\
$27 \mathrm{i}$ & $4-\mathrm{FC}_{6} \mathrm{H}_{4} \mathrm{CH}_{2} \mathrm{Br}$ & $4-\mathrm{FC}_{6} \mathrm{H}_{4} \mathrm{CH}_{2}$ & $30.0 \pm 5.12$ \\
$27 \mathrm{j}$ & $\mathrm{CH}_{6} \mathrm{H}_{5} \mathrm{CH}_{2} \mathrm{Br}$ & $\mathrm{C}_{6} \mathrm{H}_{5} \mathrm{CH}_{2}$ & $>50$ \\
Sorafenib & & & $4.1 \pm 0.9$ \\
\hline
\end{tabular}

SD Standard deviation, $I C_{50}$ Inhibitory concentration $50 \%$ 


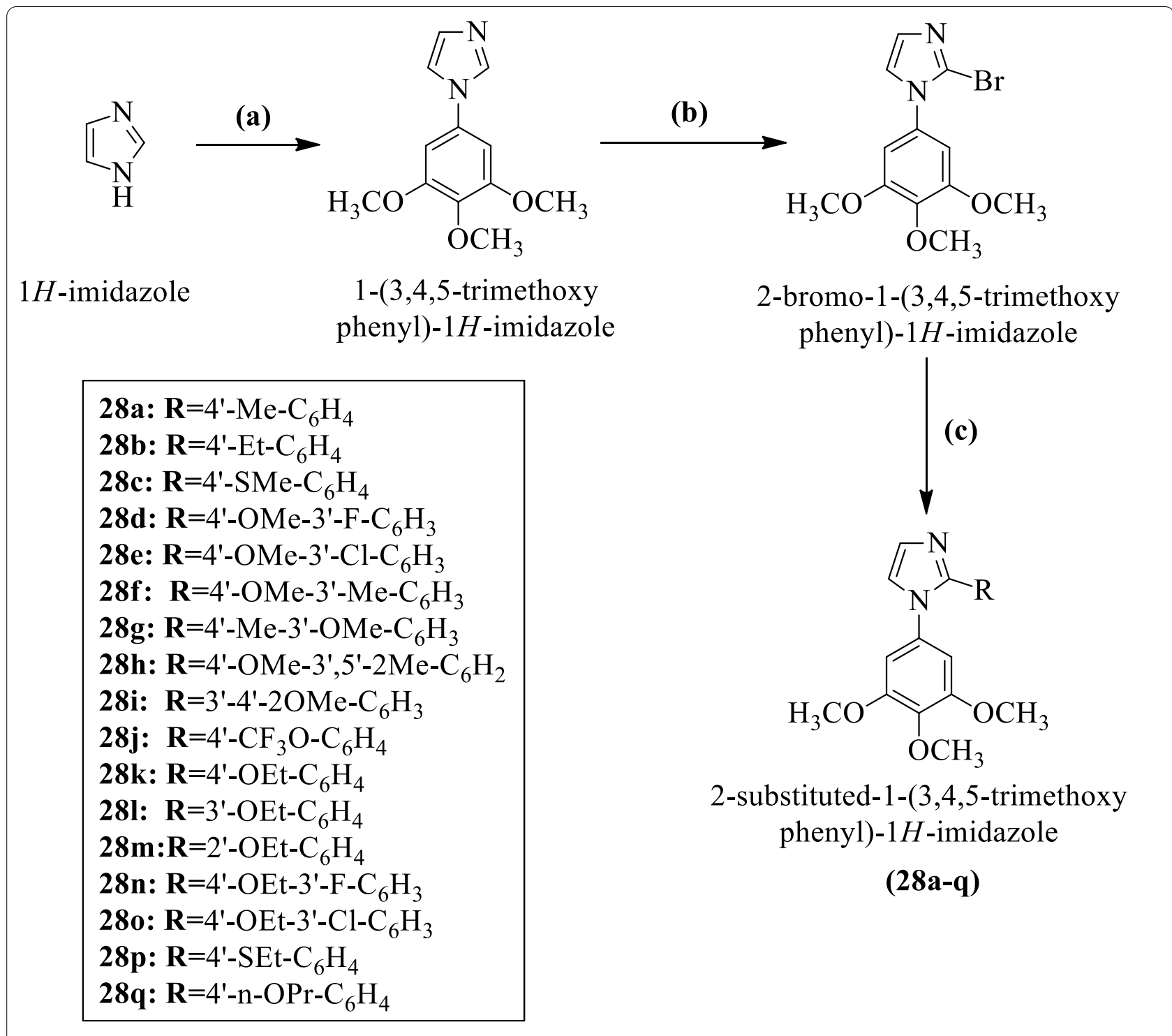

Reagents and conditions: (a) 1-bromo-3,4,5-trimetyhoxybenzene, $\mathrm{Cs}_{2} \mathrm{CO}_{3}, \mathrm{CuI}, \mathrm{DMF}$, $120^{\circ} \mathrm{C}, 40 \mathrm{~h}$ (b) NBS, $\mathrm{CH}_{3} \mathrm{CN}$ (c) $\mathrm{PdCl}_{2}$ (DPPF), $\mathrm{ArB}(\mathrm{OH})_{2}, \mathrm{CsF}, 1,4$-dioxane, $65^{\circ} \mathrm{C}$ 
Table 17 Antitumor activity of the synthesized compounds (28a-q) Romangoli et al. [37]

\begin{tabular}{|c|c|c|c|c|c|c|c|}
\hline \multirow[t]{2}{*}{ Compounds } & \multicolumn{7}{|l|}{$I C_{50}(\mu \mathrm{M})$} \\
\hline & HeLa & HT-29 & A549 & MCF-7 & Jurkat & RS4-11 & HL-60 \\
\hline $28 a$ & $1260 \pm 172$ & $1915 \pm 354$ & $4733 \pm 328$ & $2800 \pm 721$ & $760 \pm 136$ & $>10,000$ & $2100 \pm 252$ \\
\hline $28 b$ & $1985 \pm 126$ & $1400 \pm 200$ & $7000 \pm 1153$ & $2090 \pm 374$ & $7569 \pm 758$ & $5678 \pm 259$ & $4800 \pm 451$ \\
\hline $28 c$ & $337 \pm 48$ & $330 \pm 36$ & $5600 \pm 352$ & $1363 \pm 349.8$ & $407 \pm 24$ & $800 \pm 58$ & $333 \pm 41$ \\
\hline $28 d$ & $51 \pm 6.5$ & $112 \pm 15$ & $121 \pm 56$ & $74 \pm 17$ & $90 \pm 23$ & $217 \pm 46$ & $29 \pm 9.5$ \\
\hline $28 e$ & $263 \pm 39$ & $647 \pm 83$ & $2600 \pm 422$ & $666 \pm 231$ & $365 \pm 25$ & $715 \pm 148$ & $453 \pm 14$ \\
\hline $28 f$ & $330 \pm 25$ & $377 \pm 83$ & $4717 \pm 509$ & $509 \pm 25$ & $136 \pm 38$ & $475 \pm 106$ & $413 \pm 27$ \\
\hline $28 \mathrm{~g}$ & $623 \pm 98$ & $>10,000$ & $>10,000$ & $>10,000$ & $4933 \pm 536$ & $2567 \pm 784$ & $>10,000$ \\
\hline $28 \mathrm{~h}$ & $9157 \pm 1593$ & $>10,000$ & $>10,000$ & $>10,000$ & $>10,000$ & $>10,000$ & $3466 \pm 467$ \\
\hline $28 \mathrm{i}$ & $>10,000$ & $>10,000$ & $>10,000$ & $>10,000$ & $>10,000$ & $>10,000$ & $3933 \pm 517$ \\
\hline $28 j$ & $>10,000$ & $>10,000$ & $>10,000$ & $>10,000$ & $6633 \pm 338$ & $>10,000$ & $>10,000$ \\
\hline $28 k$ & $3.7 \pm 0.12$ & $1.8 \pm 0.8$ & $1.9 \pm 1.0$ & $1.5 \pm 0.2$ & $1.2 \pm 0.5$ & $34.7 \pm 0.0$ & $4.8 \pm 1.9$ \\
\hline 281 & $>10,000$ & $>10,000$ & $>10,000$ & $>10,000$ & $>10,000$ & $>10,000$ & $>10,000$ \\
\hline $28 \mathrm{~m}$ & $>10,000$ & $>10,000$ & $>10,000$ & $>10,000$ & $>10,000$ & $>10,000$ & $>10,000$ \\
\hline $28 n$ & $1.5 \pm 0.32$ & $7.5 \pm 1.2$ & $14 \pm 2.3$ & $3.4 \pm 0.38$ & $12 \pm 6.6$ & $8.6 \pm 1.1$ & $3.5 \pm 0.73$ \\
\hline 280 & $3.8 \pm 0.7$ & $0.4 \pm 0.06$ & $0.57 \pm 0.17$ & $0.7 \pm 0.06$ & $0.9 \pm 0.2$ & $1.2 \pm 0.7$ & $1.8 \pm 0.6$ \\
\hline $28 p$ & $48 \pm 2.5$ & $174 \pm 16$ & $228 \pm 81$ & $69 \pm 7.0$ & $127 \pm 27$ & $85 \pm 20$ & $12 \pm 2.5$ \\
\hline $28 q$ & $2.9 \pm 0.8$ & $15 \pm 1.3$ & $63 \pm 18.1$ & $1.7 \pm 0.6$ & $42 \pm 3.9$ & $91 \pm 8.9$ & $63.0 \pm 17.6$ \\
\hline$C A-4$ & $4 \pm 1$ & $180 \pm 30$ & $3100 \pm 100$ & $5 \pm 0.6$ & $0.8 \pm 0.2$ & $370 \pm 100$ & $1 \pm 0.2$ \\
\hline
\end{tabular}

conclusion of anti-tubercular activity was presented in (Table 32, Nandha et al. [23]).

Nandha et al. [50] synthesized 6-(benzo[d] [1,3]dioxol5-yloxy)-2-substituted-5-fluoro-1H-benzo[d] imidazole (Scheme 33) and evaluated for anti-tubercular activity against Mycobacterium tuberculosis (ATCC27294) by MABA assay using streptomycin, ciprofloxacin, and pyrazinamide as a reference drug. The conclusion of the activity was presented in (Table 33, Nandha et al. [50]).

Gising et al. [51] synthesized 2,5-disubstituted4-(6-methoxynaphthalen-2-yl)-1H-imidazole by using (Scheme 34). The anti-tubercular potential of these derivatives was evaluated against Mycobacterium tuberculosis strain and MIC values of these derivatives were calculated. The conclusion of anti-tubercular activity was presented in (Table 34, Gising et al. [51]).

Syed et al. [52] synthesized 6-(4-substituted phenyl)2-(3,5-dimethyl-1H-pyrazol-1-yl)imidazo [2,1-b][1,3,4] thiadiazole (Scheme 35) and evaluated for anti-tubercular potential against Mycobacterium tuberculosis strain. Compounds 80a, 80b, 81a, 82a, and 83a showed the most potent anti-tubercular activity as compared to others. The conclusion of anti-tubercular activity was presented in (Table 35, Syed et al. [52]). 


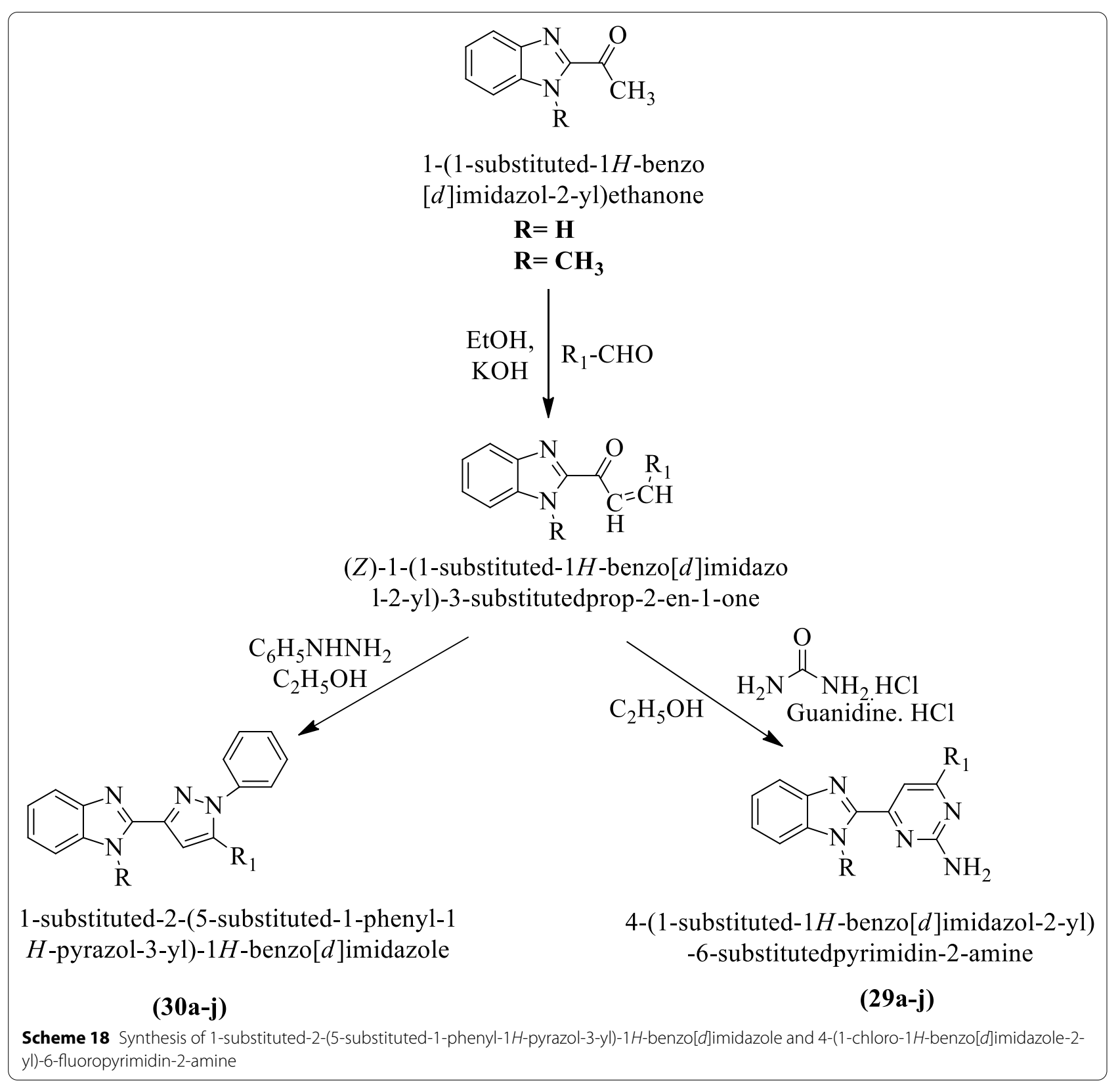


Table 18 (a) IC 50 of the titled compounds (29a-j) against of MCF-7 and CaCo-2 cell line-benzo [d] imidazole pyrimidine derivatives; (b) IC ${ }_{50}$ of the titled compounds (30a-j) against of MCF-7 and CaCo-2 cell line-benzo [d] imidazole pyrazole derivatives Rajendran et al. [38]

\begin{tabular}{|c|c|c|c|c|c|}
\hline \multirow[t]{2}{*}{ Compounds } & \multirow[t]{2}{*}{ Substituent $\mathrm{R}$} & \multirow[t]{2}{*}{ Substituent $R_{1}$} & \multirow[t]{2}{*}{ Molecular formula } & \multicolumn{2}{|c|}{$\mathrm{IC} 50 \pm \mathrm{SD}(\mu \mathrm{M})$} \\
\hline & & & & MCF-7 & CaCo-2 \\
\hline \multicolumn{6}{|l|}{ (a) } \\
\hline $29 a$ & $\mathrm{H}$ & & $\mathrm{C}_{17} \mathrm{H}_{13} \mathrm{~N}_{5}$ & $8.22 \pm 1.48$ & $5.67 \pm 1.25$ \\
\hline $29 b$ & $\mathrm{H}$ & & $\mathrm{C}_{16} \mathrm{H}_{12} \mathrm{~N}_{6}$ & $10.43 \pm 1.45$ & $9.56 \pm 1.33$ \\
\hline $29 c$ & $\mathrm{H}$ & & $\mathrm{C}_{19} \mathrm{H}_{17} \mathrm{~N}_{5} \mathrm{O}_{2}$ & $>30$ & $28.40 \pm 2.48$ \\
\hline $29 d$ & $\mathrm{H}$ & & $\mathrm{C}_{18} \mathrm{H}_{14} \mathrm{ClN}_{5} \mathrm{O}$ & $13.05 \pm 2.07$ & $12.33 \pm 1.80$ \\
\hline $29 e$ & $\mathrm{H}$ & & $\mathrm{C}_{25} \mathrm{H}_{17} \mathrm{~N}_{5}$ & $>30 \pm 2.87$ & $>30 \pm 2.98$ \\
\hline $29 f$ & $\mathrm{CH}_{3}$ & & $\mathrm{C}_{18} \mathrm{H}_{15} \mathrm{~N}_{5}$ & $>30 \pm 2.66$ & $>30 \pm 2.43$ \\
\hline $29 \mathrm{~g}$ & $\mathrm{CH}_{3}$ & & $\mathrm{C}_{17} \mathrm{H}_{14} \mathrm{~N}_{6}$ & $18.56 \pm 2.82$ & $16.23 \pm 1.24$ \\
\hline $29 \mathrm{~h}$ & $\mathrm{CH}_{3}$ & & $\mathrm{C}_{19} \mathrm{H}_{16} \mathrm{ClN}_{5} \mathrm{O}$ & $>30 \pm 2.19$ & $25.50 \pm 2.74$ \\
\hline $29 i$ & $\mathrm{CH}_{3}$ & & $\mathrm{C}_{20} \mathrm{H}_{19} \mathrm{~N}_{5} \mathrm{O}_{2}$ & $25.11 \pm 2.44$ & $21.89 \pm 2.35$ \\
\hline 29j & $\mathrm{CH}_{3}$ & & $\mathrm{C}_{26} \mathrm{H}_{19} \mathrm{~N}_{5}$ & $>30 \pm 2.80$ & $>30 \pm 2.06$ \\
\hline Fluorouracil & & & & $7.26 \pm 2.30$ & $5.23 \pm 2.36$ \\
\hline
\end{tabular}


Table 18 (continued)

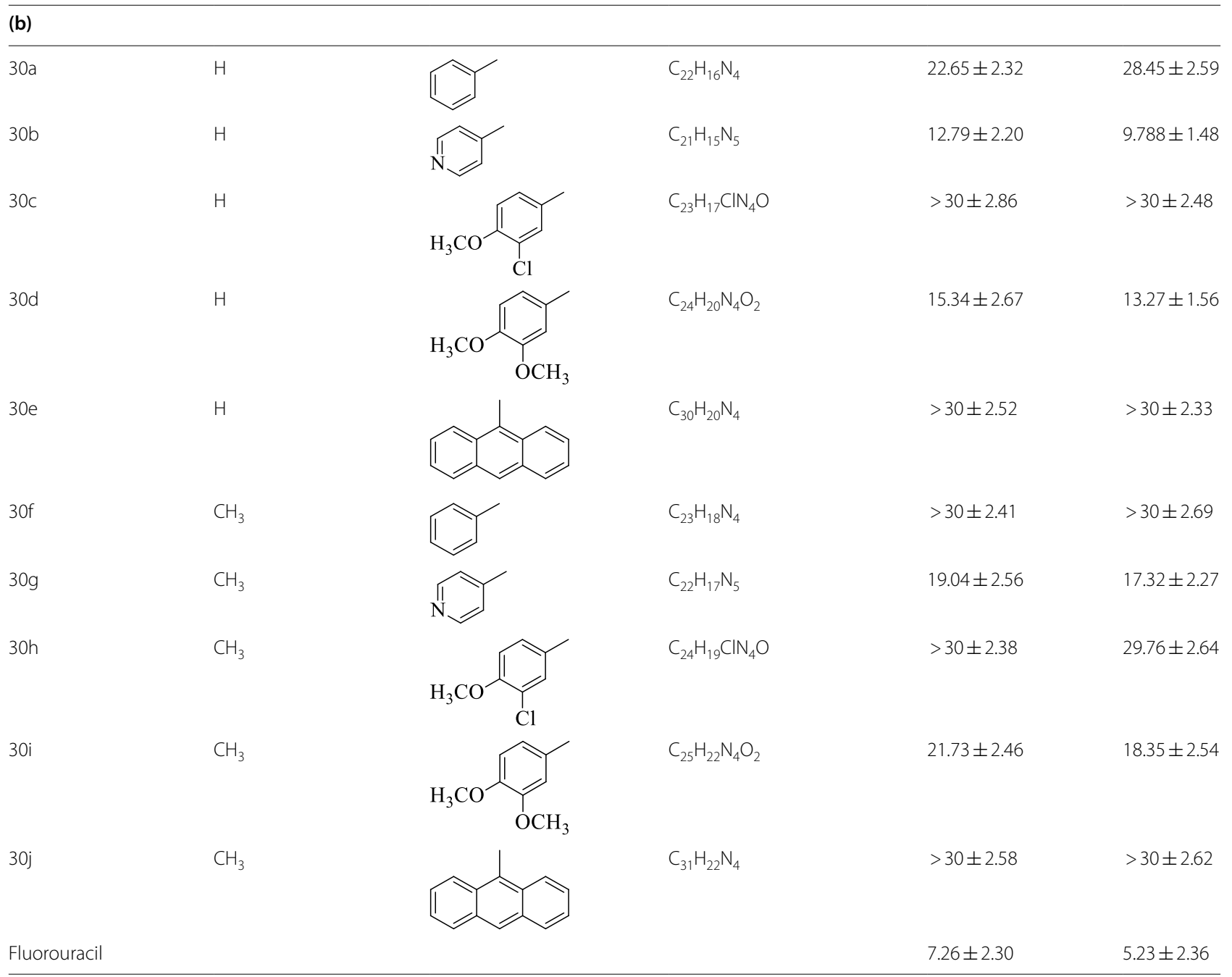




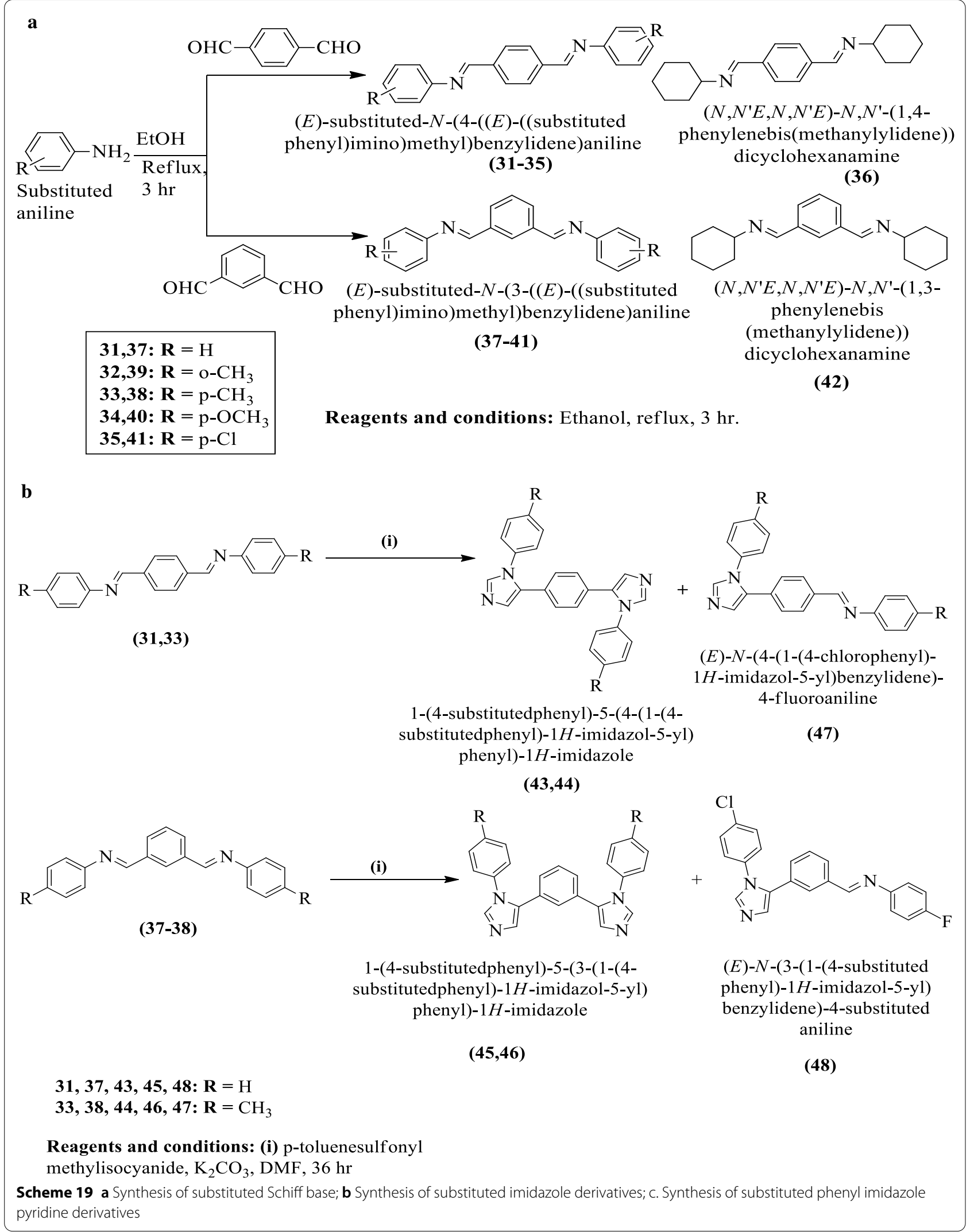




$$
\begin{aligned}
& \text { c } \\
& \overbrace{=\mathrm{N}}^{\mathrm{NH}_{2}}+\mathrm{OHC}-\mathrm{CHO} \\
& \text { (i) } \\
& \begin{array}{l}
\text { 4,5-disubstituted } \\
\text { pyridin-2-amine terephthalaldehyde }
\end{array} \\
& \text { 1-substituted- } \\
& \text { 4-ethynylbenzene } \\
& \overbrace{\mathrm{N}}^{\mathrm{NH}_{2}} \\
& \text { 4,5-disubstituted ||| isophthalaldehyde }
\end{aligned}
$$
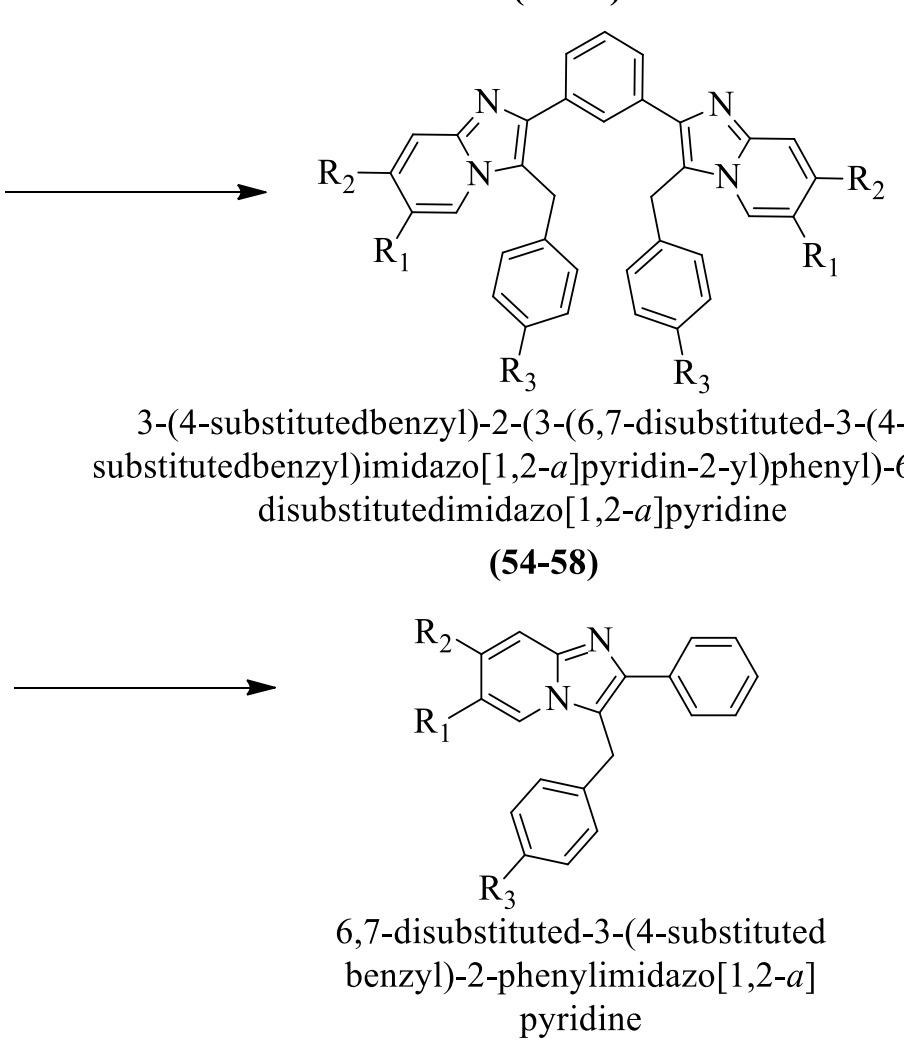

Reagents and conditions: (i) EtOH, $10 \mathrm{~min}, \mathrm{CuSO}_{4} .5 \mathrm{H}_{2} \mathrm{O}$, D-glucose, reflux, $10 \mathrm{hr}$ 


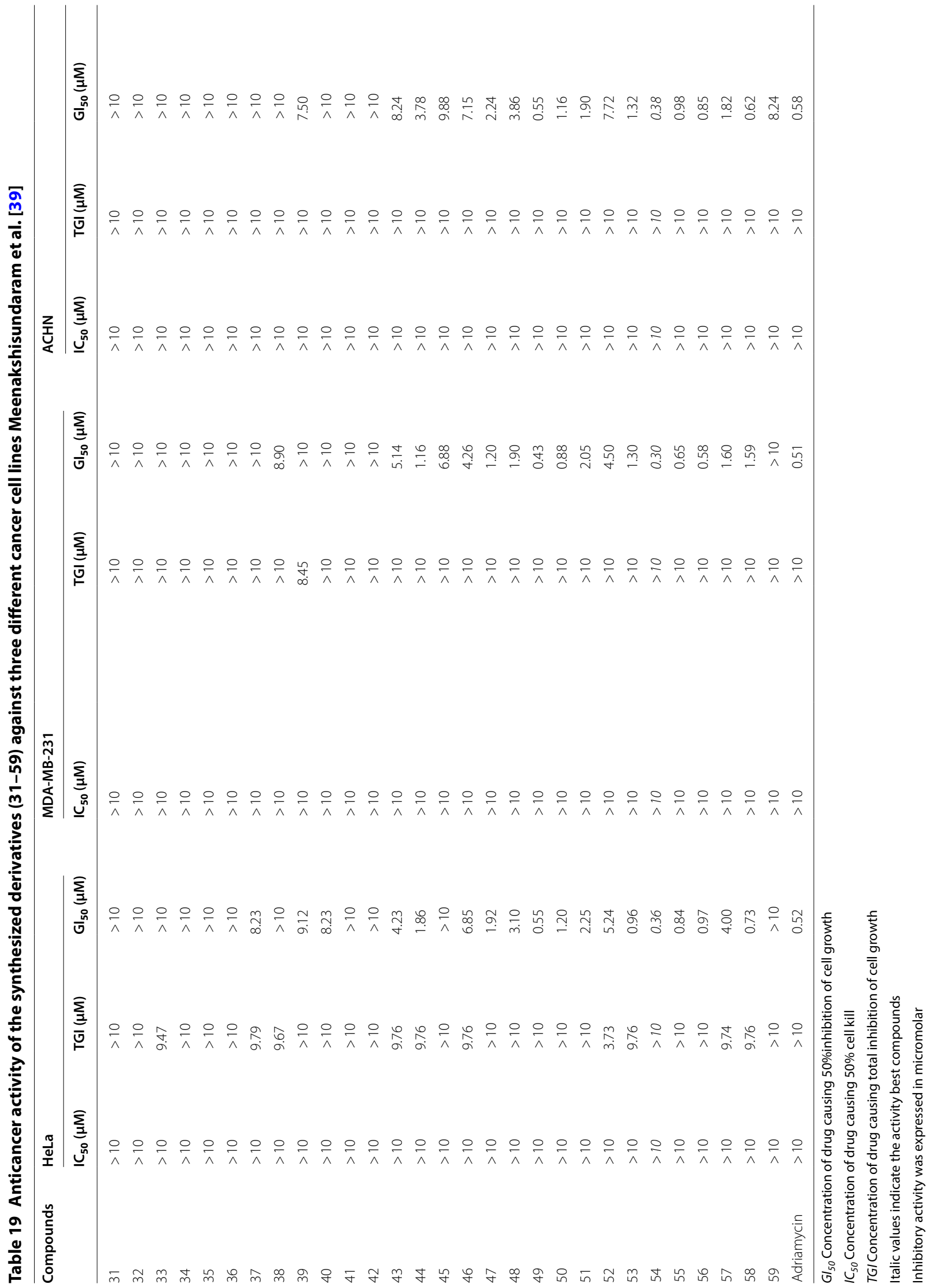




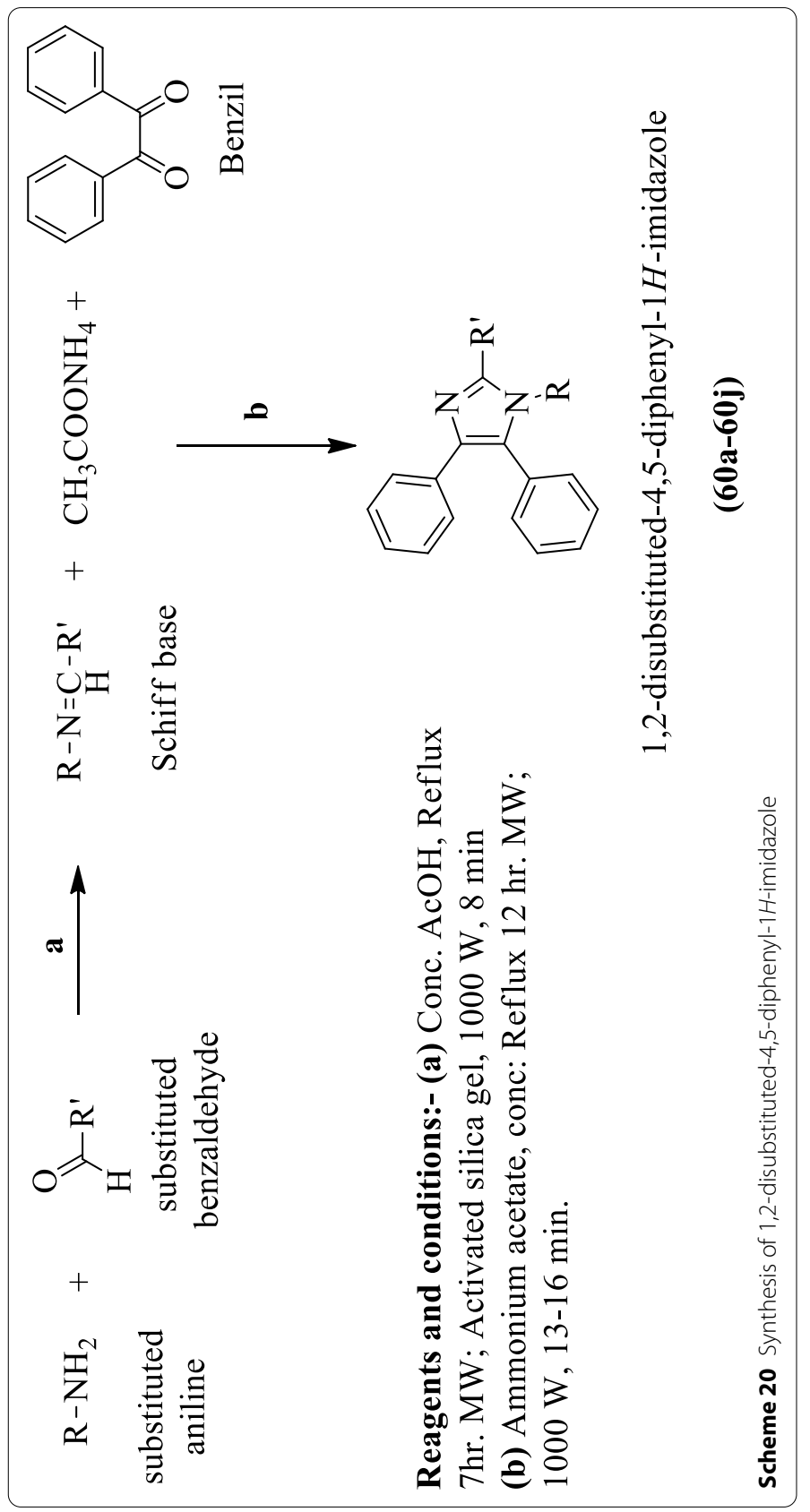


Table 20 Antitumor activity of the synthesized derivatives (60a-j) Sharma et al. [40]

\begin{tabular}{|c|c|c|c|c|}
\hline Compounds & Substituent $\mathrm{R}$ & Substituent $\mathrm{R}^{\prime}$ & DLA cells $\mathrm{CTC}_{50} \mu \mathrm{g} / \mathrm{mL}$ & $\begin{array}{l}\text { EAC cells } \\
\mathrm{CTC}_{50} \mu \mathrm{g} / \\
\mathrm{mL}\end{array}$ \\
\hline $60 a$ & $-\mathrm{NH}$ & & 190.26 & 60.50 \\
\hline $60 \mathrm{~b}$ & $-\mathrm{NH}$ & & 114.00 & 240.00 \\
\hline $60 c$ & $-\mathrm{NH}$ & $\mathrm{CH}_{3}$ & 98.56 & 31.25 \\
\hline $60 d$ & $-\mathrm{NH}$ & $\mathrm{Cl}$ & 309.67 & 200.22 \\
\hline $60 e$ & & & $>500$ & 489.34 \\
\hline $60 f$ & & $\mathrm{Cl}$ & 207.60 & 115.31 \\
\hline 60 & & & 238.50 & 31.25 \\
\hline $60 \mathrm{~h}$ & & & $>500$ & $>500$ \\
\hline $60 i$ & & $\mathrm{Cl}$ & 405.68 & 305.91 \\
\hline $60 j$ & & & 150.26 & 94.63 \\
\hline
\end{tabular}




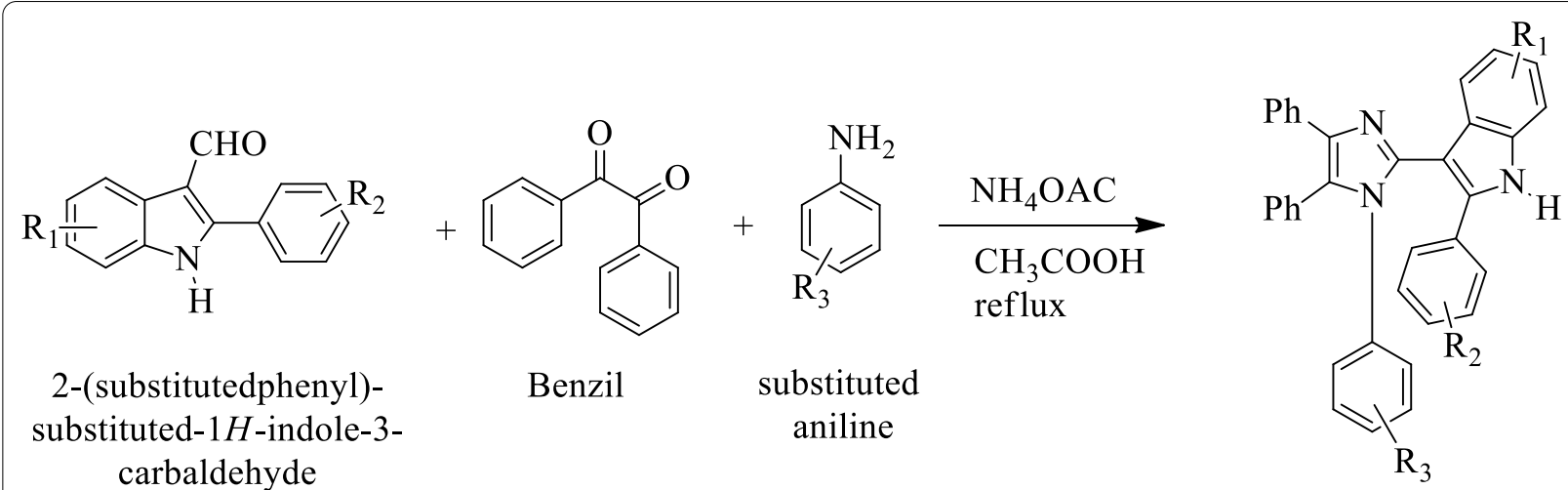

3-(4,5-diphenyl-1-(substitute phenyl)

-1H-imidazol-2-yl)-substituted-2-(substituted phenyl)- $1 H$-indole

(61a-j)

Scheme 21 Synthesis of 3-(4,5-diphenyl-1-(substitute phenyl)-1H-imidazol-2-yl)-sunstituted-2-(substitutedphenyl)- $1 H$-indole

Table 21 Antioxidant activity of the synthesized derivatives (61aj) Naureen et al. [41]

\begin{tabular}{|c|c|c|c|c|c|}
\hline \multirow[t]{2}{*}{ Compounds } & \multirow[t]{2}{*}{$\mathrm{R}_{1}$} & \multirow[t]{2}{*}{$\mathbf{R}_{2}$} & \multirow[t]{2}{*}{$\mathbf{R}_{3}$} & \multicolumn{2}{|l|}{ Antioxidant activity } \\
\hline & & & & Inhibition (\%) at $0.5 \mathrm{mM}$ & $\mathrm{IC}_{50}(\mu \mathrm{M})$ \\
\hline $61 \mathrm{a}$ & $\mathrm{H}$ & $\mathrm{Cl}$ & $\mathrm{CH}_{3}$ & $62.58 \pm 0.7$ & $175.26 \pm 1.24$ \\
\hline $61 b$ & $\mathrm{H}$ & $\mathrm{Cl}$ & $\mathrm{Br}$ & $71.74 \pm 0.2$ & $146.27 \pm 1.09$ \\
\hline $61 c$ & $\mathrm{Br}$ & $H$ & $\mathrm{~F}$ & $71.87 \pm 0.5$ & $181.26 \pm 1.1$ \\
\hline $61 d$ & $\mathrm{H}$ & $\mathrm{Br}$ & $\mathrm{CH}_{3}$ & $90.39 \pm 0.5$ & $148.26 \pm 1.2$ \\
\hline $61 e$ & $\mathrm{H}$ & $\mathrm{Br}$ & $\mathrm{Cl}$ & $20.97 \pm 0.5$ & - \\
\hline $61 f$ & $\mathrm{H}$ & $\mathrm{CH}_{3}$ & $H$ & $67.61 \pm 0.3$ & $162.27 \pm 1.2$ \\
\hline $61 \mathrm{~g}$ & $\mathrm{H}$ & $\mathrm{CH}_{3}$ & $\mathrm{CH}_{3}$ & $44.21 \pm 0.7$ & - \\
\hline $61 \mathrm{~h}$ & $\mathrm{H}$ & $\mathrm{CH}_{3}$ & $\mathrm{Br}$ & $7.11 \pm 0.2$ & - \\
\hline $61 i$ & $\mathrm{H}$ & $\mathrm{CH}_{3}$ & $\mathrm{~F}$ & $18.91 \pm 0.6$ & - \\
\hline $61 j$ & $\mathrm{H}$ & $\mathrm{CH}_{3}$ & $\mathrm{OCH}_{3}$ & $23.03 \pm 0.5$ & - \\
\hline Thiourea & & & & - & - \\
\hline Quercetin & & & & $93.21 \pm 0.9$ & $16.96 \pm 0.1$ \\
\hline
\end{tabular}


a

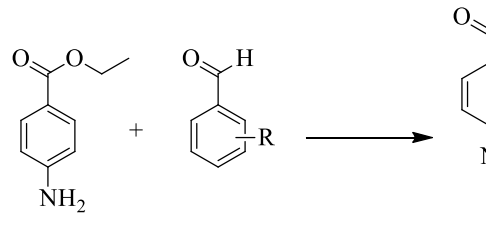

ethyl 4-

substituted aminobenzoate benzaldehyde<smiles>CCOC(=O)c1ccc(/N=C/c2ccccc2)cc1</smiles>

(E)-ethyl 4-((substituted benzylidene)amino)

benzoate

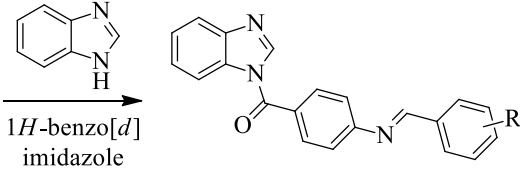

(E)-(1H-benzo[ $d]$ imidazol-1-yl)(4((substitutedbenzylidene)amino) phenyl)methanone

$(62 a, 62 b)$

b

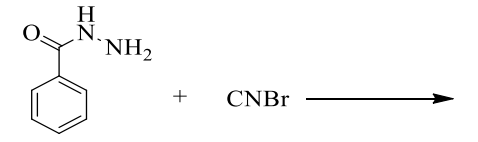

benzohydrazide

cyanic bromide

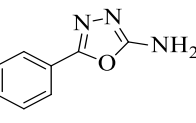

5-phenyl-1,3,4oxadiazol-2-amine

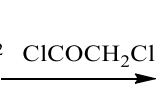<smiles>O=C(CCl)Nc1nnc(-c2ccccc2)o1</smiles>

2-chloro- $N$-(5-phenyl-1,3,4oxadiazol-2-yl)acetamide<smiles>c1cnc2nc[nH]c2c1</smiles><smiles></smiles><smiles></smiles>

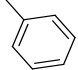

2-(1H-benzo[d]imidazol-1-yl)- $N-(5-$ phenyl-1,3,4-oxadiazol-2-yl)acetamide

(62c)

\section{c}

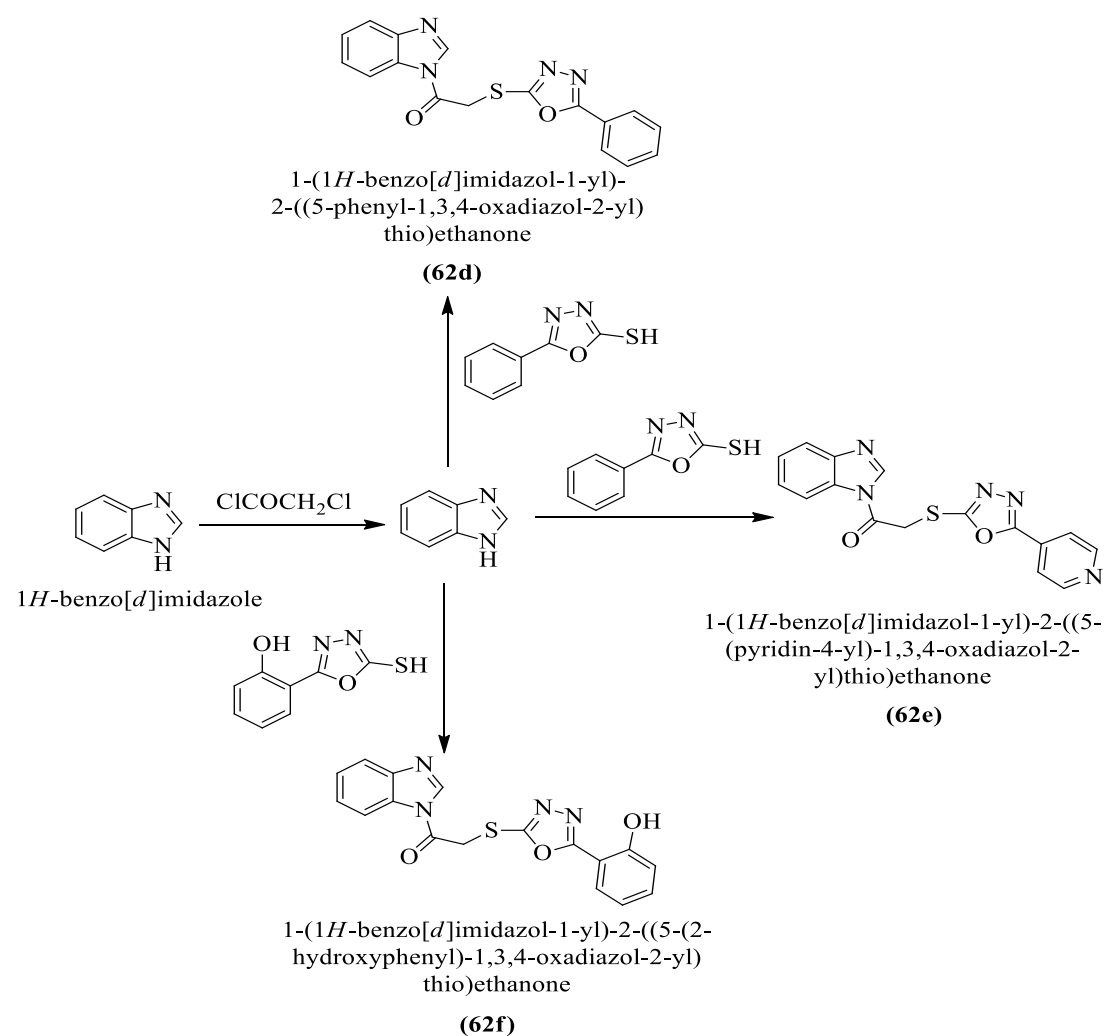

(62f)

Scheme 22 a Synthesis of $(E)-(1 H$-benzol[d]imidazole-1-yl)(4-(substitutedbenzylidene)amino)phenyl)methanone. b Synthesis of 2-(1H-benzol[ $d]$ imi dazole-1-yl)-N-(5-phenyl-1,3,4-oxadiazol-2-yl)acetamide. c Synthesis of substituted imidazole linked 1,3,4-oxadiazole derivatives 


\begin{tabular}{|c|c|c|c|c|}
\hline \multirow[t]{2}{*}{ Compounds } & \multicolumn{4}{|c|}{$\%$ Inhibition } \\
\hline & $10 \mu \mathrm{g} / \mathrm{ml}$ & $20 \mu \mathrm{g} / \mathrm{ml}$ & $30 \mu \mathrm{g} / \mathrm{ml}$ & $40 \mu \mathrm{g} / \mathrm{ml}$ \\
\hline $62 a$ & 7.20 & 12.30 & 37.65 & 39.42 \\
\hline $62 b$ & 34.77 & 34.66 & 37.65 & 39.42 \\
\hline $62 c$ & 7.08 & 15.61 & 21.04 & 22.26 \\
\hline $62 d$ & 17.71 & 29.34 & 30.34 & 40.86 \\
\hline $62 e$ & 34.77 & 37.76 & 47.17 & 52.16 \\
\hline $62 f$ & 18.98 & 24.67 & 28.90 & 34.34 \\
\hline Ascorbic acid & 56.03 & 58.80 & 65.33 & 68.55 \\
\hline
\end{tabular}<smiles>O=c1oc(-c2ccccc2)nc2ccccc12</smiles>

2-phenyl-4H-benzo

$[d][1,3]$ oxazin-4-one<smiles></smiles>

3-(2-aminoethyl)-2phenylquinazolin-4(3H)-one

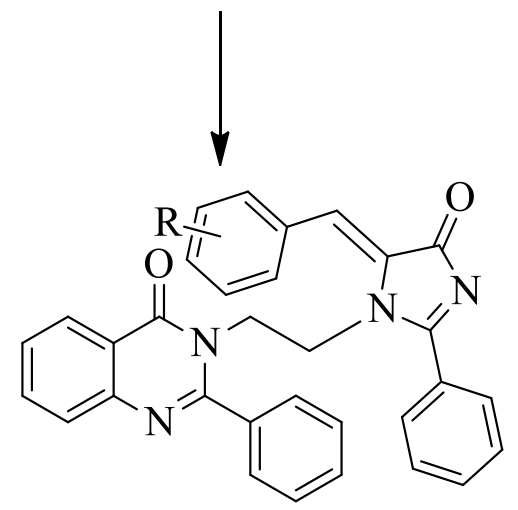<smiles>Nc1nc2ccccc2c(=O)n1-c1ccccc1</smiles>

3-amino-2-phenyl quinazolin-4(3H)-one<smiles>[R][X]C(C)CCSc1nnc(-c2ccccc2)n1-n1c(-c2ccccc2)nc2ccccc2c1=O</smiles>

3-(3-mercapto-5-(substituted phenyl) -4H-1,2,4-triazol-4-yl)-2-phenylquinazolin $-4(3 H)$-one

(63f-h)

(Z)-3-(2-(5-(benzylidene)-4-oxo-2phenyl-4,5-dihydro- $1 H$-imidazol-1-yl)ethyl)

-2-phenylquinazolin-4(3H)-one

\section{(63a-e)}

Scheme 23 Synthesis of (Z)-3-(2-(5-(3-methyl benzylidene)-4-oxo-2-phenyl-4, 5-dihydro-1H-imidazol-1-yl) ethyl)-2-phenyl quinazolin-4(3H)-one and 3-(3-mercapto-5-(susbstituted phenyl)-4H-1,2,4-triazol-4-yl)-2-phenylquinazolin-4(3H)-one 


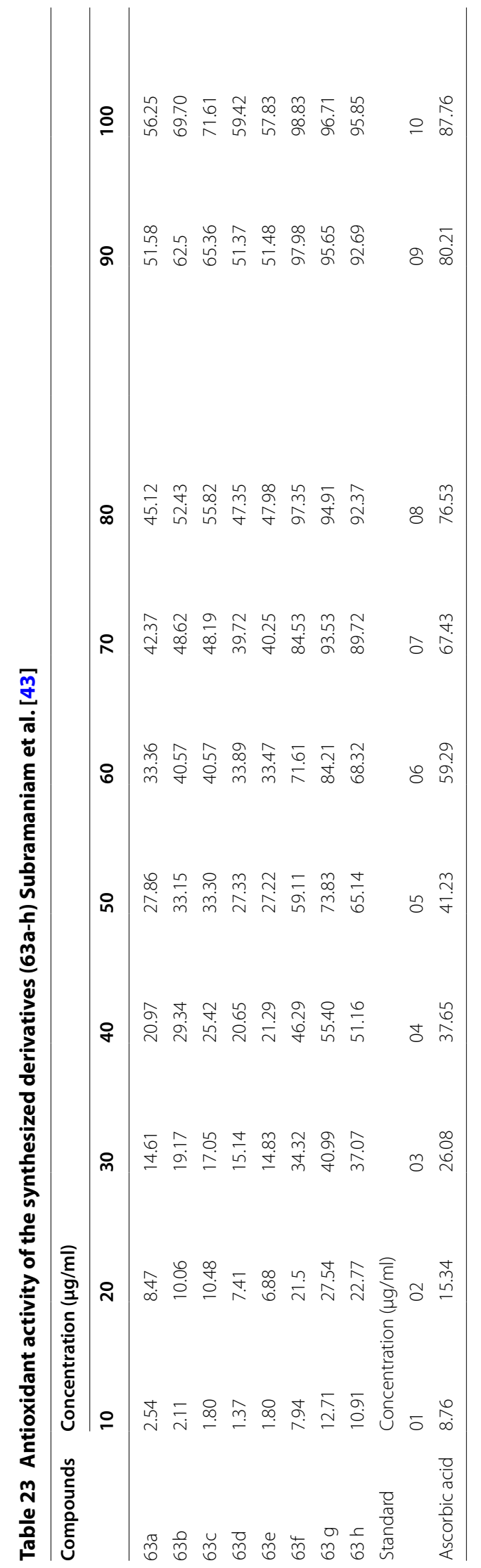




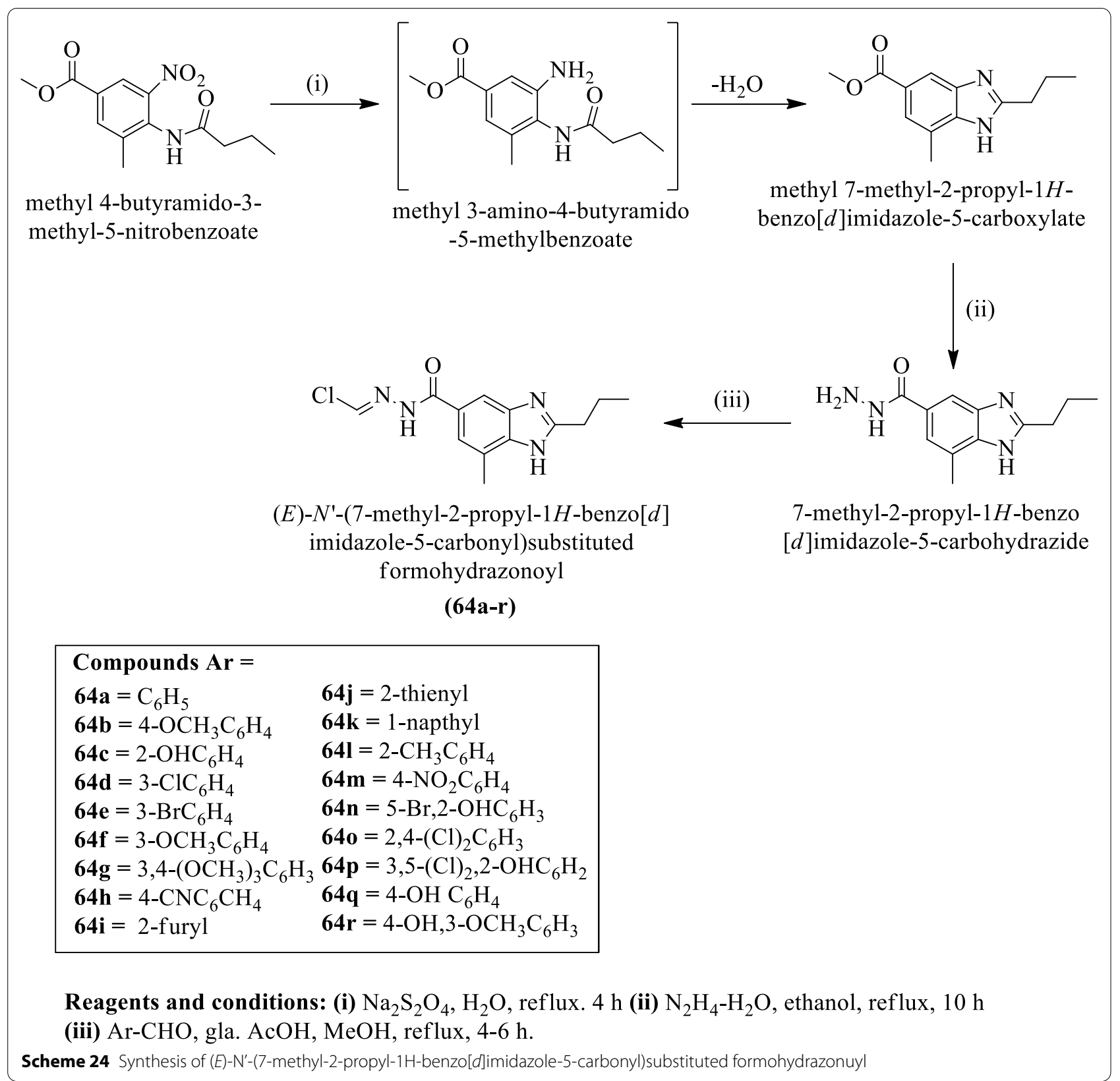


Table 24 Antioxidant activity of synthesized derivatives (64a-r) Katikireddy et al. [21]

\begin{tabular}{lr}
\hline Compounds & $\boldsymbol{I C}_{\mathbf{5 0}}(\boldsymbol{\mu \mathbf { g }} / \mathbf{m l})$ \\
\hline $64 \mathrm{a}$ & $49.28 \pm 3.03$ \\
$64 \mathrm{~b}$ & $32.17 \pm 2.87$ \\
$64 \mathrm{c}$ & $29.10 \pm 1.60$ \\
$64 \mathrm{~d}$ & $18.31 \pm 1.38$ \\
$64 \mathrm{e}$ & $26.81 \pm 2.10$ \\
$64 \mathrm{f}$ & $29.96 \pm 2.81$ \\
$64 \mathrm{~g}$ & $24.79 \pm 3.03$ \\
$64 \mathrm{~h}$ & $30.83 \pm 2.93$ \\
$64 \mathrm{i}$ & $23.19 \pm 1.72$ \\
$64 \mathrm{j}$ & $30.08 \pm 2.60$ \\
$64 \mathrm{k}$ & $20.05 \pm 1.27$ \\
$64 \mathrm{l}$ & $25.97 \pm 2.18$ \\
$64 \mathrm{~m}$ & $13.60 \pm 1.37$ \\
$64 \mathrm{n}$ & $9.40 \pm 1.04$ \\
640 & $12.39 \pm 1.26$ \\
$64 \mathrm{p}$ & $16.27 \pm 1.39$ \\
$64 \mathrm{q}$ & $24.70 \pm 2.29$ \\
$64 \mathrm{r}$ & $38.28 \pm 3.07$ \\
Ascorbic acid & $7.50 \pm 0.89$ \\
\hline
\end{tabular}

Patel et al. [53] synthesized 6-(substituted phenyl)-2-(1methyl-1H-imidazol-2-yl) imidazo [2,1-b] [1,3,4] thiadiazole (Scheme 36) and evaluated for anti-tubercular activity against Mycobacterium tuberculosis and MIC values of these derivatives were calculated. The conclusion of anti-tubercular activity was presented in (Table 36, Patel et al. [53]).

Yadav et al. [54] synthesized 2-((1-benzoyl-1H-benzo[d] imidazol-2-yl) thio)-N-(substituted phenyl) acetamide (Scheme 37) and evaluated for anti-tubercular activity against Mycobacterium tuberculosis strain and MIC values of these derivatives were calculated. Streptomycin was used as a reference drug and the results of anti-tubercular activity were presented in (Table 37, Yadav et al. [54]).

\section{Conclusion}

In this present review article, we have summarized different pharmacological activities of 1,3-diazole containing compounds. From this study, we have found that 1,3-diazole containing compounds can be synthesized by various kinds of synthetic routes, and these derivatives having a wide range of biological activities such as antitumor, antitubercular, antimicrobial, antihypertensive and 
$\mathbf{a}$

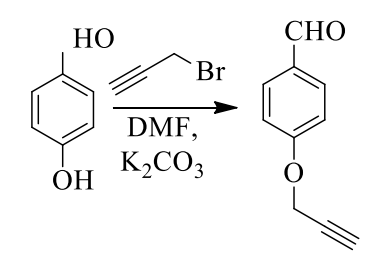

4-hydroxy benzaldehyde 4-(prop-2-yn-1yloxy)benzaldehyde<smiles>[R]c1ccc(N)c([R])c1[R1]</smiles>
MW

4-((1-(2,3,4-trisubstituted phenyl)-1 $H$-1,2,3-triazol-4-yl) methoxy)benzaldehyde

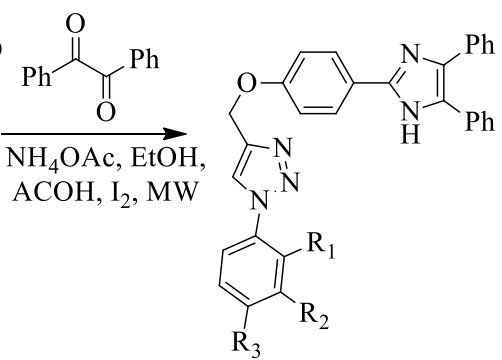

4-((4-(4,5-diphenyl-1 $H$-imidazol2-yl)phenoxy)methyl)-1-(2,3,4trisubstitutedphenyl)- $1 \mathrm{H}$ 1,2,3-triazole

(65a-h)

65a: $\mathrm{R}_{1}=\mathrm{H}, \mathrm{R}_{2}=\mathrm{Cl}, \mathrm{R}_{3}=\mathrm{H}$

65b: $\mathrm{R}_{1}=\mathrm{H}, \mathrm{R}_{2}=\mathrm{H}, \mathrm{R}_{3}=\mathrm{Cl}$

65c: $R_{1}=H, R_{2}=H, R_{3}=M e$

65e: $\mathrm{R}_{1}=\mathrm{OMe}, \mathrm{R}_{2}=\mathrm{H}, \mathrm{R}_{3}=\mathrm{NO}_{2}$

65d: $\mathrm{R}_{1}=\mathrm{OMe}, \mathrm{R}_{2}=\mathrm{H}, \mathrm{R}_{3}=\mathrm{H}$

65f: $\mathrm{R}_{1}=\mathrm{NO}_{2}, \mathrm{R}_{2}=\mathrm{H}, \mathrm{R}_{3}=\mathrm{OMe}$

65g: $R_{1}=N_{2}, R_{2}=H, R_{3}=H$

65h: $\mathrm{R}_{1}=\mathrm{H}, \mathrm{R}_{2}=\mathrm{H}, \mathrm{R}_{3}=\mathrm{NO}_{2}$

b<smiles>O=Cc1ccc(O)cc1</smiles>

4-hydroxy benzaldehyde<smiles>[R]c1ccc(-n2cc(COc3ccc(-c4nc(-c5ccccc5)c(-c5ccccc5)n4Cc4cn(-c5ccc([R3])c([R2])c5[R])nn4)cc3)nn2)c([R])c1[R]</smiles>

4-((4-(4,5-diphenyl-1-((1-(2,3,4-trisubstitutedphenyl) -1H-1,2,3-triazol-4-yl)methyl)- $1 H$-imidazol-2-yl) phenoxy)methyl)-1-(2,3,4-trisubstitutedphenyl)-

$1 H$-1,2,3-triazole<smiles>CC(C)(C(N)=O)C(=O)NN</smiles>

4-(4,5-diphenyl-1 $H$-imidazol2-yl)phenol

\section{(66a-h)}

$$
\begin{aligned}
& \text { 66a: } \mathrm{R}_{1}=H, \mathrm{R}_{2}=\mathrm{Cl}, \mathrm{R}_{3}=\mathrm{H} \\
& \text { 66b: } \mathrm{R}_{1}=H, \mathrm{R}_{2}=H, \mathrm{R}_{3}=\mathrm{Cl} \\
& \text { 66c: } \mathrm{R}_{1}=H, \mathrm{R}_{2}=H, \mathrm{R}_{3}=\mathrm{Me} \\
& \text { 66d: } \mathrm{R}_{1}=\mathrm{OMe}, \mathrm{R}_{2}=\mathrm{H}, \mathrm{R}_{3}=\mathrm{H} \\
& \text { 66e: } \mathrm{R}_{1}=\mathrm{OMe}, \mathrm{R}_{2}=\mathrm{H}, \mathrm{R}_{3}=\mathrm{OMe} \\
& \text { 66f: } \mathrm{R}_{1}=\mathrm{NO}_{2}, \mathrm{R}_{2}=\mathrm{H}, \mathrm{R}_{3}=\mathrm{OMe} \\
& \text { 66g: } \mathrm{R}_{1}=\mathrm{NO}_{2}, \mathrm{R}_{2}=H, \mathrm{R}_{3}=\mathrm{NO}_{2} \\
& \text { 66h: } \mathrm{R}_{1}=H, \mathrm{R}_{2}=\mathrm{H}, \mathrm{R}_{3}=\mathrm{NO}_{2}
\end{aligned}
$$

Scheme 25 a Synthesis of 4-((4-(4,5-diphenyl-1H-imidazol-2-yl)phenoxy)methyl)-1-(2, 3, 4-trisubstituted phenyl)-1H-1, 2, 3-triazole; b Synthesis of 4-((4-(4,5-diphenyl-1-((1-(2, 3, 4-trisubstituted phenyl)-1H-1, 2, 3-triazole-4-yl)methyl)-1H-imiidazol-2-yl))phenoxy)methyl)-1-(2,3,4-trisubstitutedphe nyl)-1H-1,2,3-triazole 
Table 25 (a) DPPH radical scavenging activity of (65a-h) and (66a-h); (b) Hydrogen peroxide radical scavenging activity of (65a-h) and (66a-h); (c) Nitric oxide radical scavenging activity of (65a-h) and (66a-h); (d) FRAP oxide radical scavenging activity of (65a-h) and (66a-h) Subhashini et al. [44]

\begin{tabular}{llll}
\hline Compounds & \multicolumn{3}{l}{ Concentration } \\
\cline { 2 - 3 } & $10 \mu \mathrm{ml}$ & $50 \mu \mathrm{g} / \mathrm{ml}$ & $100 \mu \mathrm{g} / \mathrm{ml}$
\end{tabular}

(a)

\begin{tabular}{|c|c|c|c|c|}
\hline $65 a$ & 57 & 71 & 81 & 94 \\
\hline $65 b$ & 49 & 55 & 59 & 63 \\
\hline $65 c$ & 42 & 53 & 65 & 69 \\
\hline $65 d$ & 53 & 59 & 64 & 93 \\
\hline $65 e$ & 35 & 42 & 55 & 63 \\
\hline $65 f$ & 44 & 61 & 79 & 90 \\
\hline $65 \mathrm{~g}$ & 41 & 49 & 53 & 61 \\
\hline $65 \mathrm{~h}$ & 67 & 75 & 83 & 91 \\
\hline $66 a$ & 55 & 63 & 71 & 87 \\
\hline $66 b$ & 60 & 69 & 76 & 89 \\
\hline $66 c$ & 69 & 78 & 81 & 95 \\
\hline $66 d$ & 48 & 67 & 79 & 85 \\
\hline $66 e$ & 71 & 79 & 85 & 96 \\
\hline $66 f$ & 33 & 44 & 55 & 61 \\
\hline $66 \mathrm{~g}$ & 41 & 47 & 59 & 62 \\
\hline $66 \mathrm{~h}$ & 66 & 74 & 81 & 90 \\
\hline Standard & 85 & 89 & 93 & 97 \\
\hline
\end{tabular}

(b)

\begin{tabular}{|c|c|c|c|c|}
\hline $65 a$ & 49 & 67 & 75 & 87 \\
\hline $65 b$ & 59 & 73 & 81 & 92 \\
\hline $65 c$ & 40 & 49 & 55 & 57 \\
\hline $65 d$ & 47 & 65 & 72 & 89 \\
\hline $65 e$ & 31 & 43 & 49 & 56 \\
\hline $65 f$ & 52 & 73 & 81 & 92 \\
\hline $65 \mathrm{~g}$ & 35 & 43 & 51 & 63 \\
\hline $65 \mathrm{~h}$ & 57 & 68 & 75 & 88 \\
\hline $66 a$ & 51 & 63 & 78 & 91 \\
\hline $66 \mathrm{~b}$ & 54 & 71 & 82 & 90 \\
\hline $66 c$ & 71 & 88 & 91 & 96 \\
\hline $66 d$ & 57 & 73 & 85 & 94 \\
\hline $66 \mathrm{e}$ & 37 & 45 & 52 & 59 \\
\hline $66 f$ & 65 & 78 & 86 & 94 \\
\hline $66 \mathrm{~g}$ & 38 & 45 & 53 & 55 \\
\hline $66 \mathrm{~h}$ & 57 & 65 & 78 & 86 \\
\hline Standard & 83 & 91 & 95 & 98 \\
\hline
\end{tabular}

(c)

\begin{tabular}{lllll}
\hline $65 \mathrm{a}$ & 49 & 55 & 63 & 78 \\
$65 \mathrm{~b}$ & 54 & 69 & 75 & 89 \\
$65 \mathrm{c}$ & 31 & 37 & 44 & 51 \\
$65 \mathrm{~d}$ & 56 & 68 & 79 & 85 \\
$65 \mathrm{e}$ & 29 & 36 & 41 & 47 \\
$65 f$ & 48 & 56 & 67 & 74 \\
$65 \mathrm{~g}$ & 23 & 32 & 39 & 43 \\
$65 \mathrm{~h}$ & 61 & 77 & 86 & 95 \\
\hline
\end{tabular}


Table 25 (continued)

\begin{tabular}{|c|c|c|c|c|}
\hline \multicolumn{5}{|l|}{ (c) } \\
\hline $66 a$ & 65 & 75 & 82 & 89 \\
\hline $66 b$ & 57 & 69 & 79 & 87 \\
\hline $66 c$ & 68 & 79 & 88 & 91 \\
\hline $66 \mathrm{~d}$ & 57 & 68 & 75 & 88 \\
\hline $66 \mathrm{e}$ & 25 & 37 & 42 & 46 \\
\hline $66 f$ & 48 & 55 & 67 & 78 \\
\hline $66 \mathrm{~g}$ & 21 & 27 & 33 & 39 \\
\hline $66 \mathrm{~h}$ & 67 & 65 & 77 & 86 \\
\hline Standard & 81 & 86 & 91 & 96 \\
\hline \multicolumn{5}{|l|}{ (d) } \\
\hline $65 a$ & 47 & 64 & 78 & 87 \\
\hline $65 b$ & 51 & 67 & 79 & 93 \\
\hline $65 c$ & 31 & 39 & 43 & 47 \\
\hline $65 d$ & 63 & 77 & 83 & 92 \\
\hline $65 \mathrm{e}$ & 22 & 27 & 32 & 38 \\
\hline $65 f$ & 57 & 68 & 77 & 85 \\
\hline $65 \mathrm{~g}$ & 27 & 33 & 40 & 45 \\
\hline $65 \mathrm{~h}$ & 49 & 58 & 69 & 87 \\
\hline $66 a$ & 56 & 63 & 75 & 89 \\
\hline $66 b$ & 49 & 58 & 67 & 85 \\
\hline $66 c$ & 65 & 71 & 84 & 97 \\
\hline $66 d$ & 64 & 79 & 86 & 91 \\
\hline $66 \mathrm{e}$ & 30 & 37 & 45 & 50 \\
\hline $66 f$ & 45 & 53 & 62 & 85 \\
\hline $66 \mathrm{~g}$ & 31 & 39 & 42 & 48 \\
\hline $66 \mathrm{~h}$ & 60 & 69 & 78 & 87 \\
\hline Standard & 88 & 92 & 95 & 99 \\
\hline
\end{tabular}




4-(trifluoromethyl)
benzene-1,2-diamine

Table 26 Antihypertensive activity of the synthesized derivatives (67a-o) in SHR Navarrete-Vázquez et al. [45]

\begin{tabular}{|c|c|c|c|c|c|c|c|c|}
\hline \multirow[t]{3}{*}{ Compounds } & \multirow[t]{3}{*}{ R1 } & \multirow[t]{3}{*}{ R2 } & \multirow[t]{3}{*}{ R3 } & \multirow[t]{3}{*}{ R4 } & \multicolumn{4}{|c|}{ Ex vivo vasorelaxant effect } \\
\hline & & & & & \multicolumn{2}{|c|}{ With endothelium (+E) } & \multicolumn{2}{|c|}{ Without endothelium (-E) } \\
\hline & & & & & $\mathrm{EC50}(\mu \mathrm{M})$ & $E_{\max }(\%)$ & $\mathrm{EC50}(\mu \mathrm{M})$ & $E_{\max }(\%)$ \\
\hline $67 a$ & $-\mathrm{CF}_{3}$ & $-\mathrm{H}$ & $-\mathrm{H}$ & $-\mathrm{H}$ & $369.37 \pm 10.2$ & $91.2 \pm 1.18$ & $467.75 \pm 73.6$ & $75.6 \pm 6.31$ \\
\hline $67 b$ & $-\mathrm{CF}_{3}$ & $-\mathrm{OMe}$ & $-\mathrm{H}$ & $-\mathrm{H}$ & $210.33 \pm 11.3$ & $75.14 \pm 33.5$ & $574.85 \pm 30.3$ & $45.7 \pm 15.4$ \\
\hline $67 c$ & $-\mathrm{CF}_{3}$ & -OEt & $-\mathrm{H}$ & $-\mathrm{H}$ & $548.5 \pm 27.8$ & $90.97 \pm 2.30$ & $548.51 \pm 77.1$ & $19.8 \pm 8.13$ \\
\hline $67 d$ & $-\mathrm{CF}_{3}$ & $-\mathrm{NO}_{2}$ & $-\mathrm{H}$ & $-\mathrm{H}$ & $3.18 \pm 0.30$ & $93.16 \pm 3.52$ & $15.03 \pm 7.59$ & $85.31 \pm 2.63$ \\
\hline $67 e$ & $-\mathrm{CF}_{3}$ & $-\mathrm{H}$ & $-\mathrm{H}$ & $-\mathrm{OH}$ & $219.20 \pm 14.1$ & $51.15 \pm 20.6$ & $219.20 \pm 71.6$ & $37.04 \pm 10.6$ \\
\hline $67 f$ & $-\mathrm{CF}_{3}$ & $-\mathrm{H}$ & $-\mathrm{H}$ & $-\mathrm{OPr}$ & $524.49 \pm 25.4$ & $51.0 \pm 7.33$ & $524.49 \pm 19.3$ & $19.0 \pm 6.01$ \\
\hline $67 g$ & $-\mathrm{CF}_{3}$ & $-H$ & $-H$ & $-\mathrm{N}(\mathrm{Me})_{2}$ & $550.27 \pm 30.1$ & $63.2 \pm 4.81$ & $550.27 \pm 84.5$ & $30.9 \pm 7.53$ \\
\hline $67 \mathrm{~h}$ & $-\mathrm{CF}_{3}$ & $-\mathrm{H}$ & -OMe & $-\mathrm{OH}$ & $34.84 \pm 5.43$ & $99.55 \pm 1.23$ & $140.14 \pm 63.2$ & $97.67 \pm 3.26$ \\
\hline $67 i$ & $-\mathrm{CF}_{3}$ & $-H$ & $-\mathrm{OCH}_{2} \mathrm{O}$ & - & $38.53 \pm 2.35$ & $101.17 \pm 5.83$ & $77.42 \pm 9.41$ & $99.6 \pm 13.5$ \\
\hline $67 j$ & $\mathrm{NO}_{2}$ & $-\mathrm{H}$ & $-\mathrm{H}$ & $-\mathrm{H}$ & $4.93 \pm 0.30$ & $73.82 \pm 5.37$ & $35.1 \pm 5.21$ & $60.53 \pm 5.58$ \\
\hline $67 k$ & $\mathrm{NO}_{2}$ & -OEt & $-\mathrm{H}$ & $-\mathrm{H}$ & $3.71 \pm 0.10$ & $84.82 \pm 3.73$ & $15.0 \pm 1.12$ & $46.35 \pm 7.85$ \\
\hline 671 & $\mathrm{NO}_{2}$ & $-\mathrm{OiPr}$ & $-\mathrm{H}$ & $-\mathrm{H}$ & $4.89 \pm 0.29$ & $80.71 \pm 9.41$ & $14.12 \pm 1.05$ & $31.69 \pm 1.32$ \\
\hline $67 m$ & $\mathrm{NO}_{2}$ & $-\mathrm{H}$ & -OMe & $-\mathrm{OH}$ & $1.81 \pm 0.08$ & $91.74 \pm 2.35$ & $19.49 \pm 1.79$ & $55.22 \pm 8.85$ \\
\hline $67 n$ & $\mathrm{NO}_{2}$ & $-\mathrm{H}$ & -OMe & $-\mathrm{OMe}$ & $2.5 \pm 0.10$ & $75.0 \pm 9.35$ & $301.9 \pm 10.2$ & $36.33 \pm 6.20$ \\
\hline 670 & $\mathrm{NO}_{2}$ & $-\mathrm{OMe}$ & -OMe & -OMe & $3.23 \pm 0.20$ & $90.0 \pm 4.56$ & $43.65 \pm 2.37$ & $58.91 \pm 7.81$ \\
\hline Pimobendan & & & & & $4.67 \pm 0.83$ & $93.22 \pm 5.23$ & N.T & N.T \\
\hline Carbachol & & & & & $0.51 \pm 1.9$ & $106.3 \pm 9.71$ & N.A & N.A \\
\hline Nitrendipine & & & & & N.T & N.T & $0.03 \pm 0.003$ & $98.90 \pm 5.0$ \\
\hline
\end{tabular}

N.T Not tested, N.A Not active 
<smiles>[R20]OC(=O)C1=C(C)NC(C)=C(C(=O)O[R20])C1c1cnc([R18])n1Cc1ccccc1</smiles>

4-(1-benzyl-2-(substitutedthio)-1H -imidazol-5-yl)-5-(substitutedcarbonyl) -2,6-dimethyl-1,4-dihydropyridine-

3-substitutedcarboxylic acid

$$
\begin{aligned}
& \mathbf{R}_{1}=\mathrm{CH}_{3}, \mathrm{C}_{2} \mathrm{H}_{5}, \mathrm{CH}_{2} \mathrm{C}_{6} \mathrm{H}_{5} \\
& \mathbf{R}_{2}=\mathrm{CH}_{3}, \mathrm{C}_{2} \mathrm{H}_{5},
\end{aligned}
$$<smiles>[R20]OC(=O)C1=C(C)NC(CCN(C)C)=C(C(=O)O[R20])C1c1cnc(S)n1Cc1ccccc1</smiles>

4-(1-benzyl-2-(substitutedthio)- $1 H$-imidazol-5-yl)2-(2-(dimethylamino)ethyl)-5-(substituted carbonyl)-6-methyl-1,4-dihydropyridine3-substitutedcarboxylic acid

(68a-f)<smiles>[R20]OC(=O)C1=C(C)NC(Cn2ccnc2)=C(C(=O)O[R20])C1c1cnc([Y])n1Cc1ccccc1</smiles>

2-(2-(1H-imidazol-1-yl)ethyl)-4-(1-benzyl-2(substitutedthio)-1H-imidazol-5-yl)-5-(substituted carbonyl)-6-methyl-1,4-dihydropyridine3-substitutedcarboxylic acid 


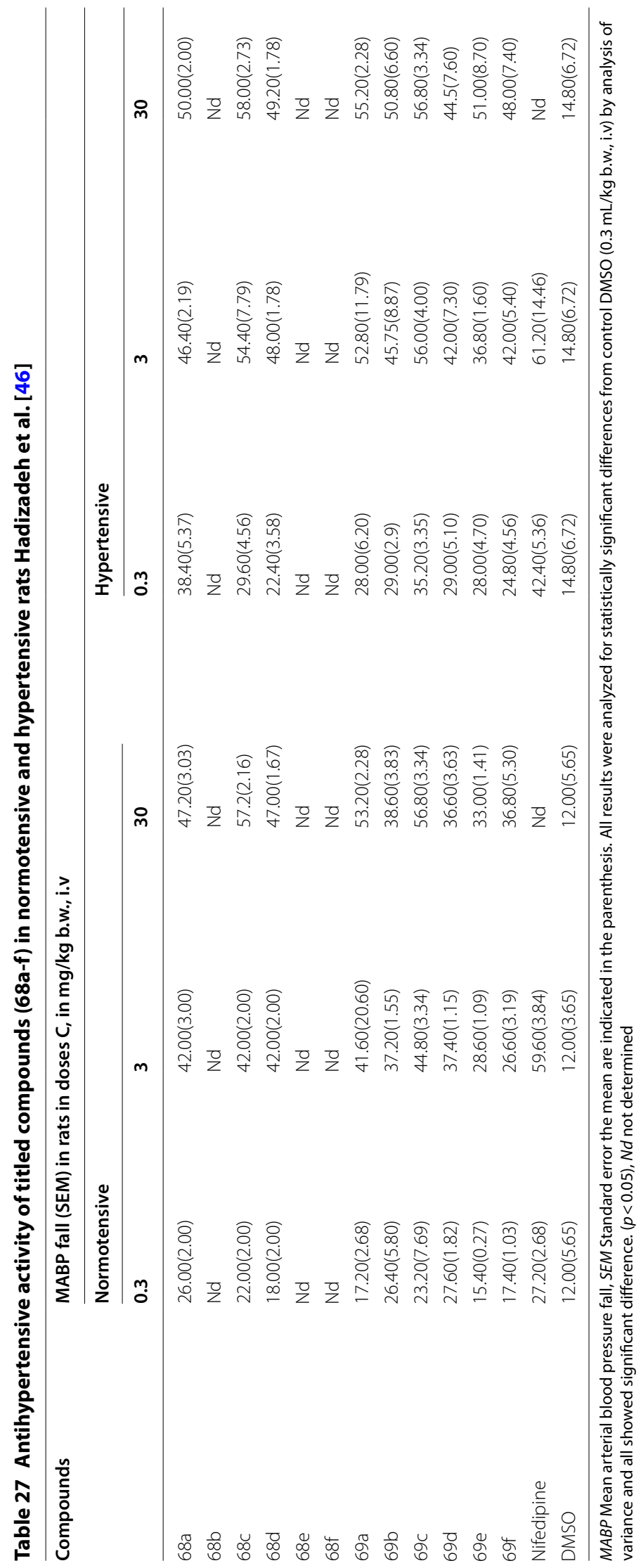




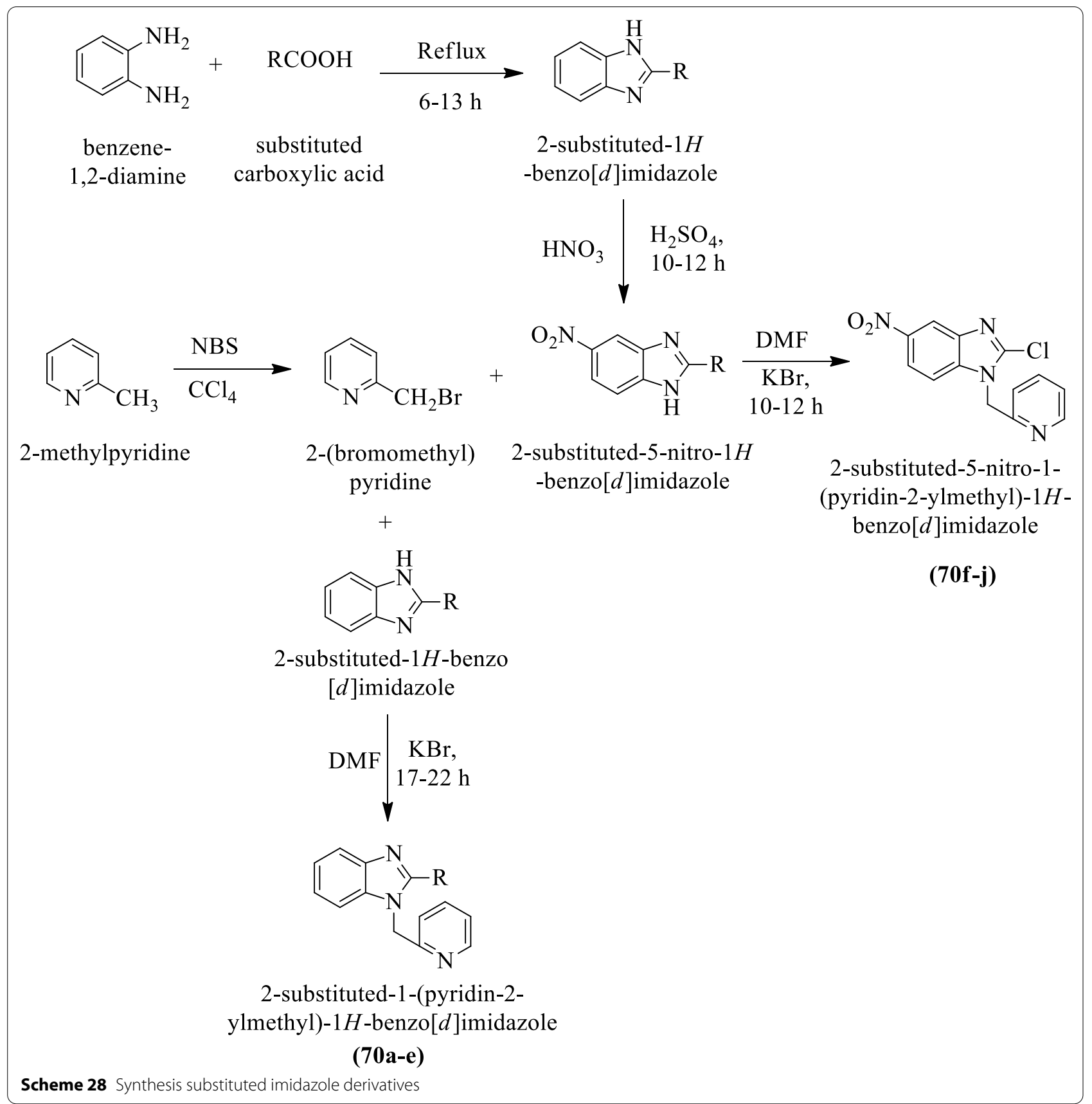




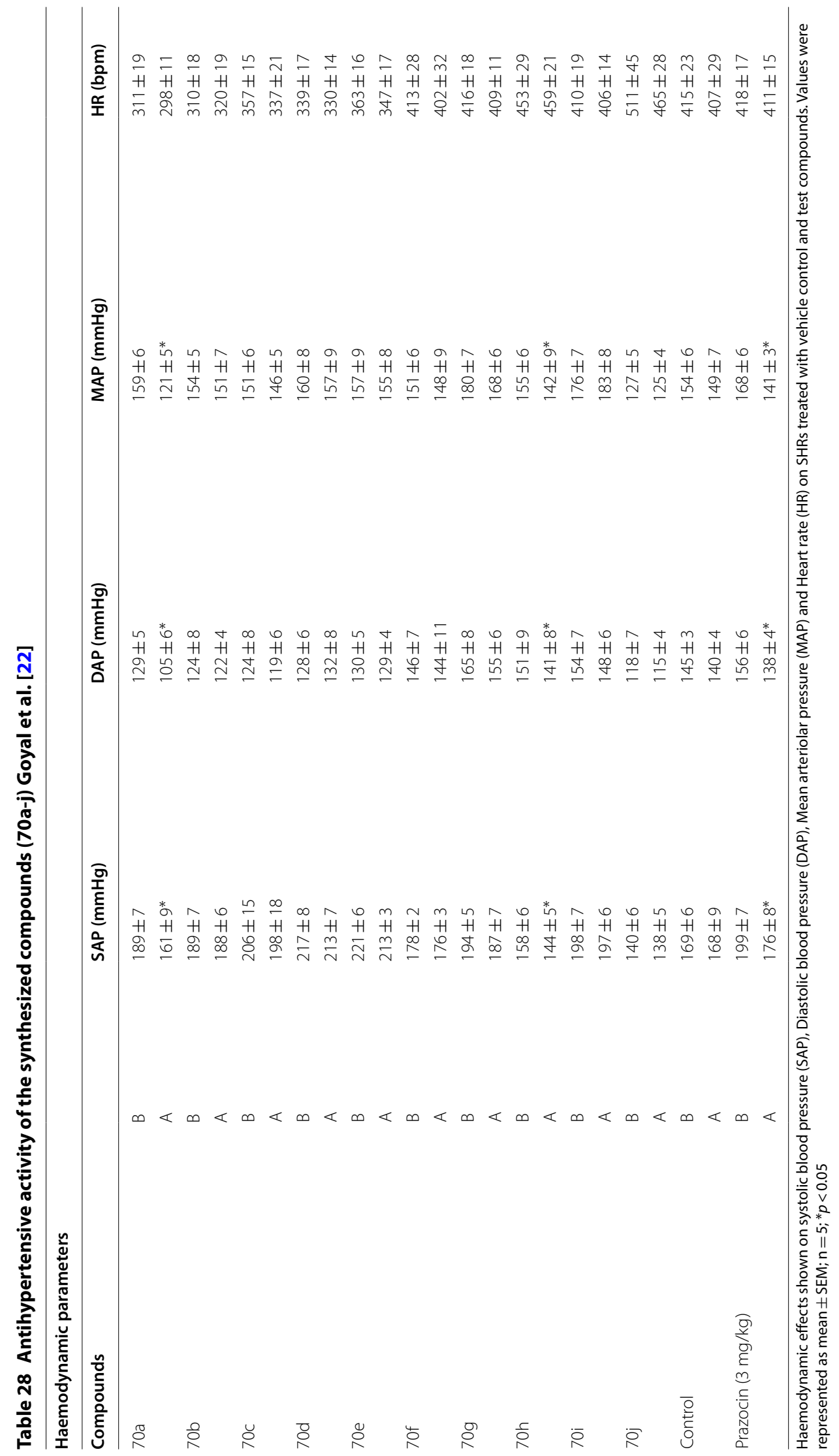




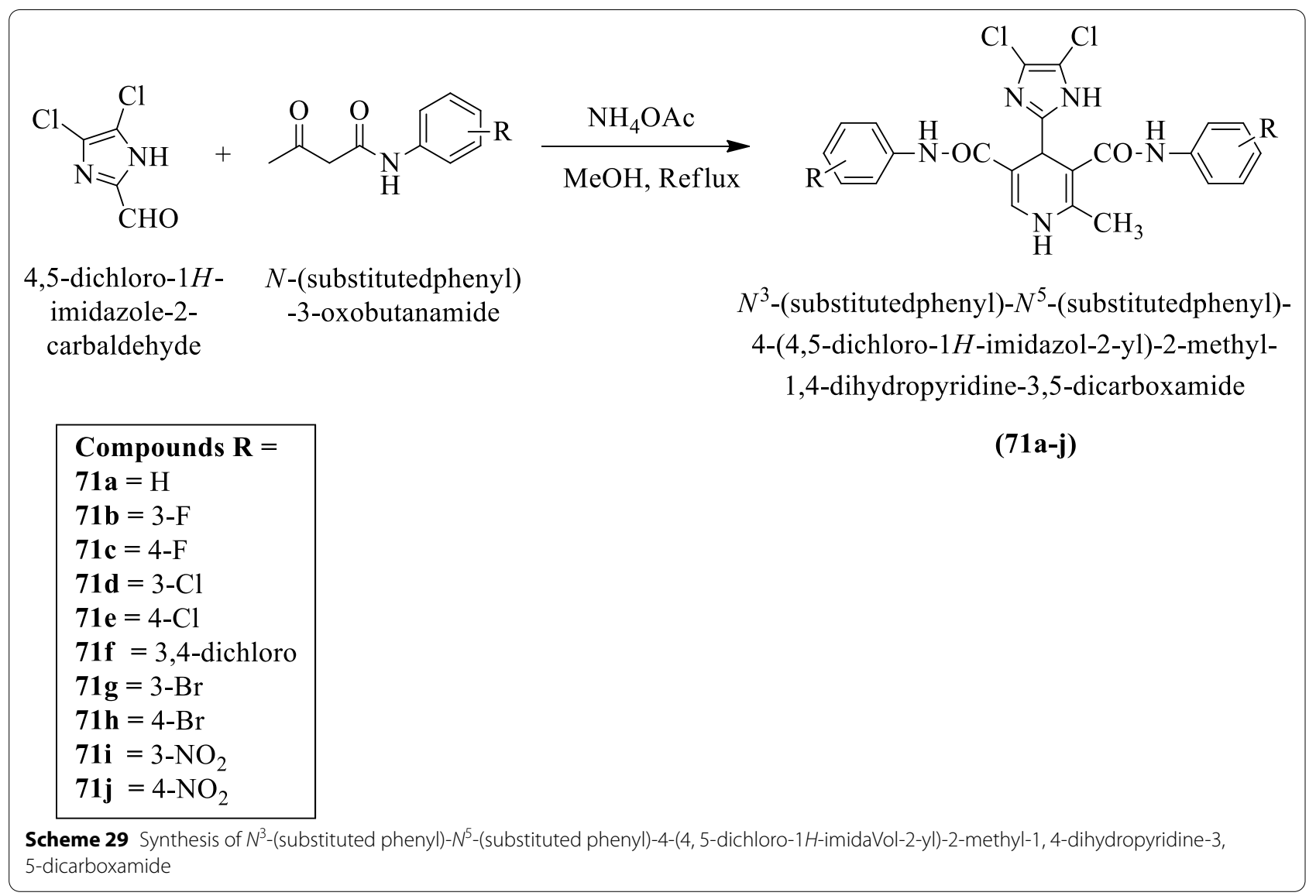

Table 29 Antitubercular activity of the synthesized compounds (71a-j) against Mycobacterium tuberculosis ( $\mathrm{H}_{37}$ Rv strain) Amini et al. [47]

\begin{tabular}{llc}
\hline Compounds & R & Inhibition \% \\
\hline $71 \mathrm{a}$ & $\mathrm{H}$ & 9 \\
$71 \mathrm{~b}$ & $3-\mathrm{F}$ & 0 \\
$71 \mathrm{c}$ & $4-\mathrm{F}$ & 13 \\
$71 \mathrm{~d}$ & $3-\mathrm{Cl}$ & 50 \\
$71 \mathrm{e}$ & $4-\mathrm{Cl}$ & 12 \\
$71 \mathrm{f}$ & $3,4-\mathrm{Cl}_{2}$ & 34 \\
$71 \mathrm{~g}$ & $3-\mathrm{Br}$ & 1 \\
$71 \mathrm{~h}$ & $4-\mathrm{Br}$ & 0 \\
$71 \mathrm{i}$ & $3-\mathrm{NO}_{2}$ & 43 \\
$71 \mathrm{j}$ & $4-\mathrm{NO}_{2}$ & 43 \\
Rifampicin & & $>98$ \\
\hline
\end{tabular}




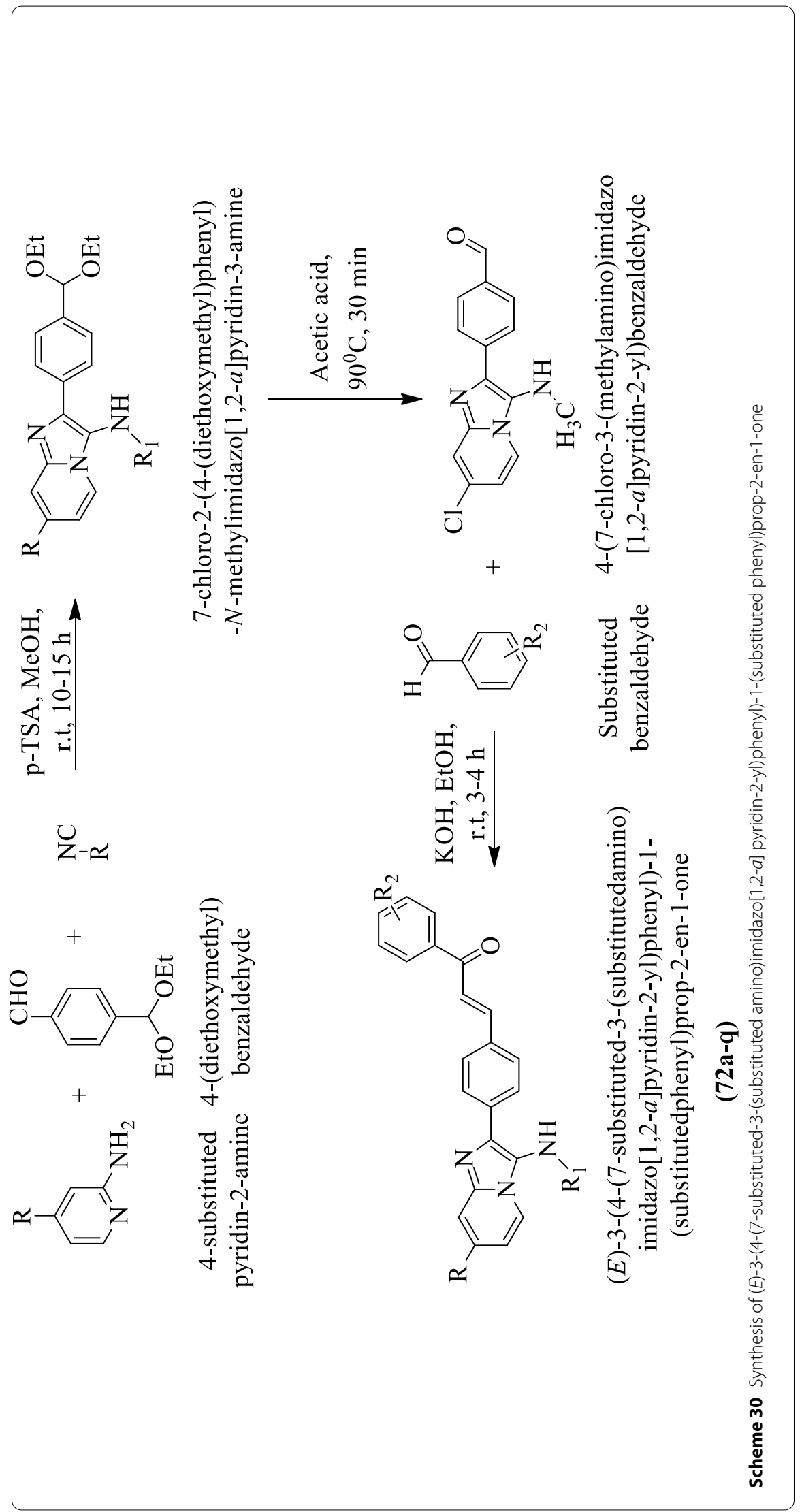


Table 30 Antitubercular activity of synthesized compounds (72a-q) against $M$. tuberculosis $\mathrm{H}_{37} R \mathbf{R}$ Pandey et al. [48]

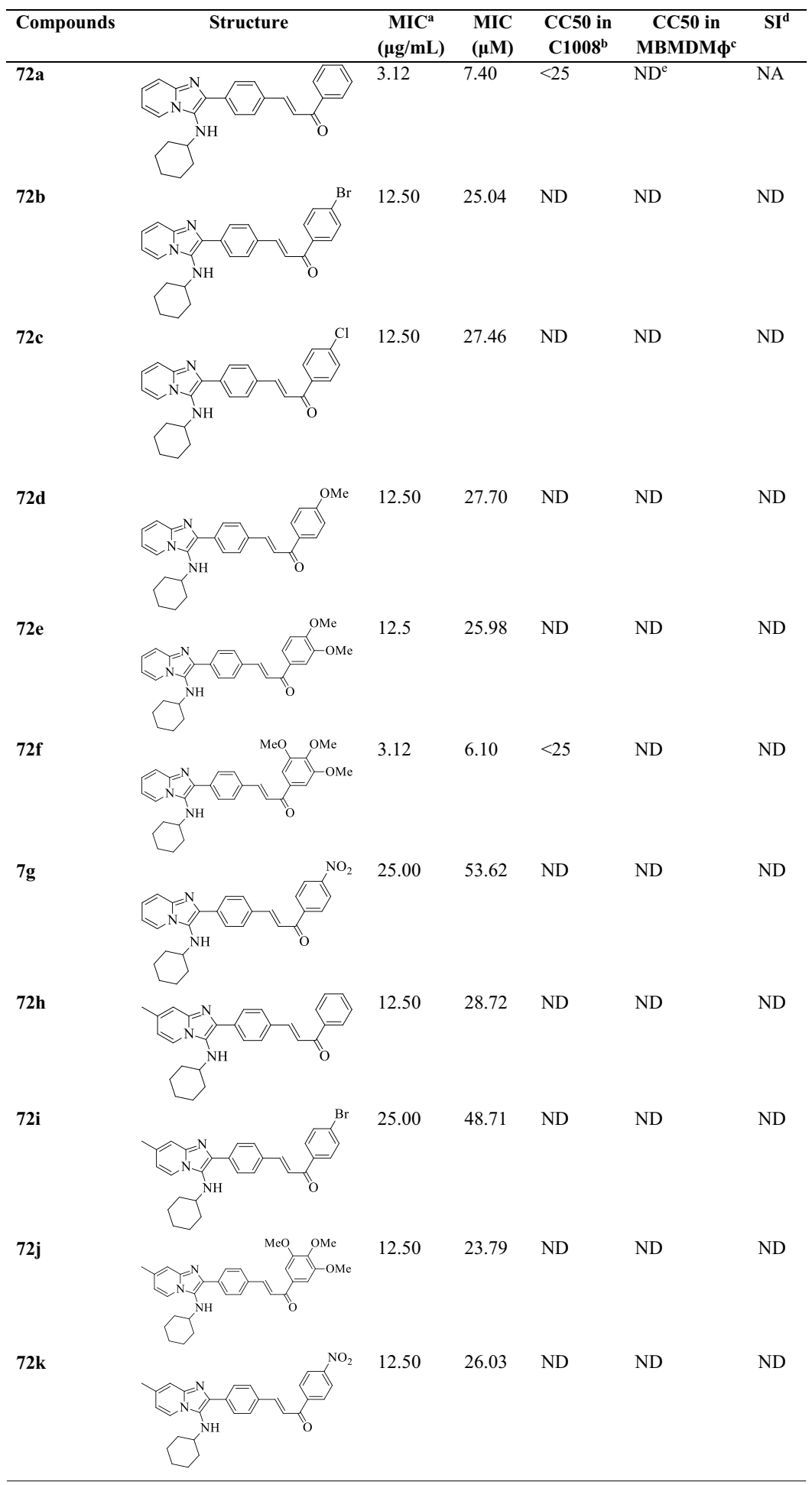


Table 30 (continued)

721

$72 \mathrm{~m}$<smiles>CC(C)(C)Nc1c(-c2ccc(/C=C/C(=O)c3ccccc3)cc2)nc2ccccn12</smiles>

3.12<smiles>Cc1ccc(C(=O)/C=C/c2ccc(-c3nc4ccccn4c3NC(C)(C)C)cc2)cc1</smiles>

$72 n$

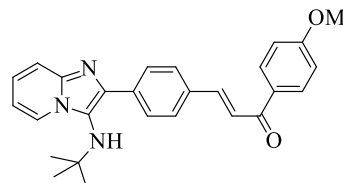

720

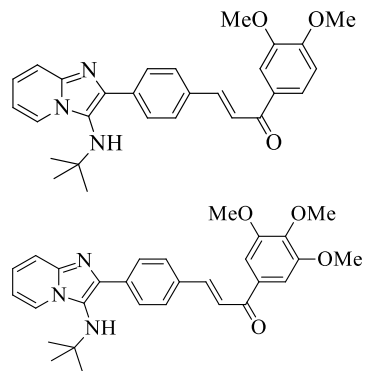

$72 q$

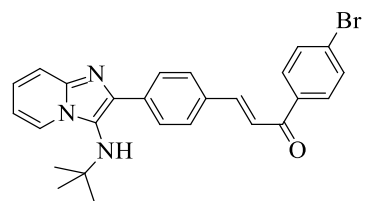

3.12

25.00

72p

25.00

7.89

$>100$

47.47

$>10$

61.09

ND

ND

ND

58.79

ND

ND

ND

6.5

$>100$

$>100$

$>10$

Ethambutol

$2.00 \quad 9.78$

Pyrazinami

$12.5 \quad 101.53$

de

${ }^{a}$ MIC: Minimum inhibitory concentration, ${ }^{b} \mathrm{C} 1008$ : vero cell lines, ${ }^{\mathrm{c}} \mathrm{MBMDM} \Phi:$ Mouse bone marrow derived macrophases, ${ }^{\mathrm{d}} \mathrm{SI}$ : Selectivity index, ${ }^{\mathrm{e}} \mathrm{ND}$ : not done. 


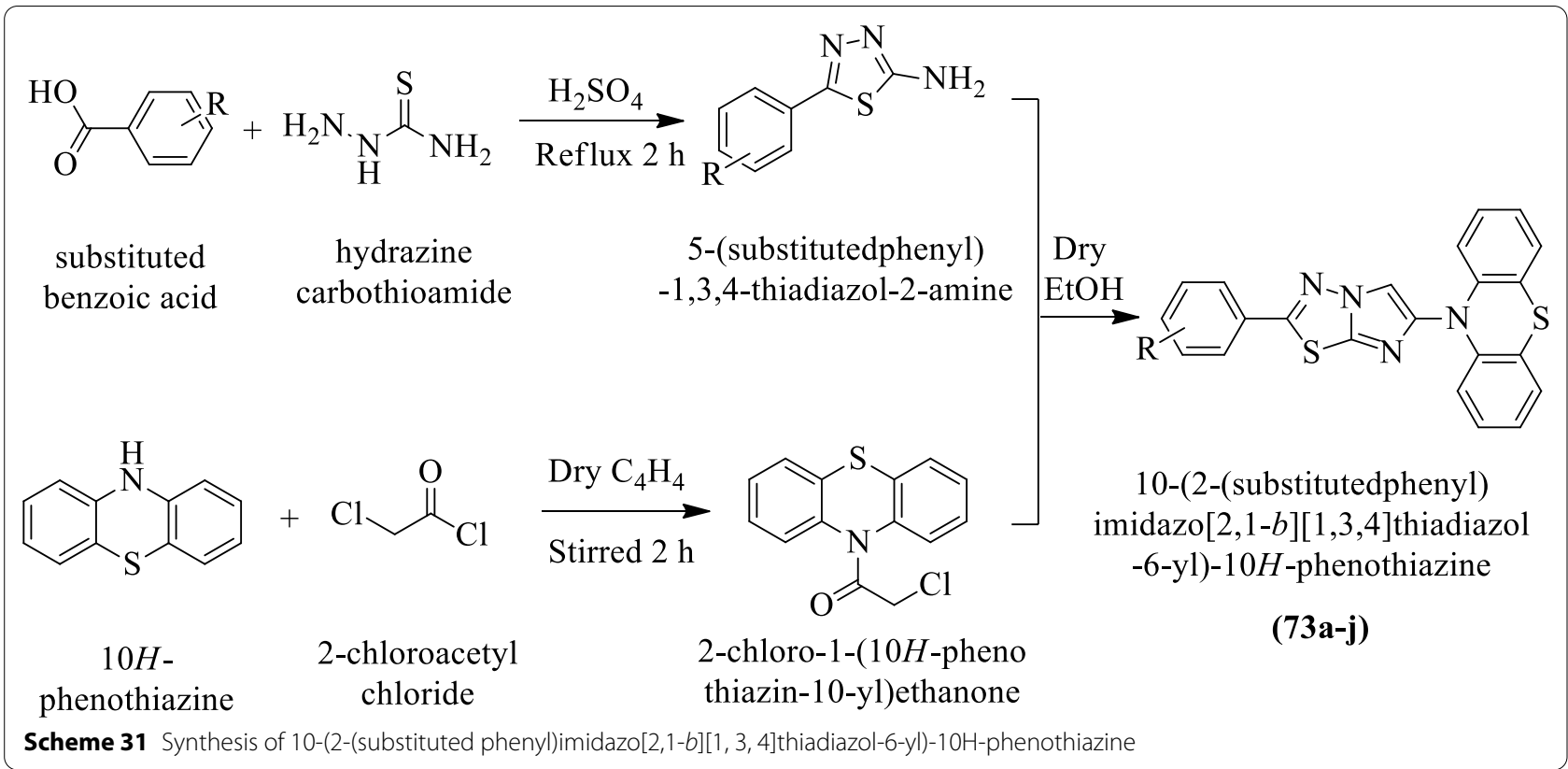

Table 31. Antitubercular activity of the synthesized compounds (73a-j) Makwane et al. [49]

\begin{tabular}{|c|c|c|c|c|}
\hline \multirow[t]{2}{*}{ Compounds } & \multirow[t]{2}{*}{ Ar1 } & \multicolumn{2}{|c|}{$\begin{array}{l}\text { Antitubercular activity inhibition (\%) (ppm) M. } \\
\text { tuberculosis H37Rv strain }\end{array}$} & \multirow[t]{2}{*}{$\begin{array}{l}\text { Antitubercular activity MIC* }(\mu \mathrm{g} / \\
\mathrm{mL}) \text { M. tuberculosis H37Rv strain }\end{array}$} \\
\hline & & 25 & 50 & \\
\hline $73 a$ & $\mathrm{C}_{6} \mathrm{H}_{5}$ & 22 & 45 & 12 \\
\hline $73 b$ & $2-\mathrm{ClC}_{6} \mathrm{H}_{4}$ & 32 & 79 & 7.5 \\
\hline $73 c$ & $3-\mathrm{ClC}_{6} \mathrm{H}_{4}$ & 36 & 80 & 6.5 \\
\hline $73 d$ & $4-\mathrm{ClC}_{6} \mathrm{H}_{4}$ & 32 & 78 & 7 \\
\hline $73 e$ & $2-\mathrm{BrC}_{6} \mathrm{H}_{4}$ & 29 & 73 & 10 \\
\hline $73 f$ & $3-\mathrm{BrC}_{6} \mathrm{H}_{4}$ & 30 & 76 & 8.5 \\
\hline $73 \mathrm{~g}$ & $4-\mathrm{BrC}_{6} \mathrm{H}_{4}$ & 30 & 75 & 9 \\
\hline $73 \mathrm{~h}$ & $2-\mathrm{NO}_{2} \mathrm{C}_{6} \mathrm{H}_{4}$ & 28 & 82 & 5.5 \\
\hline $73 i$ & $3-\mathrm{NO}_{2} \mathrm{C}_{6} \mathrm{H}_{4}$ & 27 & 84 & 4 \\
\hline $73 j$ & $4-\mathrm{NO}_{2} \mathrm{C}_{6} \mathrm{H}_{4}$ & 32 & 83 & 5 \\
\hline
\end{tabular}


Siwach and Verma BMC Chemistry

(2021) $15: 12$

Page 58 of 69<smiles>[R]c1cc(N)c(N)cc1F</smiles>

4-substituted-5- 2-(1H-imidazolfluorobenzene-1,2-diamine hydrochloride

Compounds $\mathbf{R}=$

$$
\text { 74a; } \mathrm{R}=\mathrm{Cl}
$$

74b;<smiles>COc1ccccc1</smiles>

74c;<smiles>COc1ccc(Cl)cc1</smiles><smiles>[R]c1cc2[nH]c(Cn3ccnc3)nc2cc1F</smiles>

2-((1H-imidazol-1-yl)methyl) -6-substituted-5-fluoro-1 $H$-benzo[d] imidazole (74a-e)

74d;<smiles>COc1ccc(C)cc1</smiles><smiles>Cn1c[14c][14c]([14CH3])n1</smiles>

Scheme 32 Synthesis of 2-((1H-imidazol-1-yl)methyl)-6-substituted-5-fluoro-1H-benzo[d]imidazole

Table 32 Antitubercular activity of synthesized derivatives (74a-e) against $M$. tuberculosis $\mathrm{H}_{37} \mathrm{Rv}$ strain Nandha et al. [23]

\begin{tabular}{ll}
\hline Compounds & $\begin{array}{l}\text { MIC }(\mu \mathrm{g} / \\
\mathrm{mL}) \\
\mathrm{MABA}\end{array}$ \\
\hline $74 \mathrm{a}$ & 100 \\
$74 \mathrm{~b}$ & 50 \\
$74 \mathrm{c}$ & 25 \\
$74 d$ & 50 \\
$74 \mathrm{e}$ & 12.5 \\
Isoniazid & 0.78 \\
\hline
\end{tabular}

MIC Minimum inhibitory concentration, MABA Microplate Alamar Blue Assay (visual) 
<smiles>[R]c1cc(N)c([X])cc1N</smiles>

5-substituted-4-fluoro-

2-substitutedaniline

75: $\mathbf{X}=\mathrm{NO}_{2}$

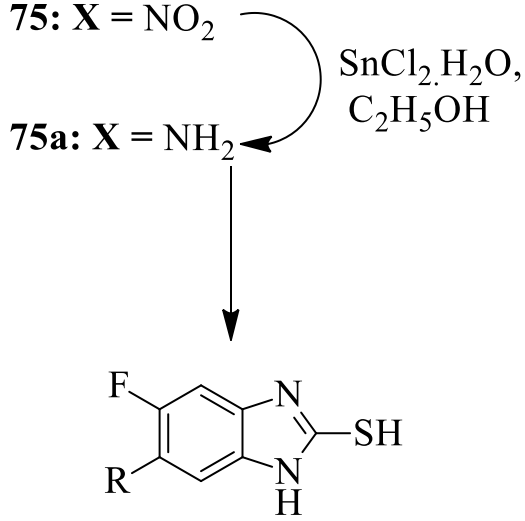

6-substituted-5-fluoro- $1 \mathrm{H}$ benzo $[d]$ imidazole-2-thiol

(76b-j)<smiles>BrCc1cc2c(cc1Br)OCO2</smiles>

5-bromo-6-(bromomethyl) benzo $[d][1,3]$ dioxole 5-substituted-4-fluoro-

2-substitutedaniline 5-(benzo[ $d][1,3]$ dioxol-5-yloxy)-2substituted-4-fluoroaniline

76: $\mathbf{X}=\mathrm{NO}_{2}$

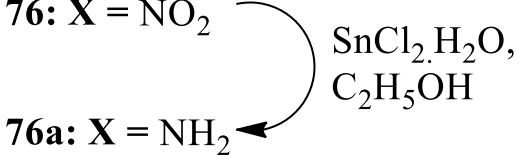
$\mathrm{Na}_{2} \mathrm{~S}_{2} \mathrm{O}_{5}$<smiles>Fc1cc2nc(Cl)[nH]c2cc1Oc1ccc2c(c1)OCO2</smiles>

6-(benzo $[d][1,3]$ dioxol-5-yloxy)-2-substituted -5-fluoro-1 $H$-benzo $[d]$ imidazole

(77a-f)

DMF, $\mathrm{K}_{2} \mathrm{CO}_{3}$, r.t, 4-6 h<smiles>[R]c1cc2[nH]c(SCc3cc4c(cc3Br)OCO4)nc2cc1F</smiles>

2-(((6-bromobenzo[ $[d][1,3]$ dioxol-5-yl) methyl)thio)-6-substituted-5-fluoro-

Scheme 33 Synthesis of 6-(benzo[d][1,3]dioxol-5-yloxy)-2-substituted-5-fluoro-1H-benzo[d] imidazole and 2-((6-bromobenzo[d][1,3]dioxol-5-yl) methyl)thio)-6-substituted-5-fluoro-1 h-benzo[d]imidazole 
Table 33 Antitubercular activity of synthesized derivatives (77a-f) and (78a-i) Nandha et al. [50]

\section{Compounds}

$77 \mathbf{a}$

77b

77c

77d

$77 e$

77f

$78 a$

$78 b$

$78 c$

78d

$78 \mathrm{e}$

$78 f$

$78 \mathrm{~g}$

$78 h$

$78 \mathbf{i}$<smiles>COc1ccc(-n2ccnc2)cc1</smiles><smiles>Cc1cc2c(cc1[N+](=O)[O-])OCO2</smiles>

$\operatorname{MIC}(\mu \mathrm{g} / \mathrm{mL}) \mathrm{MABA}$

\section{0}

50

25

50

50

100

50

50

Streptomycin

Pyrazinamide

3.12

3.12

Ciprofloxacin

MIC: Minimum inhibitory concentration, MABA: Microplate Alamar Blue Assay (visual). 


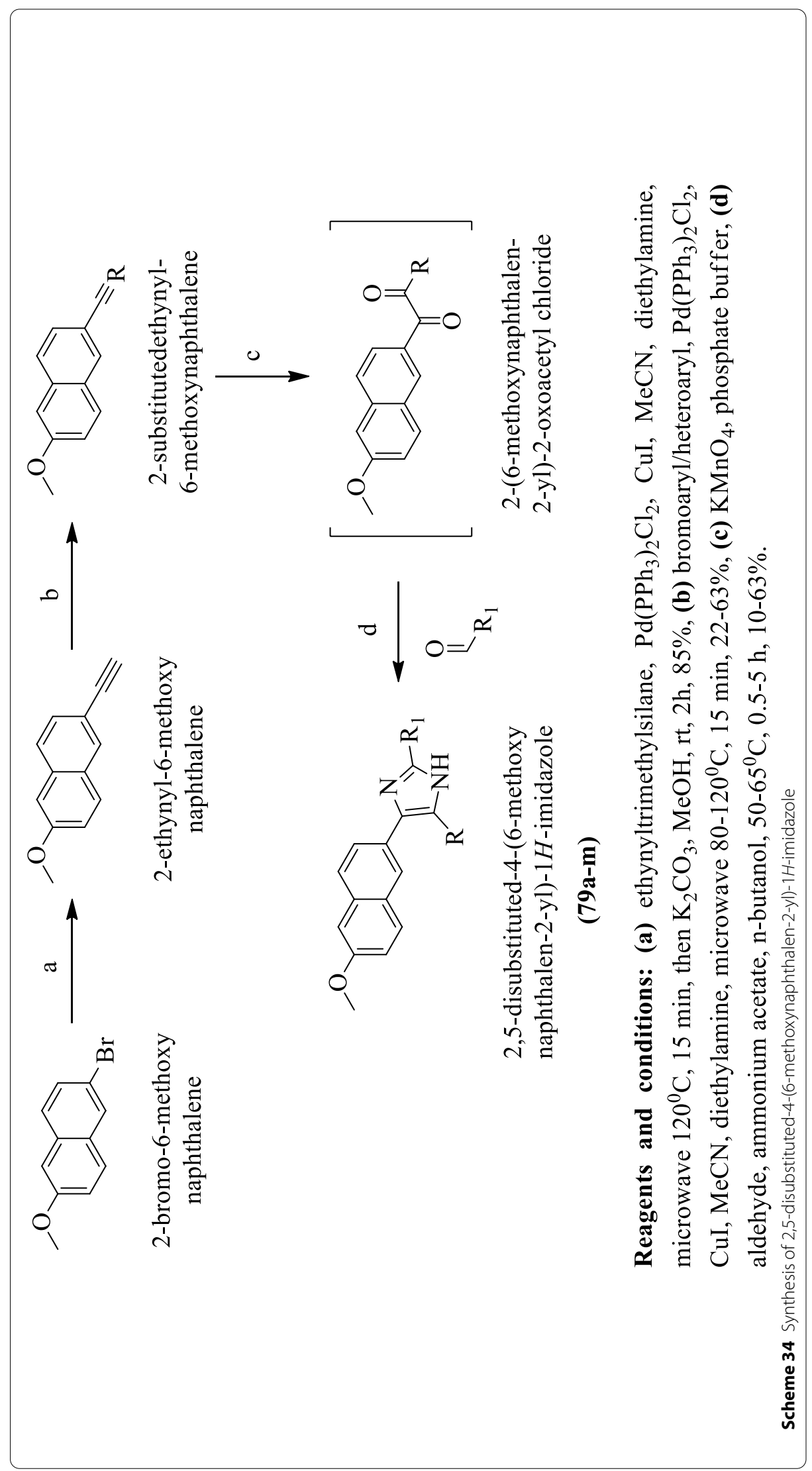


Table 34 Antitubercular activity of synthesized derivatives (79a-m) Gising et al. [51]

\begin{tabular}{|c|c|c|c|}
\hline Compounds & R5 & $\mathbf{R 2}$ & $I_{50}(\mu M)$ \\
\hline $79 a$ & & & $3.1 \pm 0.1$ \\
\hline $79 b$ & & & $>25$ \\
\hline $79 c$ & & & $>25$ \\
\hline 79d & & & $>25$ \\
\hline $79 e$ & & & $2.2 \pm 0.3$ \\
\hline $79 f$ & & & $>25$ \\
\hline $79 \mathrm{~g}$ & & & $>25$ \\
\hline $79 h$ & & & $>25$ \\
\hline $79 \mathbf{i}$ & & & $>25$ \\
\hline $79 j$ & & & $>25$ \\
\hline $79 k$ & & & $>25$ \\
\hline 791 & & & $>25$ \\
\hline $79 m$ & & & $>25$ \\
\hline
\end{tabular}


<smiles>[R]c1ccc(C(=O)OCBr)cc1</smiles>

5-(3,5-dimethyl-1 $H$-pyrazol1-yl)-1,3,4-thiadiazol-2-amine bromomethyl

4-substitutedbenzoate

a<smiles>[R]c1ccc(-c2cn3nc(-n4nc(C)cc4C)sc3n2)cc1</smiles>

6-(4-substitutedphenyl)-2-(3,5-dimethyl-1H -pyrazol-1-yl)imidazo[2,1-b][1,3,4]thiadiazole

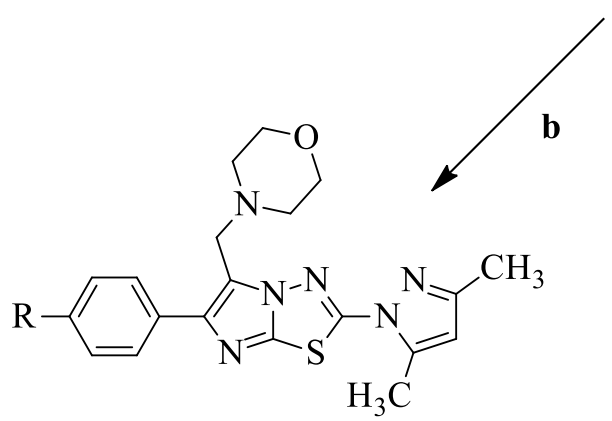

4-((6-(4-substitutedphenyl)-2-(3,5-dimethyl -1H-pyrazol-1-yl)imidazo[2,1-b] $[1,3,4]$ thiadiazol-5-yl)methyl) morpholine

(81a-e) (80a-f)<smiles>[Y]C1CCCCC1</smiles><smiles>[R]c1ccc(-c2nc3sc(-n4nc(C)cc4C)nn3c2CN2CCCC2)cc1</smiles>

6-(4-substitutedphenyl)-2-(3,5dimethyl-1 $H$-pyrazol-1-yl)-5(piperidin-1-ylmethyl)imidazo [2,1b] $[1,3,4]$ thiadiazole

6-(4-substitutedphenyl)-2-(3,5-dimethyl- $1 H$ pyrazol-1-yl)-5-(pyrrolidin-1-ylmethyl) imidazo[2,1-b][1,3,4]thiadiazole

$\mathrm{R}=\mathrm{H}, \mathrm{Cl}, \mathrm{Br}, \mathrm{OCH}_{3}, \mathrm{NO}_{2}, \mathrm{CH}_{3}$

(82a-e)

Reagents and conditions: (a) dry ethanol, $\mathrm{Na}_{2} \mathrm{CO}_{3}$, reflux $12 \mathrm{~h}$, (b) Morpholine, $\mathrm{HCHO}, \mathrm{AcOH}$, Methanol, reflux $8 \mathrm{~h}$; (c) pyrrolidine, HCHOIO, AcOH, Methanol, reflux $8 \mathrm{~h}$; (d) piperidine, $\mathrm{HCHO}, \mathrm{AcOH}$, methanol, reflux. 


\begin{tabular}{lc}
$\begin{array}{l}\text { Table } 35 \text { Antitubercular } \begin{array}{c}\text { activity } \\
\text { compounds (80a-83e) Syed et al. [52] }\end{array} \\
\text { of }\end{array}$ & synthesized \\
\hline Compounds & $\begin{array}{l}\text { MIC }(\boldsymbol{\mu g} / \\
\mathbf{m L}) \\
\text { MABA }\end{array}$ \\
\hline $80 a$ & 10 \\
$80 \mathrm{~b}$ & 10 \\
$81 \mathrm{a}$ & 10 \\
$81 \mathrm{~b}$ & 25 \\
$82 \mathrm{a}$ & 10 \\
$82 \mathrm{~b}$ & 25 \\
$83 \mathrm{a}$ & 10 \\
$83 \mathrm{~b}$ & 25 \\
$83 \mathrm{c}$ & 25 \\
$83 \mathrm{e}$ & 25 \\
Streptomycin & 7.5 \\
\hline
\end{tabular}

antioxidant, etc. This review article established the fact that 1,3-diazole act as useful templates for further modification or derivatization to design more potent biologically active compounds.

\section{Abbreviations}

AMR: Antimicrobial resistance; DNA: Deoxyribonucleic acid; DMF: Dimethylformamide; TEBA: Triethyl benzyl ammonium chloride; MTT: 3-(4, 5-Dimethylthiazol-2-yl)-2,5-diphenyltetrazolium bromide; C-A4: Combretastatin-A4; SRB: Sulforhodamine B assay; DLA: Dalton's Lymphoma Ascites cell line; EAC: Ehrlich's ascites carcinoma cell lines; DPPH: 2,2-Diphenyl-1-picrylhydrazyl; FRAP: Ferric reducing ability of plasma; SHR: Spontaneously hypertensive rats; MB: Middle brook; MABA: Microplate Alamar blue assay; L.J: Lowenstein-Jensen; $I C_{50}$ : Half maximal inhibitory concentration; HeLa: Henrietta Lacks; TEA: Triethanolamine; DMSO: Dimethyl sulphoxide; MIC: Minimum inhibitory concentration; TBAB: Tetrabutylammonium bromide; NCFT: National Centre of Fungal Taxonomy; MLC: Minimum Lethal concentration; p-TSA: P-Toluenesulfonic acid; MW: Microwave; CAN: Cerric ammonium nitrate; (4-SB) T (4-SPh)PHSO4: (4-Sulfobutyl)tris(4-sulfophenyl) phosphonium hydrogen sulfate.<smiles>Cn1ccnc1</smiles>

1-methyl$1 H$-imidazole

Compounds $\mathbf{R}=$

$\mathbf{8 4 a}=3$-Nitro

$\mathbf{8 4 b}=4$-Bromo

$84 \mathrm{c}=4$-Chloro

84d $=4$-Fluoro

$84 \mathrm{e}=2$-Hydroxy

84f $=4$-Nitro

84g = 4-Methyl

$\mathbf{8 4 h}=3$-Hydroxy

$\mathbf{8 4 i}=2,4$-Dichloro

$\mathbf{8 4 j}=2,4-$ Dihydoxy<smiles>Cn1ccnc1C#N</smiles>

1-methyl-1Himidazole-2carbonitrile

\section{2-bromo-1-(substituted} phenyl)ethanone<smiles>Cn1ccnc1-c1nnc(N)s1</smiles>

5-(1-methyl- $1 H$-imidazol2-yl)-1,3,4-thiadiazol-2-amine<smiles>[R]c1ccc(-c2cn3nc(-c4nccn4C)sc3n2)cc1</smiles>

6-(substitutedphenyl)-2-(1-methyl$1 H$-imidazol-2-yl)imidazo[2,1- $b][1,3,4]$ thiadiazole

\section{(84a-j)}

Reagents and conditions: (a) 4-N,N-Dimethylaminopyridine, DMF, Cyanogen bromide, stirred 15 h; (b) thiosemicarbazide, trifluoroacetic acid, reflux 15 h; (c) refluxed in dry ethanol for $18 \mathrm{~h}$.

Scheme 36 Synthesis of 6-(substituted phenyl)-2-(1-methyl-1H-imidazol-2-yl) imidazo [2,1-b] [1,3,4] thiadiazole 


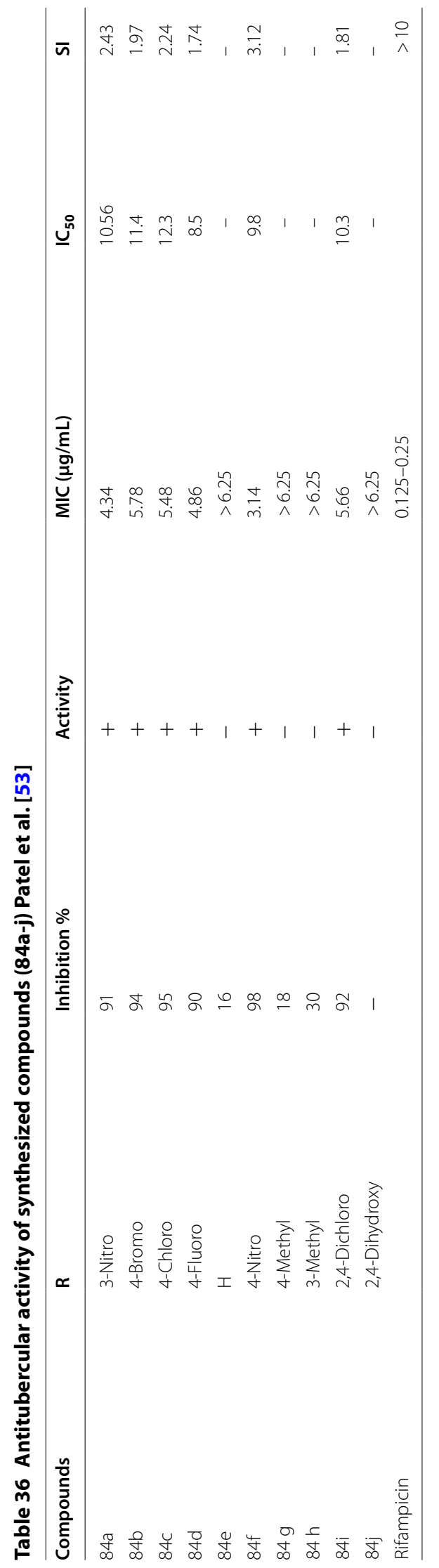




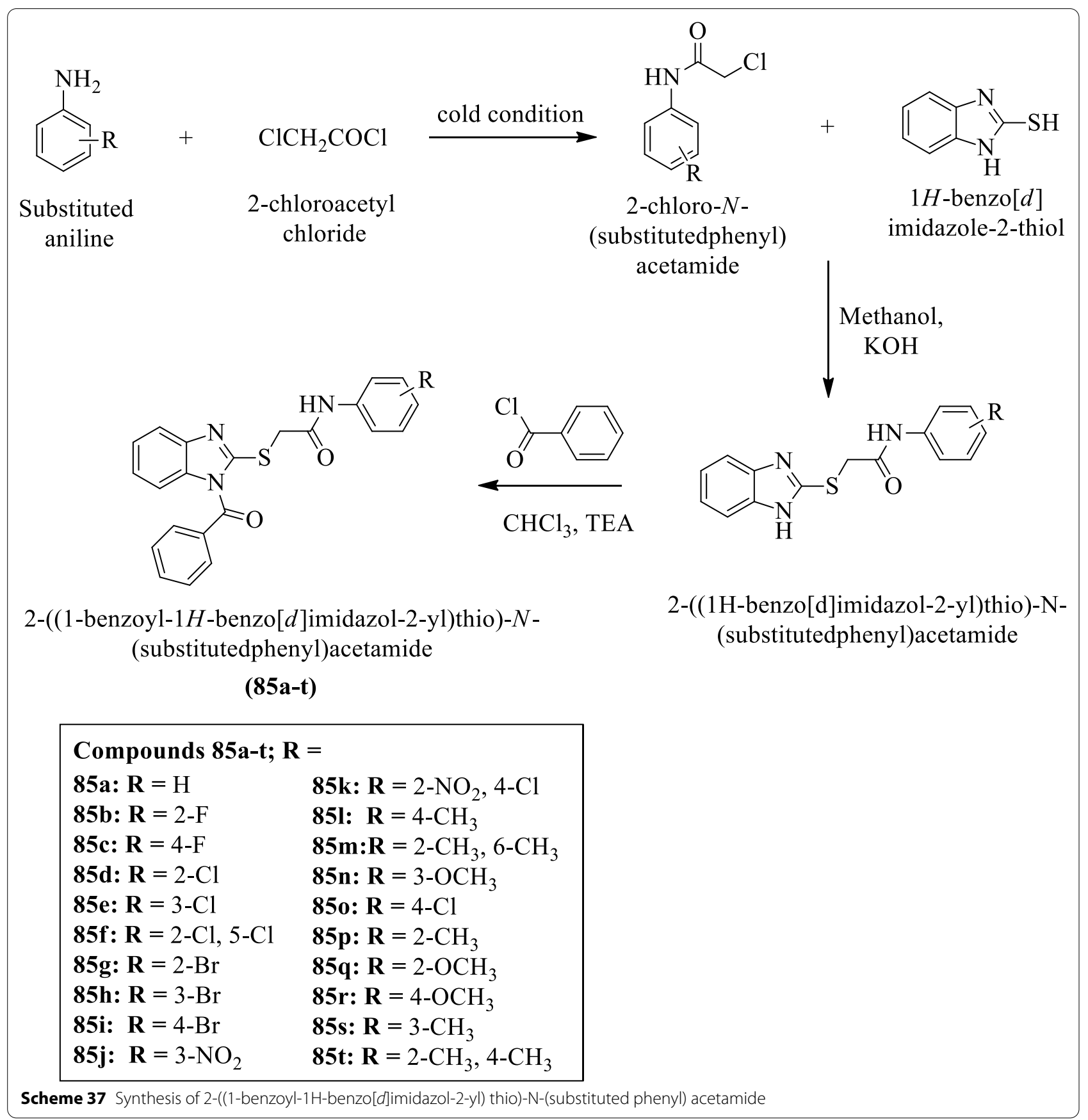


Table 37 Antitubercular activity of synthesized compounds (85a-t) Yadav et al. [54]

\begin{tabular}{|c|c|c|c|}
\hline Compounds & $\begin{array}{l}\text { Diameter of zone of inhibition }(\mathrm{mm}) \text { against H37Rv } \\
\text { (NCFT/TB/537) }\end{array}$ & MIC $(\mu \mathrm{g} / \mathrm{mL})$ & $\mathrm{MLC}(\mu \mathrm{g} / \mathrm{mL})$ \\
\hline $85 a$ & $>20$ & 12.5 & 25 \\
\hline $85 b$ & $>20$ & 12.5 & 25 \\
\hline $85 c$ & $>20$ & 12.5 & 25 \\
\hline $85 d$ & $>20$ & 12.5 & 25 \\
\hline $85 e$ & 08 & 17.8 & 28.12 \\
\hline $85 f$ & $>20$ & 12.5 & 25 \\
\hline $85 \mathrm{~g}$ & 10 & 15 & 28 \\
\hline $85 \mathrm{~h}$ & $>20$ & 12.5 & 25 \\
\hline $85 i$ & 08 & 17.8 & 28.12 \\
\hline $85 j$ & 20 & 12.5 & 25 \\
\hline $85 k$ & 10 & 15 & 28 \\
\hline 851 & $>20$ & 12.5 & 25 \\
\hline $85 \mathrm{~m}$ & $>20$ & 12.5 & 25 \\
\hline $85 n$ & NA & NA & NA \\
\hline 850 & $>20$ & 12.5 & 25 \\
\hline $85 p$ & 10 & 15 & 28 \\
\hline $85 q$ & $>20$ & 12.5 & 25 \\
\hline $85 r$ & $>20$ & 12.5 & 25 \\
\hline $85 \mathrm{~s}$ & NA & NA & NA \\
\hline $85 t$ & 10 & 15 & 28 \\
\hline Streptomycin & $>20$ & 12.5 & 25 \\
\hline
\end{tabular}

\section{Acknowledgements}

Thanks to Head Prof. Sanju Nanda, Department of Pharmaceutical Sciences, M.D.U, Rohtak for providing library and internet facilities, etc.

\section{Authors' contributions}

PKV-endeavored and accomplished the scheme; AS-completed review work and wrote the manuscript. Both authors read and approved the final manuscript.

\section{Funding}

No funding was obtained for this study.

\section{Availability of data and materials}

All data are provided in the manuscript or cited in the references.

\section{Ethics approval and consent to participate}

Not applicable.

\section{Competing interests}

The authors have no conflicts of interest.

Received: 28 January 2020 Accepted: 15 December 2020 Published online: 18 February 2021

\section{References}

1. Narasimhan B, Sharma D, Kumar P, Yogeeswari P, Sriram D (2011) Synthesis, antimicrobial and antimycobacterial evaluation of [2-(substituted phenyl)-imidazol-1-yl]-pyridin-3-yl-methanones. J Enzyme Inhib Med Chem 26(5):720-727

2. Brahmbhatt $H$, Molnar M, Pavić $V$ (2018) Pyrazole nucleus fused trisubstituted imidazole derivatives as antioxidant and antibacterial agents. Karbala Int J Mod Sci 4(2):200-206
3. Reyes-Arellano A, Gómez-García O, Torres-Jaramillo J (2016) Synthesis of azolines and imidazoles and their use in drug design. Med Chem (Los Angeles) 6:561-570

4. Verma A, Joshi S, Singh D (2013) Imidazole: having versatile biological activities. New J Chem. https://doi.org/10.1155/2013/329412

5. Bhatnagar A, Sharma PK, Kumar N (2011) A review on "Imidazoles": Their chemistry and pharmacological potentials. Int J Pharm Tech Res 3(1):268-282

6. Gueiffier A, Mavel S, Lhassani M, Elhakmaoui A, Snoeck R, Andrei G, Chavignon O, Teulade JC, Witvrouw M, Balzarini J, De Clerca E (1998) Synthesis of imidazo [1, 2-a] pyridines as antiviral agents. J Med Chem 41(25):5108-5112

7. Kumar M, Kumar D, Raj V (2017) Studies on Imidazole and its derivatives with particular emphasis on their chemical/biological applications as bioactive molecules/intermediated to bioactive molecule. Curr Synth Syst Biol 5(01):1-10

8. Zala SP, Badmanaban R, Sen DJ, Patel CN (2012) Synthesis and biological evaluation of 2,4,5-triphenyl-1H-imidazole-1-yl derivatives. J Appl Pharm Sci 2(7):22

9. Atanasova-Stamova SY, Georgieva SF, Georgieva MB (2018) Reaction strategies for synthesis of imidazole derivatives: a review. Scr Sci Pharm 5(2):7-13

10. Gupta P, Gupta JK (2015) Synthesis of bioactive imidazoles: a review. Int J Modern Chem 7(2):60-80

11. Shrivastava TP, Patil UK, Garg S, Singh MA (2013) Diverse pharmacological significance of imidazole derivatives-a review. Res J Pharm Tech 6(1):5

12. Manocha P, Wakode DS, Kaur A, Anand K, Kumar H (2016) A review: Imidazole synthesis and its biological activities. Int J Pharm Sci Res 1(7):12-16

13. Srestha N, Banerjee J, Srivastava S (2014) A review on chemistry and biological significance of benzimidazole nucleus. IOSR J Pharm 4(12):28-41

14. Romero DH, Heredia VET, García-Barradas O, López MEM, Pavón ES (2014) Synthesis of imidazole derivatives and their biological activities. J Chem Biochem 2(2):45-83 
15. Anand K, Wakode S (2017) Development of drugs based on benzimidazole heterocycle: recent advancement and insights. J Chem Biol 5(2):350-362

16. Verma BK, Kapoor S, Kumar U, Pandey S, Arya P (2017) Synthesis of new imidazole derivatives as effective antimicrobial agents. Indian J Pharm Biol Res 5(01):1-9

17. Sharma GK, Pathak D (2010) Microwave-assisted, solvent-free and parallel synthesis of some novel substituted imidazoles of biological interest. Chem Pharm Bull 58(3):375-380

18. Bhade MW, Rajput PR (2016) Design and synthesis of some imidazole derivatives containing 4-(3, 5-dichloro-2-hydroxyphenyl) imidazole moiety as antibacterial agents. Int J Appl Pure Sci Agric 2(11):80-84

19. Salman AS, Abdel-Aziem A, Alkubbat MJ (2015) Design, synthesis of some new thio-substituted imidazole and their biological activity. Am J Org Chem 5:57-72

20. Behmaram B, Foroughifar N, Foroughifar N, Hallajian S (2017) Synthesis of some derivatives of 4-phenyl-1, 3-dihydro-2H-imidazole-2-thion using ionic liquid as catalyst and evaluation of their antimicrobial activity. Int J Chem 9(2):45-51

21. Katikireddy R, Kakkerla R, Krishna MM, Durgaiah G, Reddy YN, Satyanarayana $M$ (2019) Synthesis and biological evaluation of (E)-N'-benzylidene7-methyl-2-propyl-1H-benzo [d] imidazole-5-carbohydrazides as antioxidant, anti-inflammatory and analgesic agents. Heterocycl Commun 25(1):27-38

22. Goyal A, Singh J, Pathak DP (2013) Synthesis and pharmacological evaluation of some novel imidazole derivatives for their potential anti-hypertensive activity. J Pharm Tech Res Manag 1:69-79

23. Nandha B, Nargund LG, Nargund SL, Bhat K (2017) Design and synthesis of some novel fluorobenzimidazoles substituted with structural motifs present in physiologically active natural products for antitubercular activity. Iran J Pharm Res 16(3):929

24. El-Aal EA, Fattah HA, Osman N, Seliem I (2015) Synthesis of novel imidazole and fused imidazole derivatives as cytotoxic and antimicrobial agents: molecular docking and biological evaluation. Int J Pharm Sci 7(10):36-45

25. Hsieh CY, Ko PW, Chang YJ, Kapoor M, Liang YC, Chu HL, Lin HH, Horng JC, Hsu MH (2019) Design and synthesis of benzimidazole-chalcone derivatives as potential anticancer agents. Molecules 24(18):3259

26. Subrahmanyam RS, Ramesh P, Krishna BS, D. Swaroop S, Khan MA, Darla MM, Adeepa K, Bhaskar BV, Rajendra W and Anna VR, (2017) Synthesis and biological evaluation of some new class of chromenoimidazole derivatives as probable anti-cancer agents. Rasayan J Chem 10(4):1194-1212

27. Banothu J, Gali R, Velpula R, Bavantula R (2013) Bronsted acidic ionic liquid catalyzed an efficient and eco-friendly protocol for the synthesis of 2, 4, 5-trisubstituted-1H-imidazoles under solvent-free conditions. Arab J Chem. https://doi.org/10.1016/j.arabjc.2013.10.022

28. Jain AK, Ravichandran V, Sisodiya M, Agrawal RK (2010) Synthesis and antibacterial evaluation of 2-substituted-4,5-diphenyl-N-alkyl imidazole derivatives. Asian Pac J Trop Med 3(6):471-474

29. Parab RH, Dixit BC, Desai DJ (2011) Synthesis, characterization and antimicrobial activity of imidazole derivatives. Asian J Chem 23(6):2725

30. Ahsan I, Sharma KK (2015) Pharmacological evaluation of newly synthesized imidazole derivatives containing 2-(2-hydroxyphenyl)-4, 5-diphenyl imidazole moiety. J Pharm Innov 4(3 Part B):68

31. Desai NC, Maheta AS, Rajpara KM, Joshi WV, Vaghani HV, Satodiya HM (2014) Green synthesis of novel quinoline based imidazole derivatives and evaluation of their antimicrobial activity. J Saudi Chem Soc 18(6):963-971

32. Shobhashana PG, Prasad P, Kalola AG, Patel MP (2018) Synthesis of imidazole derivatives bearing quinoline nucleus catalyzed by can and their antimicrobial, antitubercular and molecular docking studies. Res J Life Sci Bioinform Pharm Chem Sci 4(3):175

33. Selvan S, Agalya R, Maruthamuthu SR, Ruby C, Stell P (2015) Characterization and antimicrobial activity of newly synthesized benzimidazolyl compounds with docking studies. World J Pharm Res 5(2):600-607

34. Yadav S, Narasimhan B, Lim SM, Ramasamy K, Vasudevan M Shah SAA, Selvaraj M (2017) Synthesis, characterization, biological evaluation and molecular docking studies of 2-(1H-benzo [d]
imidazol-2-ylthio)-N-(substituted 4-oxothiazolidin-3-yl) acetamides. Chem Cent J 11(1):137

35. Yurttas L, Ertas M, Ciftci GA, Temel HE, Demirayak S (2017) Novel benzothiazole based imidazole derivatives as new cytotoxic agents against glioma (C6) and liver (HepG2) cancer cell lines. ACTA Pharm Sci. https:// doi.org/10.23893/1307-2080.APS.0553

36. Roopashree R, Mohan CD, Swaroop TR, Jagadish S, Rangappa KS (2014) Synthesis, characterization, and in vivo biological evaluation of novel benzimidazoles as potential anticancer agents. Asian J Pharm Clin Res 7(5):309-313

37. Romagnoli R, Baraldi PG, Prencipe F, Oliva P, Baraldi S, Tabrizi MA, LopezCara LC, Ferla S, Brancale A, Hamel E, Ronca R (2016) Design and synthesis of potent in vitro and in vivo anticancer agents based on $1-\left(3^{\prime}, 4^{\prime}\right.$, 5'-trimethoxyphenyl)-2-aryl-1H-imidazole. Sci Rep 6:26602

38. Rajendran SS, Geetha G, Venkatanarayanan R, Santhi N (2017) Synthesis, characterization and in-vitro anticancer evaluation of novel benzo [D] imidazole derivatives. Int J Pharm Sci Res 8(7):3014-3024

39. Meenakshisundaram S, Manickam M, Pillaiyar T (2019) Exploration of imidazole and imidazopyridine dimers as anticancer agents: Design, synthesis, and structure-activity relationship study. Arch Pharm. https:// doi.org/10.1002/ardp.201900011

40. Sharma GK, Sharma NK, Pathak D (2013) Microwave irradiated synthesis of some substituted imidazole derivatives as potential antibacterial and anticancer agents. Indian J Chem 52B:266-272

41. Naureen S, Ijaz F, Munawar MA, Asif N, Chaudhry F, Ashraf M, Khan MA (2017) Synthesis of tetrasubstitutedimidazoles containing indole and their antiurease and antioxidant activities. J Chil Chem Soc 62(3):3583-3587

42. Rajasekaran S, Rao G, Chatterjee A (2012) Synthesis, anti-inflammatory and anti-oxidant activity of some substituted benzimidazole derivatives. Int J Drug Dev Res 4:303-309

43. Subramaniam R, Rao G, Pai S, Virya GK, Sodhi GS (2010) Synthesis and in vitro study of biological activity of 2, 3-substituted quinazolin-4 (3H)ones. J Chem Pharm Res 2(2):462-468

44. Subhashini NJP, Kumar EP, Gurrapu N, Yerragunta V (2019) Design and synthesis of imidazolo-1, 2, 3-triazoles hybrid compounds by microwaveassisted method: evaluation as an antioxidant and antimicrobial agents and molecular docking studies. J Mol Struct 1180:618-628

45. Navarrete-Vazquez G, Hidalgo-Figueroa S, Torres-Piedra M, Vergara-Galicia J, Rivera-Leyva JC, Estrada-Soto S, León-Rivera I, Aguilar-Guardarrama B, Rios-Gómez Y, Villalobos-Molina R, Ibarra-Barajas, (2010) Synthesis, vasorelaxant activity and antihypertensive effect of benzo [d] imidazole derivatives. Bioorg Med Chem 18(11):3985-3991

46. Hadizadeh F, Hassanabad ZF, Bamshad M, Poorsoghat H, Hassanabad MF (2005) Synthesis and antihypertensive activity of new 1, 4-dihydropyridines. Indian J Chem 44B:2343-2347

47. Amini MO, Navidpour L, Shafiee A (2008) Synthesis and antitubercular activity of new N, N-diaryl-4-(4, 5-dichloroimidazole-2-yl)-1, 4-dihydro-2, 6-dimethyl-3, 5-pyridine dicarboxamides. Daru J Pharm Sci 16(1):9-12

48. Pandey AK, Sharma R, Purohit P, Dwived R, Chaturvedi V, Chauhan P (2016) Synthesis of pyrido [1,2-a] imidazo-chalcone via 3-component Groebke-Blackburn-Bienayme reaction and their bioevaluation as potent antituberculosis agents. J Chem Biol 6(5):3350-3355

49. Makwane S, Dua R (2018) Synthesis and antitubercularacitivity of New imidazo [2,1-B][1, 3, 4]-thiadiazole-phenothiazine derivatives. Arc Org Inorg Chem Sci 3(4):391-397

50. Nandha B, Nargund LVG, Nargund SL, Kuntal H (2014) Design and synthesis of imidazolylmethyl substituted fluorobenzimidazoles for antitubercular and antifungal activity. J Chem Pharm Res 6(1):530-539

51. Gising J, Nilsson MT, Odell LR, Yahiaoui S, Lindh M, Iyer H, Sinha AM, Srinivasa BR, Larhed M, Mowbray SL, Karlén A (2012) Trisubstitutedimidazoles as Mycobacterium tuberculosis glutamine synthetase inhibitors. J Med Chem 55(6):2894-2898

52. Syed MA, Ramappa AK, Alegaon S (2013) Synthesis and evaluation of antitubercular and antifungal activity of some novel 6-(4-substituted aryl)-2-(3, 5-dimethyl-1H-pyrazol-1-yl)imidazo[2,1-b][1,3,4]-thiadiazole derivatives. Asian J Pharm Clin Res 6(3):47-51 
53. Patel HM, Noolvi MN, Sethi NS, Gadad AK, Cameotra SS (2017) Synthesis and antitubercular evaluation of imidazo[2,1-b][1,3,4]-thiadiazole derivatives. Arab J Chem 10:S996-S1002

54. Yadav S, Lim SM, Ramasamy K, Vasudevan M, Shah SAA, Mathur A, Narasimhan B (2018) Synthesis and evaluation of antimicrobial, antitubercular and anticancer activities of 2-(1-benzoyl-1H-benzo[d]imidazol2-ylthio)-N-substituted acetamides. Chem Cent J 12(1):66

\section{Publisher's Note}

Springer Nature remains neutral with regard to jurisdictional claims in published maps and institutional affiliations.
Ready to submit your research? Choose BMC and benefit from:

- fast, convenient online submission

- thorough peer review by experienced researchers in your field

- rapid publication on acceptance

- support for research data, including large and complex data types

- gold Open Access which fosters wider collaboration and increased citations

- maximum visibility for your research: over $100 \mathrm{M}$ website views per year

At BMC, research is always in progress.

Learn more biomedcentral.com/submissions 